Université du Québec à Chicoutimi

\author{
Mémoire présenté à \\ 1'Université du Québec à Chicoutimi \\ comme exigence partielle \\ de la maîtrise en informatique
}

\begin{abstract}
offerte à
l'Université du Québec à Chicoutimi en vertu d'un protocole d'entente avec l'Université du Québec à Montréal
\end{abstract}

par

\title{
PATRICE ROY
}

Un modèle hybride de reconnaissance de plans pour

les patients Alzheimer :

Dilemme entrecroisé/erroné

Décembre 2007 


\section{Bibliothèque}

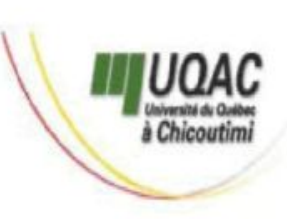

\section{Mise en garde/Advice}

Afin de rendre accessible au plus Motivated by a desire to make the grand nombre le résultat des results of its graduate students' travaux de recherche menés par ses research accessible to all, and in étudiants gradués et dans l'esprit des accordance with the rules règles qui régissent le dépôt et la governing the acceptation and diffusion des mémoires et thèses diffusion of dissertations and produits dans cette Institution, theses in this Institution, the I'Université du Québec à Université du Québec à Chicoutimi (UQAC) est fière de Chicoutimi (UQAC) is proud to rendre accessible une version make a complete version of this complète et gratuite de cette œuvre. work available at no cost to the reader.

L'auteur conserve néanmoins la The author retains ownership of the propriété du droit d'auteur qui copyright of this dissertation or protège ce mémoire ou cette thèse. thesis. Neither the dissertation or Ni le mémoire ou la thèse ni des thesis, nor substantial extracts from extraits substantiels de ceux-ci ne it, may be printed or otherwise peuvent être imprimés ou autrement reproduced without the author's reproduits sans son autorisation. permission. 


\section{RÉSUMÉ}

Les récents développements dans les technologies de l'information et l'augmentation des problèmes provenant du domaine de la santé (vieillissement de la population, pénurie de personnel médical) ont fait émerger de nouveaux axes de recherche prometteurs comme l'assistance cognitive pour les personnes atteintes de la maladie d'Alzheimer à l'intérieur d'un habitat intelligent. Une des difficultés majeures inhérentes à ce type d'assistance est la reconnaissance des activités de la vie quotidienne $(A V Q)$ réalisées par le patient à l'intérieur de l'habitat, qui sont déterminées par les actions effectuées par celui-ci, dans le but de prévoir son comportement afin d'identifier les différentes opportunités de le guider dans l'accomplissement de ses $A V Q$. Toutefois, cette situation soulève un dilemme qui n'a pas encore été pris en considération dans les travaux du domaine de la reconnaissance de plans. En effet, on peut interpréter l'observation d'une nouvelle action différente de celle attendue de deux façons opposées : soit qu'il s'agisse d'une erreur de la part du patient, soit que le patient débute une nouvelle activité, réalisée de façon entrecroisée avec l'activité en cours de réalisation.

Pour résoudre cette situation paradoxale, on propose dans ce mémoire un modèle de reconnaissance hybride comme une piste de solutions. Elle consiste en une extension probabiliste d'un modèle logique basé sur la théorie des treillis et sur un formalisme d'action en logique de description, développé durant les travaux de thèse de Bruno Bouchard effectués au laboratoire DOMUS de l'Université de Sherbrooke. Le modèle logique structure le processus de reconnaissance d'activités en un raisonnement par classification des plans possibles, permettant d'extraire un certain nombre d'hypothèses sur le comportement du patient. Notre approche mixte basée sur la logique de description probabiliste, résout le problème d'équiprobabilité en privilégiant certaines hypothèses concernant son comportement. Par conséquent, cette extension minimisera l'incertitude dans la prédiction des actions futures du patient, et plus important encore, d'anticiper les différentes catégories de déviations comportementales typiques d'un patient Alzheimer. Nous avons implémenté et validé le modèle proposé à l'aide d'un ensemble de scénarios tirés de cas réels et de fréquences d'observation inspirées d'une étude effectuée sur des patients réels. 


\section{REMERCIEMENTS}

En premier lieu, je tiens à remercier mes directeurs de recherche, $M$ Abdenour Bouzouane et M. Bruno Bouchard, pour le soutien, l'encouragement, la grande patience, la disponibilité, les commentaires et les discussions intéressantes qui ont grandement contribué à l'achèvement de ce projet.

Dans un second temps, je voudrais souligner le support irremplaçable de toute ma famille, mes parents et mon frère, qui m'ont accordé un appui et des encouragements tout le long de ce projet.

Je tiens à souligner l'appui quotidien des membres du département d'informatique et de mathématique, et plus particulièrement, je remercie M. Jean Rouette et à M. Stephen Whitney pour leur disponibilité durant mes études de maîtrise. Un merci spécial à mes collègues du groupe de recherche en informatique, spécialement à Jean-Michel Gilbert, sans qui la vie aurait été beaucoup plus monotone.

Également, je tiens à souligner le support financier du Conseil de Recherches en Sciences Naturelles et en Génie du Canada $(C R S N G)$ et de la Fondation Thomas-Léon-Tremblay.

Finalement, je tiens à remercier l'équipe du laboratoire DOMUS de l'Université de Sherbrooke et M. Sylvain Giroux pour leur collaboration. De plus, j'aimerais dire merci à tous ceux qui ont contribué à leur façon à la réalisation de ce mémoire. 


\section{TABLE DES MATIÈRES}

Résumé $\quad$ i

$\begin{array}{ll}\text { Remerciements } & \text { ii }\end{array}$

Table des matières $\quad$ v

Liste des figures $\quad$ vii

Liste des tableaux viii

$\begin{array}{ll}\text { Liste des algorithmes } & \text { ix }\end{array}$

1 Introduction $\quad 1$

1.1 Contexte . . . . . . . . . . . . . . . . . . . . 1

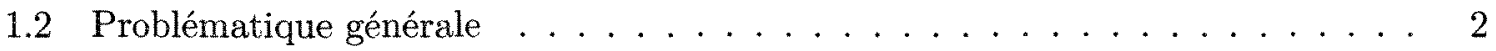

1.2.1 Aperçu de la maladie d'Alzheimer . . . . . . . . . . . . 4

1.2.2 Comportement d'un patient atteint de la maladie d'Alzheimer . . . . 5

1.2.3 La reconnaissance du comportement d'un patient . . . . . . . . . . 7

1.3 Les récents travaux sur la reconnaissance de plans . . . . . . . . . . . . 10

1.4 Apport de ce mémoire . . . . . . . . . . . . . . . . . . . . . 12

1.5 Objectifs et méthodologie de la recherche . . . . . . . . . . 15

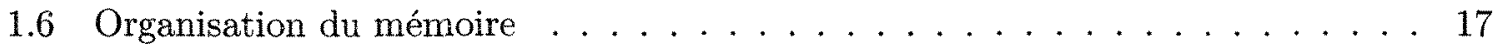


2 Les principales approches hybrides de reconnaissance de plans 19

2.1 Introduction . . . . . . . . . . . . . . . . . 19

2.1 .1 La reconnaissance de plans . . . . . . . . . . . . . . . 20

2.1.2 Les types de reconnaissance de plans . . . . . . . . . . . . . . . 22

2.1.3 Les approches de reconnaissance logiques . . . . . . . . . . . 23

2.1.4 Les approches de reconnaissance probabilistes . . . . . . . . . . 25

2.2 Les approches de reconnaissance hybrides . . . . . . . . . . . . 26

2.2.1 Les travaux de Avrahami et al. . . . . . . . . . . . . . 27

2.2 .2 Les travaux de Geib et al. . . . . . . . . . . . . . 46

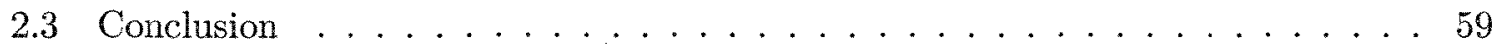

3 Un modèle hybride de reconnaissance de plans 61

3.1 Introduction . . . . . . . . . . . . . . . . . . . 61

3.2 Aperçu du modèle logique de reconnaissance de plans $\ldots \ldots \ldots$

3.2 .1 Le modèle d'action . . . . . . . . . . . . . . . . . . . 63

3.2 .2 Définition algébrique du processus de reconnaissance . . . . . . . 68

3.2.3 Exemple du processus de reconnaissance de plans . . . . . . . . . . . . 76

3.2 .4 Bilan du modèle logique . . . . . . . . . . . . . . . . 77

3.3 Le modèle hybride . . . . . . . . . . . . . . . . . . . . . . . 80

3.3.1 La logique de description probabiliste . . . . . . . . . . 80

3.3 .2 L'extension probabiliste . . . . . . . . . . . . . . . 81

3.3.3 Exemple du processus hybride de reconnaissance de plans . . . . . . . . 93

3.3.4 Bilan du processus hybride de reconnaissance de plans . . . . . . . . . 96

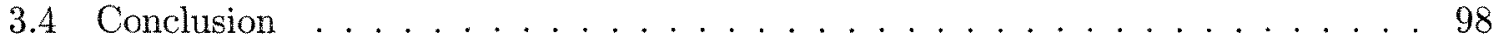

4 Validation du modèle $r$

4.1 Introduction . . . . . . . . . . . . . . . . 100

4.2 Implémentation du modèle au sein du laboratoire DOMUS . . . . . . . . . . . 101

4.2.1 L'infrastructure matérielle du laboratoire . . . . . . . . . . . . 103 
4.2.2 Architecture de la couche logicielle de bas niveau . . . . . . . . . . 105

4.2.3 Architecture du système de reconnaissance d'activités . . . . . . . . . 106

4.2.4 Outil virtuel pour la réalisation des tests . . . . . . . . . . . . 109

4.2.5 Exemple de reconnaissance d'une activité de la vie quotidienne . . . . . 110

4.3 Vérification du modèle par simulation de scénarios . . . . . . . . . . 114

4.3.1 Présentation des résultats obtenus . . . . . . . . . . . . 117

4.3 .2 Discussions sur les résultats . . . . . . . . . . . . . . 122

4.3.3 Comparaison avec les approches antérieures . . . . . . . . . . . . . 122

4.4 Conclusion . . . . . . . . . . . . . . . . . . . . 124

5 Conclusion générale $\quad 125$

5.1 Réalisation des objectifs fixés en introduction $\ldots \ldots \ldots \ldots$

5.2 Apport du mémoire . . . . . . . . . . . . . . . . . . 128

5.3 Bilan du modèle de reconnaissance proposé $\ldots$. . . . . . . . . . . . . 129

5.3 .1 Limitations de l'approche . . . . . . . . . . . . . 130

5.4 Perspectives de développements futurs . . . . . . . . . . . . 132 


\section{LISTE DES FIGURES}

2.1 Schéma illustrant de manière générale la problématique de la reconnaissance de

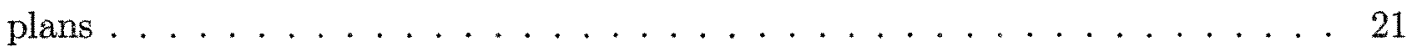

2.2 Un exemple d'une librairie de plans selon Kaminka et al. [52] . . . . . . . . 28

2.3 Un exemple d'un arbre de décision des propriétés selon Kamika et al. [52] . . 31

2.4 Propagation des marqueurs temporels dans une librairie de plans selon Kaminka

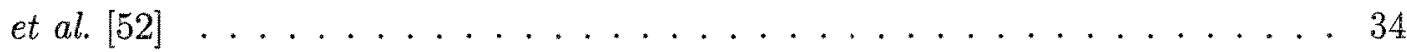

2.5 Un exemple d'un graphe d'hypothèses $G^{\prime}$ selon Kaminka et al. [52] . . . . . . . 37

2.6 Un exemple d'un arbre de décision des propriétés manquantes selon Avrahami

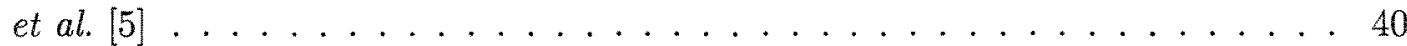

2.7 Un exemple d'une librairie de plans représentée en HHMM modifié de Avrahami

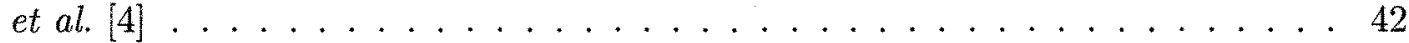

2.8 Une librairie de plans hiérarchique selon Goldman et al. [44] . . . . . . . . 46

2.9 Un réseau de croyances dynamique illustrant le modèle d'exécution de plans selon Goldman et al. [44] . . . . . . . . . . . . . . . . . . . . 49

3.1 Exemple d'un espace de reconnaissance $\Re_{o} \ldots \ldots \ldots \ldots \ldots$

3.2 Résumé du processus logique de reconnaissance . . . . . . . . . . . . . . 79

3.3 Calcul de la probabilité $P(\alpha \rightarrow \beta) \ldots \ldots \ldots \ldots$

3.4 Exemple d'un espace de reconnaissance probabilisé . . . . . . . . . . . . . 97 
4.1 Schéma général du laboratoire DOMUS $[21] \ldots \ldots$. . . . . . . . . . . 102

4.2 Schéma de l'architecture Crestron $[21] \ldots \ldots$. . . . . . . . . . . 104

4.3 Architecture de la couche de bas niveau . . . . . . . . . . . 105

4.4 Architecture de l'agent de reconnaissance de haut niveau . . . . . . . . . . 108

4.5 Un exemple de reconnaissance d'une tâche de cuisine . . . . . . . . . . 113

4.6 Les différents plans reconnus par catégories . . . . . . . . . . . . 118

4.7 Comparaison proportionnelle des types de plans anticipés . . . . . . . . . 119

4.8 Efficacité des filtres logique et probabiliste . . . . . . . . . . . . 120

4.9 Efficacité computationnelle du modèle de reconnaissance hybride en fonction du nombre d'observations. . . . . . . . . . . . . . . . . 121 


\section{LISTE DES TABLEAUX}

2.1 Probabilités d'intention des plans de l'exemple sachant les observations selon Goldman et al. $[44] \ldots \ldots \ldots \ldots \ldots \ldots \ldots$

3.1 Fréquences d'observations des plans réalisés . . . . . . . . . . 83

3.2 Fréquences de réalisations erronées et entrecroisées . . . . . . . . . 83

3.3 Fréquences de réalisation des plans de l'exemple . . . . . . . . . . . . 93

3.4 Réalisations erronées/entrecroisées de l'exemple . . . . . . . . . . . . 94 


\section{LisTE DES ALGORITHMES}

2.1 L'algorithme de requête de l'état courant selon Avrahami et al. [3] . . . . . . . 32 


\section{Chapitre 1}

\section{INTRODUCTION}

\subsection{Contexte}

Les récents développements dans les technologies de l'information et l'augmentation des problèmes provenant du domaine de la santé (vieillissement de la population, pénurie de personnel médical) ont fait émerger de nouveaux axes de recherche prometteurs comme l'assistance cognitive pour les personnes atteintes de la maladie d'Alzheimer. Actuellement, il y a 280000 Canadiens âgés de plus de 65 ans qui sont atteints de cette maladie et plus de 500000 Canadiens auront la maladie d'ici 2031 [30], d'où l'importance de ce type de recherche. Les symptômes de cette maladie entraînent chez les patients un comportement incohérent limitant leur capacité à réaliser leurs tâches de la vie courante.

Pour répondre à cette problématique, plusieurs travaux $[18,22,70,72]$ utilisent un habitat intelligent pour assister un occupant Alzheimer dans l'accomplissement de ses activités de la vie quotidienne $(A V Q)$. Cette approche est issue de l'informatique diffuse ( ubiquitous computing 》) et réfère à une tendance voulant que l'on miniaturise un ensemble de dispositifs électroniques (capteurs et effecteurs) qu'on intègre dans n'importe quel objet du quotidien, de façon transparente pour l'usager [85], dans le but de lui fournir une assistance ponctuelle. Une des difficultés majeures inhérentes à ce contexte particulier, est la reconnaissance des $A V Q$ (plans d'actions) déterminées par les actions effectuées par l'occupant et par les évènements résultants de ses actions, en vue de prédire son comportement futur et de l'assister. Cette difficulté correspond en fait à une problématique bien connue et largement étudiée dans le domaine de l'intelligence artificielle (IA), appelée la reconnaissance de plans [25]. 


\subsection{Problématique générale}

Le problème de la reconnaissance de plans ${ }^{1}$ (à l'insu ou communicative) $[1,55,77]$ réfère à une question fondamentale : comment pouvons-nous prédire le comportement d'un agent ${ }^{2}$ observé ou communiquant, de sorte que cette prédiction peut ainsi être utilisée pour des tâches de coordination, de coopération, d'assistance, ... ? La théorie de la reconnaissance de plans à l'insu [28], sur laquelle nous travaillons, tente d'établir une formalisation de cette prédiction comportementale. Elle est généralement basée sur le principe d'abduction ${ }^{3}$ pour la construction des hypothèses à propos des plans possibles, et sur un processus de correspondance faisant l'appariement entre les observations et les plans provenant d'une librairie reliée au domaine. Cette librairie décrit les plans que l'agent observé peut potentiellement exécuter. À chaque observation d'une instance d'action, l'agent de reconnaissance tente de construire les hypothèses à partir de la connaissance de la librairie. Sachant qu'il peut exister plusieurs plans possibles pouvant expliquer les mêmes observations, le défi est d'être en mesure de départager ces hypothèses concurrentes expliquant le comportement de l'agent observé.

Dans notre contexte d'assistance cognitive [22], ces prédictions sont utilisées pour identifier les différentes opportunités d'assistance qu'un habitat intelligent (agent observateur) peut offrir à un occupant atteint de la maladie d'Alzheimer à un stade précoce-intermédiaire pour accomplir ses activités de la vie quotidienne. Ce contexte augmente la complexité de la reconnaissance, de sorte que la présomption de la rationalité de l'agent observé, généralement supposée dans la littérature $[1,4,39,55]$, ne peut pas être raisonnablement maintenue. Cette supposition de la cohérence entre les intentions de l'agent observé et de ses actions est irréaliste et restreint les domaines potentiels d'application des solutions proposées dans le passé. Par conséquent, elles ne sont pas applicables à la prédiction des comportements erratiques d'un

\footnotetext{
${ }^{1}$ Cela consiste à « prendre en entrée une séquence d'actions exécutées par un acteur et d'inférer le but poursuivi par l'acteur et d'organiser la séquence d'actions en terme d'une structure de plan " [83]. Le principal objectif de cette reconnaissance est d'arriver à prévoir les actions futures de l'acteur observé.

${ }^{2}$ Une entité (humain, robot, logiciel, ... ) qui interagit avec l'environnement qu'elle habite à l'aide de capteurs et d'effecteurs $[82,87]$.

${ }^{3}$ Processus d'inférence qui permet de démarrer d'un résultat, c'est-à-dire d'un ensemble de faits observés, et de dériver, en se basant sur les règles de déductions connues, l'ensemble des prémisses ayant potentiellement provoqué ce résultat [32].
} 
patient souffrant d'Alzheimer [72]. Par exemple, une distraction ou une perte de mémoire peut amener le patient à effectuer des erreurs lors de la réalisation d'une activité. Les différents types d'erreurs peuvent êtres regroupés, en se référant à Baum et al. [14,15], en six catégories : initiation, organisation, réalisation, séquence, jugement, et complétion. Cette classification ne peut pas être seulement limitée qu'aux plans erronés, et donc, elle doit être étendue dans le but d'être placée dans un cadre de reconnaissance réactive $[4,77]$. Plus précisément, un patient à un stade précoce ne fait pas nécessairement des erreurs; il peut tout simplement arrêter temporairement l'exécution d'une activité pour en commencer une autre. De cette façon, le patient dévie de l'activité originalement planifiée en effectuant plusieurs plans entrecroisés, dans le but de réagir rationnellement à la dynamique de son environnement [79].

À partir de ce point, si on veut établir un modèle efficace de reconnaissance de plans capable de prédire n'importe quel type de comportement correct ou incorrect, la confrontation de ces deux aspects, erroné versus entrecroisé, constitue une nécessité qui est en conformité avec la réalité, mais qui est aussi paradoxale. La détection d'une nouvelle action observée, différente de celle espérée, ne peut pas être directement interprétée comme une probabilité d'interruption du plan en cours de réalisation, dans le but de poursuivre un nouveau but (l'hypothèse la plus probable). En fait, cette action peut être le résultat d'une erreur de la part d'un patient affecté mentalement ou d'un humain en bonne santé mais faillible. Toutefois, cette action inattendue n'est pas inévitablement une erreur, même dans le cas extrême, où le patient est à un stade avancé de sa maladie. Ce contexte soulève un nouveau dilemme de reconnaissance qui est principalement causé par le problème de la complétude de la librairie de plans, laquelle ne peut pas être complète dans tout domaine d'application. En ce sens, le modèle que nous présentons dans ce mémoire considère que l'agent observateur est pourvu d'une base de connaissances devant être complétée, et suppose que le patient observé effectuera potentiellement, en fonction de son déficit cognitif, des erreurs lors de la réalisation de ses activités de la vie courante (plans erronés), lesquelles ne sont pas prédéfinies dans la librairie de plans. 


\subsubsection{Aperçu de la maladie d'Alzheimer}

La maladie d'Alzheimer, ou syndrome de Démence Sénile de Type Alzheimer (DSTA), est une maladie du cerveau qui a pour caractéristique la détérioration progressive des capacités intellectuelles (pensée et mémoire) de la personne atteinte [30]. La maladie progresse lentement sur une période de sept à dix ans, pour résulter au décès du patient à l'intérieur de cette période d'évolution de la maladie. La capacité d'effectuer des activités normales (lire, conduire, cuisiner, ...) diminue graduellement, tout comme la capacité à produire des jugements et des réponses appropriées aux problèmes de tous les jours.

On peut définir le déclin cognitif de cette maladie en sept niveaux, en se référant à l'échelle globale de détérioration $(G D S)[78,79]$ des fonctions cognitives primaires d'un individu :

\section{Normal}

2. Perte de mémoire (normal pour l'âge)

3. Confusion précoce (À la frontière de la maladie d'Alzheimer)

4. Confusion tardive (Commencement de la maladie d'Alzheimer)

5. Démence précoce (Maladie d'Alzheimer à un niveau moyen)

6. Démence moyenne (Maladie d'Alzheimer à un niveau modérément sévère)

7. Démence tardive (Maladie d'Alzheimer à un niveau sévère)

Dans les premiers niveaux de dégénérescence (1-2), l'individu n'a presque pas de symptômes, et lorsque ceux-ci sont présents, il est sujet à un inconfort subjectif (le pronostic est bénin). Dans la phase terminale de la maladie (6-7), le patient souffre de symptômes sévères, lesquels entraînent des problèmes de communication verbale (aphasie), des problèmes d'identification des objets et personnes (agnosie), et des troubles élevés au niveau locomoteur (apraxie). Une personne atteinte à ce stade de la maladie d'Alzheimer doit être totalement prise en charge par une personne-ressource, car celle-ci n'est plus en mesure de subvenir seule à ses besoins fondamentaux (s'alimenter, se laver, ...).

Les niveaux intermédiaires de la maladie (3-5) constituent la phase la plus longue du processus de dégénérescence. Les principaux symptômes de ce stade de la maladie sont liés à des 
problèmes de planification causés par des pertes de mémoire sporadiques, par l'affaiblissement des fonctions exécutives, et par des problèmes de concentration diminuant l'attention sur une tâche particulière. Une distraction (un coup de téléphone, un son inhabituel, ...) ou un trouble de mémoire peuvent nuire au patient dans la réalisation de ses activités, le conduisant à réaliser les actions rattachées à une activité dans le désordre, à sauter des étapes d'une activité sans s'en apercevoir, ou à effectuer des actions qui ne présentent aucun lien avec ses objectifs initiaux [68]. À ce niveau, les personnes atteintes ne requièrent pas une prise en charge totale. Elles ont besoin d'une supervision et d'une intervention ponctuelle de la part d'un aidant. Lorsqu'on procure, sous la forme d'une assistance cognitive, un soutien continu au patient, le processus de dégénérescence de la maladie est ralenti, permettant au patient de demeurer à son domicile plus longtemps [18].

Par conséquent, le développement d'un agent artificiel ayant la capacité d'assister les patients atteints de la maladie d'Alzheimer à un stade intermédiaire lorsque la situation l'exige permettrait de diminuer la charge de travail de différentes ressources (aidant naturel, aidant professionnel, travailleur de la santé, ...). Ce type de technologie aurait le potentiel de retarder le moment fatidique d'institutionnalisation des individus atteints de la maladie, en diminuant le fardeau de tâches des personnes ressources et en redonnant une autonomie partielle aux patients. Dans la problématique de l'augmentation des coûts reliés aux services de soins à domicile, cette approche pourrait constituer une solution envisageable. Conséquemment, nos travaux de recherche s'inscrivent dans cette lignée, permettant de prendre en considération cet aspect important.

\subsubsection{Comportement d'un patient atteint de la maladie d'Alzheimer}

Lorsqu'un patient atteint de la maladie d'Alzheimer accomplit une tâche requérant une charge cognitive élevée, il est possible que des incohérences deviennent présentes dans ses agissements [72]. Dans la problématique de la reconnaissance de plans, la présence de ces incohérences se traduit par des réalisations erronées de plans en fonction de certains types d'erreurs particulières. Dans un contexte réel, les personnes ne souffrant pas de cette maladie 
peuvent aussi agir de manière incohérence, mais à un niveau beaucoup moins important et régulier qu'une personne atteinte. Contrairement aux personnes atteintes, ces personnes en bonne santé sont, généralement, en mesure de reconnaitre leurs erreurs de réalisation et d'y apporter des correctifs pour pouvoir atteindre leur but initial. On note que chez les personnes atteintes, le nombre d'incohérences augmentera de façon progressive au cours de l'évolution de la maladie, ce qui se traduira par un accroissement du nombre d'erreurs qu'ils effectuent.

En se référant à l'étude de Baum et al. $[14,15]$, on peut classifier les différents types d'erreurs de réalisation usuels d'un patient souffrant de la maladie d'Alzheimer en six catégories :

1. Initiation

\section{Organisation}

\section{Réalisation}

4. Séquence

5. Jugement

6. Complétion

Les erreurs d'initiation se produisent lorsque le patient est incapable d'amorcer une activité, même si un thérapeute lui indique qu'il doit commencer l'activité. Les erreurs d'organisation se produisent lorsque le patient est incapable d'utiliser les bons outils pour réaliser une activité. Les erreurs de réalisation se produisent lorsque le patient réalise l'activité en oubliant certaines étapes ou en ajoutant des actions qui n'ont rien à voir avec l'objectif de l'activité. Les erreurs de séquence se produisent lorsque le patient réalise de façon désordonnée les différentes étapes de l'activité en cours de réalisation. Les erreurs de jugement se produisent quand le patient réalise une activité de manière non sécuritaire et qu'il n'a pas conscience des dangers potentiels. Lorsque le patient n'est pas en mesure de déterminer si l'activité en cours de réalisation est terminée, on a alors une erreur de type complétion.

Ce système de classification permet de couvrir l'ensemble des types d'erreurs communs caractérisant le comportement déviant des patients atteints de la maladie d'Alzheimer aux différents stades de dégénérescence. Certains types d'erreurs, comme les erreurs de réalisation 
et de séquence, sont directement liés à des problèmes de planification, lesquels correspondent au comportement déviant inhérent aux individus souffrant de la maladie d'Alzheimer à un stade léger à intermédiaire [68]. Ainsi, le modèle de reconnaissance développé dans ce mémoire porte une attention plus particulière à ces deux types de réalisation erronée.

\subsubsection{La reconnaissance du comportement d'un patient}

Dans le but d'illustrer la problématique de reconnaître et prédire le comportement spécifique d'un patient atteint de la maladie d'Alzheimer à un stade intermédiaire, on utilisera un scénario inspiré d'un cas réel et tiré de l'article de Bouchard et al. [22].

Dans ce scénario, nous avons un patient dénommé Pierre, lequel est atteint de la maladie d'Alzheimer au stade 3, qui réside à l'intérieur d'un habitat intelligent. Supposons que Pierre est dans la cuisine de son appartement sur l'heure du dîner et qu'il effectue l'action de saisir une boîte de pâtes. L'agent présent dans l'appartement observe alors la réalisation de cette action, représentée par le terme SaisirPâtes. En utilisant sa base de connaissances (la librairie de plans), l'agent infère que cette action peut correspondre, dans ce cas, à une seule activité, laquelle consiste à cuire des pâtes, représentée par le terme CuirePâtes. Cette activité de la vie quotidienne est, dans notre cas, composée d'une séquence de trois étapes :

1. saisir une boîte de pâtes (SaisirPâtes),

2. faire bouillir de l'eau (FaireBouillirEau),

3. mettre les pâtes dans l'eau (MettrePâtesDansEau).

Si l'on considère que la base de connaissances de l'agent assistant est complète, comme la majorité des approches traditionnelles pour la reconnaissance de plans, et que le seul plan possiblement en cours de réalisation est CuirePâtes, alors la seule conclusion logique est que le patient effectuera ensuite les deux prochaines étapes du plan : l'action FaireBouillirEau suivit de l'action MettrePâtesDansEau. Néanmoins, sachant que Pierre souffre de la maladie d'Alzheimer, cette conclusion peut s'avérer erronée et incomplète. En effet, le patient peut avoir l'intention de réaliser l'activité CuirePâtes, mais sa maladie peut l'amener à agir de façon erronée. Par exemple, l'agent assistant pourrait observer l'exécution par Pierre de l'action 
MettrePâtesDansEau, suivie par l'action FaireBouillirEau. Cette dernière séquence d'actions s'avère incorrecte, car selon le schéma préétabli de l'activité, les pâtes doivent être mises dans l'eau seulement après que celle-ci soit en ébullition. Par conséquent, Pierre a réalisé son plan d'une manière erronée en commettant une erreur de type séquence. Il a été capable d'identifier l'ensemble des actions qu'il devait réaliser pour accomplir l'activité, mais il a été incapable de les organiser correctement dans le temps. On fait donc face ici à un problème de complétion de la base de connaissances, dans lequel le comportement du patient observé ne correspond à aucun plan inclus dans la base de connaissances de l'agent. Par conséquent, ce comportement constitue ce que nous appelons un extra-plan. Ce type de plan non préétabli correspond, dans cet exemple, à une réalisation incohérente de l'activité de la part du patient et, parce qu'il est absent de la base de connaissances, il est difficile à reconnaître et à anticiper.

Prenons en considération un second exemple, dans lequel l'agent, après avoir observé l'exécution de l'action SaisirPâtes, observe Pierre réaliser l'action FaireBouillirEau, suivie par l'action SaisirSachetThé. L'action observée SaisirSachetThé constitue, dans cet exemple, la dernière étape d'un autre plan présent à l'intérieur de la base de connaissances de l'agent assistant : PréparerThé. Cette activité, laquelle consiste à préparer une tasse de thé, est composée d'une séquence de trois étapes :

1. prendre une tasse (Prendre Tasse),

2. faire bouillir de l'eau (FaireBouillirEau),

3. saisir un sachet de thé (SaisirSachetThé).

On remarque ainsi que la dernière action observée par l'agent, SaisirSachetThé, est complètement étrangère à l'intention de Pierre initialement inférée, c'est-à-dire la réalisation du plan CuirePâtes. Le problème dans cet exemple est que Pierre a été victime d'une perte légère de mémoire, juste après la réalisation de l'action FaireBouillirEau, qui l'a fait complètement dévier de son intention originale. Suite à cette perte de mémoire, Pierre remarque qu'il se retrouve au beau milieu de la cuisine, tout en ne sachant pas pourquoi il est là. En voyant que l'eau bout et qu'il a soif, il en conclut qu'il devait être en train de se préparer une tasse de 
thé. Par conséquent, il tente de poursuivre cette activité en saisissant un sachet de thé. Cet exemple permet d'illustrer la réalisation erronée d'une activité selon le type d'erreur réalisation. Il est beaucoup plus complexe de reconnaître ce type d'erreur comparativement à l'exemple précédent. En effet, en plus de considérer uniquement les extra-plans qui correspondent à une désorganisation quelconque d'un plan de la base de connaissances, il est nécessaire de compléter dynamiquement la librairie de plans en considérant les relations d'intra-dépendances pouvant exister entre les différents plans possibles qui correspondent à l'ensemble des actions observées.

Finalement, considérons un troisième exemple, permettant d'illustrer le dilemme précédemment mentionné. Supposons que l'agent observe l'exécution de l'action SaisirPâtes par Pierre. Alors, il infère que Pierre a pour intention d'effectuer l'activité CuirePâtes. Maintenant, supposons que l'agent observe Pierre en train de réaliser l'action Prendre Tasse, laquelle est différente de l'action espérée FaireBouillirEau. Dans ce cas, l'agent peut alors tirer deux hypothèses possibles:

1. Pierre arrête temporairement l'exécution du plan initial CuirePâtes pour commencer l'exécution du plan PréparerThé. Ainsi, Pierre agit de façon rationnelle et réalise de manière entrecroisée les activités de la vie quotidienne CuirePâtes et PréparerThé.

2. Pierre agit de façon incohérente en déviant de son intention initiale CuirePâtes vers l'activité PréparerThé. Par conséquent, Pierre réalise de manière erronée l'activité CuirePâtes en effectuant une action qui n'a aucun lien avec l'activité en cours (erreur de réalisation).

L'agent fait donc face ici au dilemme cité précédemment : doit-il favoriser l'hypothèse d'une réalisation entrecroisée ou l'hypothèse d'une réalisation erronée de l'activité en cours d'exécution? Dans un contexte réel de reconnaissance, la plupart des gens peuvent réaliser plusieurs activités de façon entrecroisée ou bien de façon erronée, et par conséquent, on doit tenir compte aussi de cet aspect important de la problématique. 
Ainsi, le modèle proposé dans ce mémoire fournit un cadre formel pour structurer le processus de reconnaissance, afin de pouvoir prendre en considération l'ensemble des aspects dont nous venons de discuter. Le but de ce modèle est d'être en mesure de reconnaître le comportement d'un patient souffrant de la maladie d'Alzheimer et, plus spécifiquement, d'être apte à anticiper ses déviations plausibles en explicitant l'ensemble des extra-plans erronés pouvant découler de l'ensemble des observations et émaner de son comportement atypique, dans l'objectif de lui porter assistance.

\subsection{Les récents travaux sur la reconnaissance de plans}

La reconnaissance de plans a été utilisée dans plusieurs domaines de recherche [25]. En parcourant les différents travaux antérieurs dans le domaine de la reconnaissance de plans, on peut remarquer que la vaste littérature sur le sujet peut se diviser en deux voies majeures de recherche.

La première voie est composée de travaux basés sur les approches logiques $[55,86]$, lesquelles consistent à développer une théorie, en utilisant la logique du premier ordre, pour formaliser l'activité de reconnaissance en un processus de déduction ou d'abduction. En d'autres mots, ces approches cherchent à identifier, par une série de déductions logiques, l'ensemble des plans possibles, lesquels peuvent expliquer un ensemble d'actions observées. Dans ce type d'approche, on suppose que l'entité observée agit de manière rationnelle, c'est-à-dire que ses actions sont en accord avec l'ensemble de ses objectifs. Cette supposition n'est pas réaliste dans une situation réelle, plus particulièrement dans le contexte des patients atteints de la maladie d'Alzheimer, car l'entité observée peut être sujette à l'erreur lors de la réalisation d'une activité.

La deuxième voie est reliée aux modèles probabilistes $[1,17,27]$, lesquels définissent la reconnaissance de plan en termes de raisonnement probabiliste, basé principalement sur des modèles markoviens [84] ou des réseaux bayésiens [67]. L'idée principale derrière ce type d'approche est d'assigner, manuellement ou par apprentissage, une probabilité à chaque plan possible et d'utiliser un modèle stochastique qui peut mettre à jour ces probabilités en fonction 
de différents paramètres comme, par exemple, l'ensemble des nouvelles observations, l'état du système, et l'historique d'expériences du système. La principale force de ces approches est qu'elles permettent de prendre en considération le fait que certains plans sont a priori plus probables que d'autres. Néanmoins, le raisonnement probabiliste s'effectue sur l'entièreté de la librairie de plans, ce qui rend le processus de reconnaissance moins efficace si la taille de la librairie est importante. Un autre problème est que la qualité du processus de reconnaissance est directement liée à la qualité des valeurs de probabilité qui sont assignées aux différents plans. Aussi, ces approches considèrent que l'entité observée agit de manière rationnelle.

Une approche alternative naissante combine ces deux voies différentes de recherche, afin de désambiguïser plus efficacement l'ensemble des hypothèses qui peuvent expliquer l'ensemble des observations. L'intérêt de ce type d'approches est qu'elles permettent de circonscrire une portion de la librairie de plans à l'aide d'un processus symbolique (logique), avant d'effectuer un raisonnement probabiliste focalisé sur cette même portion. On distingue le modèle de Geib et al. [39], lequel est basé sur une logique probabiliste abductive dans le but de déduire un ensemble d'hypothèses quantifiées par des probabilités, de ce fait expliquant les comportements à plans multiples (entrecroisements) tout en prenant en compte de l'incertitude dans la perte d'observations. Un autre modèle, développé par Avrahami et al. [4], considère que le processus de reconnaissance de plans est le résultat d'une quantification probabiliste sur les hypothèses obtenues à partir d'un algorithme symbolique. Ce modèle exploite un système d'arbre de décision basé sur les propriétés des actions observées, lequel est similaire à celui utilisé dans le programme d'apprentissage $C 4.5^{4}$ [74], dans le but d'identifier efficacement les hypothèses possibles pour interpréter les plans simultanés. Même si ces deux approches prennent en considération la réalisation entrecroisée de différents plans, elles supposent que l'entité observée agit de manière rationnelle.

En explorant ces trois d'approches, on remarque qu'elles ne prennent pas en considération la problématique duale portant à la fois sur la reconnaissance des comportements erronés

\footnotetext{
${ }^{4}$ Le programme $C 4.5$ a pour but d'induire des règles de classification sous la forme d'arbres de décision à partir d'un ensemble donné d'exemples.
} 
et entrecroisés. Parce que ces approches supposent la rationalité de l'entité observée, toute action différente à celle espérée est le fruit d'une réalisation entrecroisée de plusieurs plans. Cette supposition n'est pas réaliste, car cette action peut être le résultat d'une réalisation erronée d'un plan. En effet, dans une situation réelle, un être humain en bonne santé peut, à l'occasion, effectuer des erreurs lorsqu'il réalise une activité de la vie quotidienne. Dans un contexte d'assistance aux patients atteints de la maladie d'Alzheimer, l'aspect de la réalisation erronée d'une activité a une importance capitale, et ne doit en aucun cas être négligé lors de la conception d'un modèle de reconnaissance de plans.

\subsection{Apport de ce mémoire}

Notre contribution suit les traces des approches hybrides de reconnaissance de plans tout en essayant de répondre au dilemme cité précédemment. Elle correspond à la définition d'une structure de treillis, en utilisant une logique de description probabiliste [47], dans le but de formaliser un espace de reconnaissance composé d'hypothèses expliquant des comportements erronés et entrecroisés. Ce modèle de reconnaissance de plans [81] peut être vu comme étant une extension probabiliste d'un modèle logique, précédemment présenté dans les travaux de Bouchard et al. [22].

L'utilisation de ce modèle logique, lequel est basé sur la théorie des treillis [29] et sur un cadre d'action formel [24] basé sur la logique de description [7], comme fondation de notre modèle hybride permet de transformer la problématique de la reconnaissance de plans en un problème de classification basé sur la relation de subsomption ${ }^{5}$ [42] provenant de la logique de description. Cette relation permet d'exploiter le paradigme de classification pour effectuer la correspondance entre un évènement observé et son type d'action qui lui correspond. Les plans possibles identifiés par ce processus de correspondance sont ensuite organisés à l'intérieur d'une structure de treillis, laquelle permet de rendre explicites les relations incertaines (partielles) entre ces plans possibles, lesquels ne sont pas forcément directement comparables.

\footnotetext{
${ }^{5}$ Elle désigne une relation hiérarchique entre des concepts, permettant de les organiser en structure taxonomique. Cette notion est proche de la relation d'héritage des langages objets, ou de la relation de sous-ensemble en logique ensembliste.
} 
Par conséquent, on peut donc générer dynamiquement un ensemble d'hypothèses concernant les comportements erronés ou entrecroisés plausibles d'un patient souffrant de la maladie d'Alzheimer, lesquels peuvent être induits par son déficit cognitif.

Néanmoins, ce modèle logique souffre d'une problématique d'équiprobabilité des hypothèses plausibles. En effet, le modèle ne peut pas privilégier certaines hypothèses parmi l'ensemble des hypothèses générées par le processus logique. Pour pallier à cette limitation, le modèle hybride proposé consiste en une extension probabiliste de ce modèle logique. Cette extension est basée sur certains aspects de différents travaux portant sur la logique de description probabiliste $[47,51,57]$. En effet, ce modèle hybride utilisera la notion de relation de subsomption partielle $[47,51]$ entre les différents plans plausibles de l'espace de reconnaissance, laquelle utilise la notion d'intervalle de probabilités [19], pour désambiguïser l'ensemble des hypothèses du modèle d'interprétation (espace de reconnaissance) généré par un processus symbolique (le modèle logique). Conséquemment, l'utilisation des probabilités au sein du modèle hybride permet de privilégier certaines hypothèses et par conséquent, permet de répondre au problème d'équiprobabilité du modèle logique (le cour du modèle hybride).

Sommairement, notre approche hybride de reconnaissance [81] consiste à trouver un modèle d'interprétation correspondant à un ensemble d'actions observées, en construisant une structure de treillis de plans (espace de reconnaissance) à partir de l'ensemble des plans possibles. Dans un premier temps, un processus symbolique permet de minimiser l'incertitude qualitative reliée aux prédictions des actions futures du patient en générant dynamiquement un ensemble d'hypothèses sur son comportement à partir du schéma d'intentions. Ce schéma d'intentions correspond à une borne inférieure de la structure de treillis et est utilisé pour extraire un ensemble d'extra-plans erronés ou entrecroisés anticipés, lesquels ne sont pas prédéfinis dans la base de connaissances (librairie de plans) de l'agent et qui peuvent être potentiellement réalisés par le patient. Ainsi, ce processus s'attaque au problème de complétion de la librairie de plans, laquelle est, dans un contexte réel, forcément incomplète peu importe le domaine. Ce processus symbolique permet de borner l'espace de reconnaissance en considérant seulement les hypothèses qui peuvent être classifiées à l'intérieur de la structure de treillis. 
Dans un deuxième temps, un processus probabiliste utilise le modèle d'interprétation minimal généré par le processus symbolique et un historique de fréquences d'observation des réalisations erronées et cohérentes des activités pour minimiser l'incertitude reliée au problème d'équiprobabilité des hypothèses. En utilisant les fréquences de réalisation des différents plans de la librairie et des différents types de plans (erreurs et entrecroisement), le processus probabilise les éléments de la structure de treillis en utilisant la relation de subsomption partielle. Par conséquent, l'incertitude sur les actions futures du patient est minimisée, car on considère seulement les hypothèses ayant les meilleures probabilités. Donc, le processus hybride procure au système d'assistance un moyen de décider le moment opportun où une action d'assistance doit être effectuée, en aidant l'habitat intelligent à identifier ses différentes opportunités d'assistance.

On peut considérer ce travail de recherche comme étant l'une des nombreuses briques nécessaires à la réalisation d'un projet de recherche de bien plus grande envergure [72] actuellement en cours au laboratoire DOMUS de l'Université de Sherbrooke ${ }^{6}$. Dans ce projet portant sur l'assistance cognitive au sein d'un habitat intelligent, une équipe multi-disciplinaire de collaborateurs composée de chercheurs en intelligence artificielle, en génie logiciel, en sciences cognitives, en psychologie, en ergothérapie, et en médecine gériatrique tentent de développer un système d'habitat intelligent ayant la capacité d'assister des patients atteints de déficiences cognitives diverses, telles que la maladie d'Alzheimer, les traumatismes crâniens et la schizophrénie, dans la réalisation de leurs activités de la vie quotidienne.

La reconnaissance d'une activité de la vie quotidienne constitue la pièce maîtresse de ce processus d'assistance [13]. Par conséquent, les travaux réalisés dans le cadre de ce projet pourront servir de fondation à de futurs projets portant sur différentes méthodes d'assistance, en fournissant un modèle d'interprétation de haut niveau du comportement du patient à l'agent assistant. De plus, les divers projets actuels et futurs du laboratoire qui portent sur la reconnaissance d'activités pourront réutiliser ce travail comme un cadre formel de base

\footnotetext{
'Le laboratoire DOMUS est financé par le Conseil de Recherches en Sciences Naturelles et en Génie du Canada (CRSNG) et par la Fondation Canadienne pour l'Innovation (FCI). Pour plus de détails sur les différents projets en cours au laboratoire, consultez le http://domus. usherbrooke.ca.
} 
qu'ils pourront étendre en y intégrant de nouveaux éléments propres à la spécificité de leur problématique, par exemple dans un contexte de reconnaissance d'un autre type de maladie. Également, il sera possible d'intégrer de nouveaux éléments au modèle, tels que des méthodes d'apprentissage, des notions de reconnaissance contextuelle, etc. Ces extensions pourront servir à raffiner les résultats produits par le système de reconnaissance et d'améliorer le modèle existant.

\subsection{Objectifs et méthodologie de la recherche}

Le projet de recherche présenté dans ce mémoire s'est déroulé en trois étapes principales, chacune comportant des objectifs bien définis.

La première phase du projet avait pour but de faire une investigation en profondeur sur la problématique présentée, en effectuant une revue de la littérature sur la problématique de la reconnaissance de plans $[25,28,53,83]$ et sur différentes approches existantes $[17,27,55]$, plus particulièrement les approches hybrides $[4,38]$. Un premier volet, consistant à une étude sur la maladie d'Alzheimer, a permis d'améliorer la compréhension sur les caractéristiques particulières du comportement des personnes atteintes. Ceci permet de mieux cerner les besoins inhérents à notre contexte applicatif spécifique, lié à la reconnaissance des activités de la vie quotidienne à l'intérieur d'un habitat intelligent dans le but d'assister un patient Alzheimer. Le second volet de cette phase de recherche consistait à réaliser un état de l'art sur les différents travaux de pointe existants. Le résultat de cette étape, présenté au Chapitre 2, permet d'entrevoir différentes pistes de solutions à explorer à partir des forces et faiblesses des approches antérieures. Par exemple, les différentes approches hybrides existantes ne tiennent pas compte du dilemme entrecroisé vs erroné. Sommairement, cette première phase nous a permis de mieux comprendre la problématique, d'évaluer différentes pistes de solutions possibles, de formuler un ensemble d'hypothèses de départ et de déterminer si celles-ci étaient bien fondées et pertinentes.

L'objectif de la seconde phase du projet consistait, à la lumière de nos investigations précédentes, à mettre de l'avant nos hypothèses de recherche, dans le but de définir un modèle 
de reconnaissance de plans qui répond aux différents besoins identifiés. L'approche adoptée consistait à améliorer le modèle logique défini dans [22], lequel est fondé sur un modèle d'action en logique de description et sur la théorie des treillis, en se basant sur la logique de description probabiliste. Ce nouveau modèle hybride a fait l'objet d'un article [81], lequel sera publié dans les actes de la conférence "The 2007 IEEE / WIC / ACM International Conference on Intelligent Agent Technology (IAT 2007) ». Cette étape a donc permis de définir les fondements théoriques pour un modèle de reconnaissance de plans hybride, lequel permet la reconnaissance et l'anticipation des comportements erronés et entrecroisés typiques des patients atteints de la maladie d'Alzheimer à un niveau léger à moyen.

La phase finale du projet avait, pour objectif, la validation du modèle hybride développé au cours de l'étape précédente, pour en vérifier sa viabilité et son opérationnalité au sein d'un contexte de simulation, lequel est basé sur des scénarios de cas réels tirés d'une étude sur les patients atteints de la maladie d'Alzheimer. À l'intérieur de cette phase, on a implémente notre modèle en ajoutant une couche probabiliste au système de reconnaissance d'activités développé par Bruno Bouchard lors de ses travaux de thèses à l'Université de Sherbrooke. Ce système de reconnaissance implémente le modèle logique définit dans les travaux de Bouchard et al. [21,22], et s'intègre à l'infrastructure du laboratoire DOMUS. Ces infrastructures consistent en un appartement standard (cuisine, salon, salle à manger, chambre et salle de bain) muni d'un ensemble de capteurs infrarouges, de tapis sensibles à la pression, de contacts électromagnétiques, de systèmes audio et vidéo, ainsi que d'étiquettes intelligentes ( Radio Frequency Identification " ou RFID) permettant d'obtenir la position et lidentification en temps réel des personnes et des principaux objets de l'environnement. Un ensemble d'outils logiciels développés au laboratoire DOMUS, dont l'application de reconnaissance $A C T I R E C$ [12], ont servi pour implémenter le modèle et pour vérifier comment il pouvait s'intégrer au sein de l'architecture existante. Une vérification du système de reconnaissance hybride développé a été effectuée en simulant un ensemble de scénarios de cas réels tirés des travaux de Baum et al. $[14,15]$, lesquels ont été effectués sur un échantillon de 106 patients souffrant, à différents stades d'évolution, de la maladie d'Alzheimer. On utilise pour cette simulation un historique 
d'observation s'inspirant des fréquences d'observations des différents types de comportements cohérents et erronés décrites dans l'étude de Giovannetti et al. [43], réalisée sur 51 patients atteints de maladies neurodégénératives (démences), dont la maladie d'Alzheimer fait partie. À partir des résultats obtenus lors de cette phase, une évaluation des performances du système sur plusieurs aspects a permis de voir différentes forces et faiblesses du modèle, ce qui nous a permis d'entrevoir différentes voies de développements futures pour l'amélioration du modèle.

\subsection{Organisation du mémoire}

Le contenu de ce mémoire est organisé en trois chapitres principaux, lesquels correspondent à chacune des étapes de réalisation du projet de recherche.

Le Chapitre 2 constitue un état de l'art portant sur les principales approches antérieures proposées pour répondre à la problématique de la reconnaissance de plans. On portera une attention plus particulière sur les approches de reconnaissance hybrides [4,38], lesquelles sont connexes au modèle proposé.

Le Chapitre 3 présentera en profondeur le modèle hybride de reconnaissance que nous avons développé. Dans un premier temps, nous donnerons les principaux détails sur le modèle d'action basé sur la logique de description [24], lequel sert de fondation à notre approche. Ensuite, on présentera les principaux éléments du modèle logique de reconnaissance [22] basé sur ce modèle d'action, lequel constitue le cour de notre approche hybride. Un exemple du processus logique de reconnaissance permettra au lecteur de mieux comprendre les différentes notions présentées. Finalement, les fondements de notre approche hybride [81] seront présentés, laquelle consiste en une extension du modèle logique précédent basé sur la logique de description probabiliste. Un exemple du processus hybride de reconnaissance permettra au lecteur de mieux percevoir comment cette extension probabiliste permet de raffiner les résultats obtenus à partir du modèle logique.

Le Chapitre 4 présentera le cas de validation et l'implémentation réalisée à partir du modèle hybride proposé. Cette partie permettra de spécifier les différents aspects techniques de cette réalisation, ainsi que l'architecture du système. Un scénario simple permettra d'illustrer 
les fonctionnalités de l'application. La dernière section de ce chapitre portera sur la simulation effectuée et sur les résultats obtenus. Cette section permettra de présenter les forces et faiblesses de l'approche proposée, ainsi que des pistes d'amélioration du modèle pour de futurs travaux. Finalement, une conclusion générale de ce mémoire permettra de faire un bilan sur notre apport à l'avancement du domaine et sur de nouvelles voies de recherches intéressantes qui pourraient en découler. À titre d'exemple, on peut envisager d'améliorer le modèle en ajoutant la prise en compte de différentes relations temporelles entre les actions. Cela permettra d'enrichir la librairie de plans et d'améliorer le processus de reconnaissance de plans en rehaussant la structure de plan, laquelle pourra décrire des plans plus complexes. 


\section{ChapitRe 2}

\section{LES PRINCIPALES APPROCHES HYBRIDES DE RECONNAISSANCE DE

\author{
PLANS
}

\subsection{Introduction}

Dans la vie de tous les jours, l'être humain infère les buts des personnes interagissant avec lui, ainsi que la façon d'atteindre ces buts. Par exemple, si une personne nous demande où se trouve le bureau de poste le plus proche et quel est le montant des frais d'expédition rapide pour les colis vers l'étranger, on peut raisonnablement conclure que cette personne veut envoyer un objet rapidement à une personne habitant un pays étranger et que la prochaine action de cette personne sera d'aller au bureau de poste. On a donc inféré les buts de cette personne et une portion du plan d'actions qui permet d'atteindre ces buts. Dans le domaine de l'intelligence artificielle, on tente de doter les agents artificiels de cette faculté de déduction en élaborant différents modèles. Cette problématique a donné naissance à un axe de recherche nommé reconnaissance de plans [25]. Un agent est défini comme étant une entité qui agit sur l'environnement qu'elle habite [87]. L'agent perçoit son environnement avec des capteurs et agit sur l'environnement à l'aide d'effecteurs [82]. Un agent peut donc être de différentes natures : un être humain, un robot, un logiciel, etc.

On peut considérer la reconnaissance de plans comme étant le problème inverse de la planification. La planification est un axe de recherche important dans le domaine de l'intelligence artificielle qui consiste en l'élaboration d'une stratégie prenant la forme d'une séquence d'actionsplans permettant de solutionner un problème donné, de façon à atteindre un objectif visé. 
En d'autres termes, en planification, le but est connu, contrairement à la reconnaissance de plans où l'objectif consiste à prédire ce but. Par conséquent, dans cette problématique de planification, l'agent doit choisir une séquence d'actions qui permet d'atteindre un ensemble de buts [82]. Pour atteindre les buts fixés, l'agent doit alors suivre un plan d'actions. La reconnaissance de plans permet donc de déduire ce plan d'actions et les buts qui lui sont rattachés. Tous les changements, perceptibles ou non, de l'environnement sont le résultat d'actions [80].

\subsubsection{La reconnaissance de plans}

Il n'existe pas de définition précise du problème de reconnaissance de plans qui soit acceptée de manière unanime dans la communauté scientifique. Voici, par exemple, la définition utilisée par Schmidt et al. [83]. Le problème de la reconnaissance de plans consiste à " prendre en entrée une séquence d'actions exécutées par un acteur et d'inférer le but poursuivi par l'acteur et d'organiser la séquence d'actions en terme d'une structure de plan » [83]. Cette structure de plan organise dans le temps un ensemble d'actions et permet à l'agent acteur d'atteindre un but particulier. Tandis que l'acteur tente d'atteindre un but à travers une structure de plan, l'observateur a pour but d'inférer le but de l'acteur. Parce que l'observateur ne connaît pas la structure de plan utilisée par l'acteur, il doit faire des hypothèses sur la composition de cette structure de plan à partir de l'observation des actions exécutées par l'acteur. Ces hypothèses peuvent représenter différents buts, et l'observateur doit donc utiliser la ou les meilleures hypothèses pour inférer les structures de plan possibles et les buts possibles qui y sont rattachés.

Dans notre contexte, qui porte sur l'assistance cognitive à l'intérieur d'un habitat intelligent, la reconnaissance de plans se caractérise par un processus d'inférence de plans possibles d'un agent acteur et est basée sur :

1. les observations perçues à travers les capteurs à la suite des interactions (actions) de l'agent observé avec l'environnement,

2. un principe d'abduction pour la construction d'hypothèses (plans possibles), 
3. une méthode d'appariement des observations avec les plans de la librairie qui décrit les activités potentiellement exécutables par l'agent observé.

La Figure 2.1 présente un schéma qui illustre de manière générale cette vision de la problématique de la reconnaissance de plans. Un agent observateur possède la capacité de percevoir les évènements qui se produisent dans l'environnement et qui résultent des actions exécutées par l'agent observé. Aussi, il n'y a pas de communication directe entre l'agent observateur et l'agent acteur, ce qui est inhérent à un contexte de reconnaissance de type «keyhole " (ou à l'insu), qui sera défini dans la prochaine section. On peut noter que l'agent observateur ne connaît pas à l'avance le plan poursuivi par l'agent observé. De plus, la librairie de plans de l'agent acteur peut ne pas être complètement connue par l'agent observateur (différence dans la connaissance du domaine). Conséquemment, les agents observateur et acteur possèdent seulement une chose en commune : la perception du même environnement où ils évoluent.

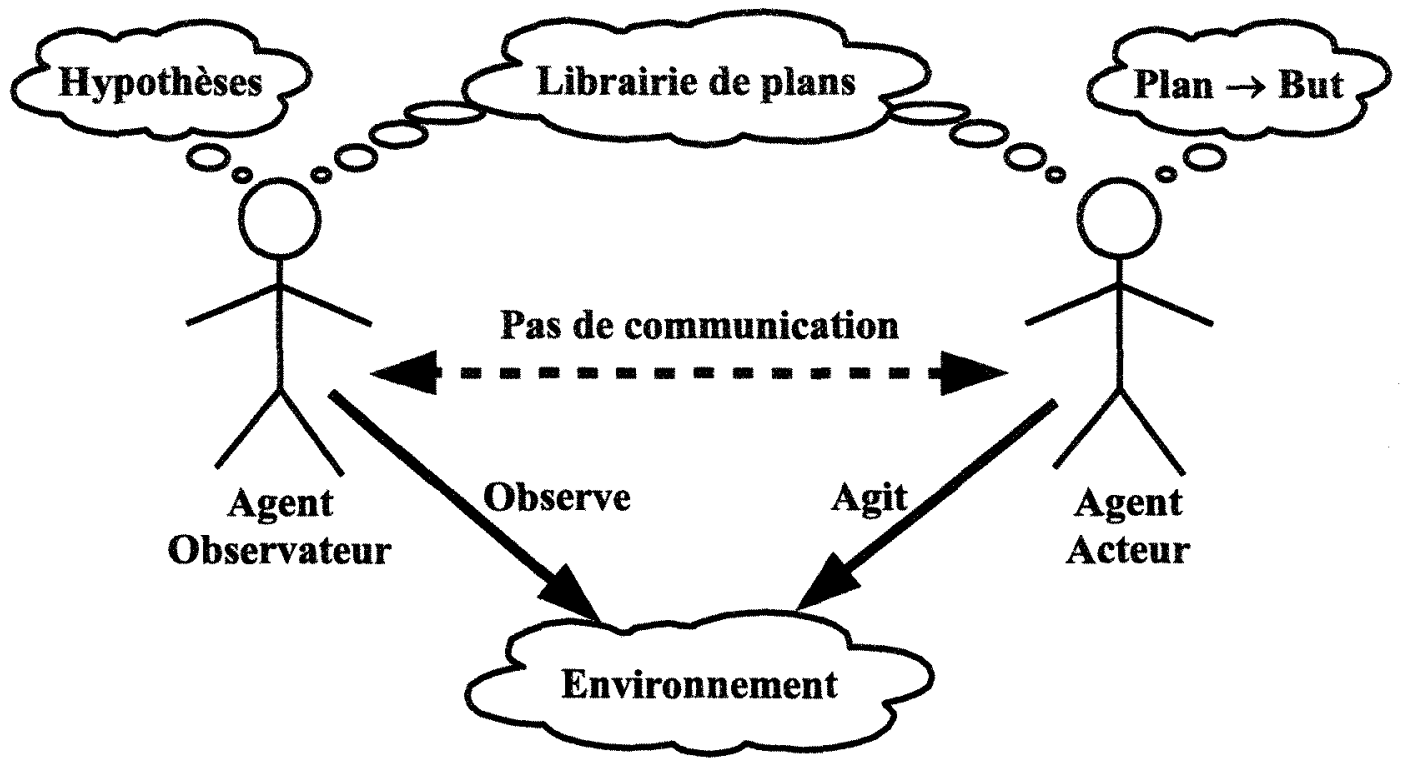

Figure 2.1 - Schéma illustrant de manière générale la problématique de la reconnaissance de plans

Dans le cadre de cette recherche, cet environnement commun correspond à l'appartement du patient observé, et la perception de cet environnement par l'agent observateur provient des capteurs dissimulés dans les différents objets de cet appartement. Le travail de l'agent 
observateur consiste, en premier lieu, à effectuer l'appariement entre les évènements perçus à l'intérieur de l'habitat intelligent et les actions de bas niveau incluses dans sa librairie. Cet appariement permet d'identifier les différentes actions exécutées par l'agent observé. On peut définir un évènement comme étant un changement perceptible d'une propriété (un fait) servant à décrire l'état de l'environnement. Donc, un évènement modifie l'état courant de l'environnement. Ces changements d'état sont produits par les actions de l'agent acteur, qui consistent en une opération volontaire exécutée par celui-ci et produisant des modifications aux propriétés des objets inclus dans l'environnement. Aussi, une action consiste à une opération de base, c'est-à-dire qu'elle ne subdivise pas en plusieurs étapes. En ce sens, un plan peut être vu comme une méta-action, c'est-à-dire une structure de haut niveau qui décrit un ensemble organisé d'actions dont la réalisation permet d'atteindre un objectif précis.

Par ailleurs, l'agent observateur doit, à chaque apparition d'une action, construire un ensemble minimal d'hypothèses de plans possibles, en se basant sur un principe d'abduction. Le raisonnement abductif est un processus d'inférence qui permet de démarrer d'un résultat, c'est-à-dire d'un ensemble de faits observés, et de dériver, en se basant sur les règles de déductions connues, l'ensemble des prémisses ayant potentiellement provoqué ce résultat [32].

L'objectif de l'agent observateur consiste, au terme du processus de reconnaissance de plans, à effectuer une prédiction la plus précise possible sur les actions futures du patient observé, dans le but d'identifier les opportunités d'assistance qui s'offriront à lui.

\subsubsection{Les types de reconnaissance de plans}

On peut scinder le problème de reconnaissance de plans en trois catégories distinctes : la reconnaissance de plans à l'insu ( «keyhole»), la reconnaissance de plans communicative (« intended 》) et la reconnaissance de plans contradictoire ( adversarial »). Dans le premier cas de figure (reconnaissance à l'insu), l'observateur suppose que l'agent observé ne tente pas d'influencer le processus de reconnaissance [25]. Dans la reconnaissance de plans communicative, l'observateur présume que l'agent observé structure délibérément ses activités afin de rendre ses intentions claires [54]. Contrairement aux deux autres approches, la reconnaissance de 
plans dite contradictoire suppose que l'agent observé est hostile à l'observation de ses actions et à l'inférence de ses plans et tente alors de nuire au processus de reconnaissance de plans [38].

Lorsqu'on parcourt les différents travaux antérieurs reliés au domaine de la reconnaissance de plans, on remarque qu'il existe deux voies de recherche distinctes : les approches de reconnaissance logiques et les approches de reconnaissance probabilistes. Afin de pallier aux différents problèmes de ces deux types d'approches, des travaux récents ont emprunté une approche dite hybride, qui combine logique et probabilité.

\subsubsection{Les approches de reconnaissance logiques}

Le premier type d'approches de reconnaissance de plans est basé sur une démarche logique $[55,86]$ qui consiste à développer une théorie utilisant la logique du premier ordre, en vue de formaliser l'activité de reconnaissance en un processus inférentiel. Cette approche a été initiée par les travaux novateurs de Kautz [54,55]. Dans les travaux de Kautz, l'agent observateur utilise une collection de schémas de plans (des types d'actions), qui sont modélisés par des axiomes en logique du premier ordre, formant une structure hiérarchique. En se basant sur cette librairie de plans, il utilise certaines règles d'inférences pour extraire un modèle d'interprétation minimale, c'est-à-dire une disjonction de plans possibles couvrant l'ensemble des actions observées. En fait, cela correspond à appliquer la théorie de la circonscription proposée par McCarthy $[62,63]$ sur la librairie de plans en se basant sur les observations. Une limite de l'approche proposée par Kautz est que le résultat du processus de reconnaissance de plans consiste en un ensemble de plans possibles équiprobables, c'est-à-dire qu'on ne peut pas privilégier une hypothèse candidate (plan possible) aux dépens des autres.

Ce problème d'équiprobabilité des plans possibles est partiellement résolu par les travaux de Wobcke [86]. Dans ces travaux, Wobcke propose une approche basée sur les travaux de Kautz qui exploite la théorie des situations [11], qui se veut un cas particulier de la théorie des mondes possibles [65]. La proposition de Wobcke consiste à encoder les plans de la librairie en termes de situations et de définir une relation d'ordre partiel qui organise ces plans par niveau de plausibilité. Cela permet d'obtenir des résultats plus raffinés en considérant, parmi l'ensemble 
des plans possibles, la classe des plans les plus plausibles. Une des faiblesses importantes de l'approche de Wobcke provient de la théorie des situations, qui est en constante évolution et dont l'appareil formel est complexe et imprécis [61]. Aussi, les plans possibles à l'intérieur d'un même niveau de plausibilité présentent encore le même problème d'équiprobabilité. En effet, même si on peut privilégier un niveau de plausibilité, on ne peut pas favoriser l'une des hypothèses à l'intérieur de ce niveau.

On peut constater, qu'en plus du problème de l'équiprobabilité des hypothèses, ce type d'approche ne considère pas les plans exécutés de manières erronées, car on suppose que l'agent observé agit de manière rationnelle. Dans notre contexte, un agent est rationnel si les actions effectuées par celui-ci sont cohérentes avec ses intentions. En d'autres termes, l'agent acteur choisit une séquence d'actions visant à maximaliser sa performance dans l'accomplissement de ses objectifs. Par conséquent, on considère que l'agent observé n'effectuera pas d'erreur lors de la réalisation d'un plan. Dès lors, l'apparition d'une action autre que celle espérée sera considérée comme étant l'interruption du plan en cours d'exécution, dans l'objectif de poursuivre un nouveau but. Cette supposition n'est pas réaliste, car cette nouvelle action peut être le résultat d'une erreur de la part d'un patient atteint de troubles cognitifs ou d'une personne en bonne santé mais faillible. On obtient donc un dilemme sur l'apparition d'une action observée qui est différente que celle espérée : exécution erronée versus exécution entrecroisée. Une exécution erronée consiste au fait que l'agent observé peut effectuer des erreurs lors de l'exécution du plan en cours. Une exécution entrecroisée consiste au fait que l'agent interrompt l'exécution du plan en cours pour suivre l'exécution d'un autre plan, pour ensuite y revenir plus tard.

Un autre problème dans cette approche est que l'on considère que la librairie de plans connus par l'agent observateur est complète, c'est-à-dire que toutes les activités possiblement exécutables par l'agent observé sont connues par l'agent observateur. Cette supposition est irréaliste dans un contexte où l'agent observé est irrationnel (peut faire des erreurs). En effet, même si l'on ajoute des façons erronées ou entrecroisées d'exécuter des plans à l'intérieur de la librairie de plans, elle sera forcément incomplète, car il peut exister une infinité de façons 
d'effectuer des erreurs lors de l'exécution d'un plan ou de combiner les différents plans lors d'une exécution de multiples plans.

\subsubsection{Les approches de reconnaissance probabilistes}

Le deuxième type d'approches de reconnaissance de plans est basé sur des modèles probabilistes $[1,17,27]$, avec ou sans apprentissage, qui s'appuient sur un modèle bayésien [67] ou markovien [50] pour définir la reconnaissance de plans en terme de raisonnement probabiliste. Ce type d'approche permet de répondre au problème d'équiprobabilité des hypothèses résultant de l'approche logique de reconnaissance. En effet, l'idée générale consiste à attribuer une probabilité à chacun des plans possibles (hypothèses) et de définir un modèle stochastique qui permet de réviser ces probabilités en fonction des actions observées et de l'état connu de l'environnement. Conséquemment, le résultat du processus de reconnaissance consiste aux plans possibles possédant la probabilité la plus élevée. On peut subdiviser cette approche en deux catégories de modèles stochastiques : les réseaux bayésiens et les modèles markoviens.

Les travaux de Charniak [27], qui sont les précurseurs de ce type d'approches, sont basés sur les réseaux bayésiens. Un réseau bayésien est défini comme étant une structure de graphe orienté sans cycle ( $D A G$ » ou «Directed Acyclic Graph»), où les nœuds représentent des variables aléatoires et les arcs représentent les dépendances informationnelles ou causales entre les variables $[71,82]$. Les dépendances sont quantifiées par des probabilités conditionnelles pour chaque nœud sachant ses parents dans le réseau. Le réseau bayésien supporte le calcul des probabilités de tout sous-ensemble de variables sachant des évidences à propos de n'importe quel autre sous-ensemble. Dans les travaux de Charniak, on se base sur un langage de premier ordre sans quantificateur pour représenter les plans et sur un système d'inférences probabilistes exploitant les réseaux bayésiens. Les variables du réseau correspondent à des propositions et les racines correspondent à des hypothèses sur le plan en cours de réalisation par l'agent observé. La probabilité a posteriori de chacune de ces hypothèses (plans possibles) est calculée en propageant les influences conditionnelles à travers le réseau, suivant un évènement observé. Par conséquent, la mise à jour des probabilités dans le réseau permet de sélectionner l'interprétation 
(l'hypothèse) la plus probable pour un ensemble d'actions observées donné. L'un des problèmes des approches bayésiennes est la complexité des calculs lors de la propagation dans le réseau, surtout remarquée lorsque la taille du réseau est relativement grande. Aussi, l'estimation des probabilités a priori est une tâche difficile et importante, car la qualité de l'inférence probabiliste dépend de la qualité de cette estimation.

D'autres travaux, comme ceux de Boger et al. [17], proposent de définir le processus de reconnaissance de plans en termes de processus de décision markovien [84]. Dans le contexte de la reconnaissance de plans, la base de connaissances de l'agent observateur est définie à l'aide d'un ensemble discret d'états qui spécifie toutes les configurations possibles de l'environnement de l'agent acteur. On peut, à l'aide d'un modèle de Markov cachées [76], caractériser la dynamique des différents états. Dans ce cadre, une probabilité de correspondance est attribuée à chacun des états, moyennant une certaine observation, ainsi qu'à chacune des transitions possibles d'un état à un autre (actions). Ainsi, le processus de reconnaissance de plans consiste à évaluer la succession d'états pouvant le mieux expliquer les actions observées, c'est-à-dire celle avec la meilleure somme de probabilités. L'avantage des approches markoviennes, par rapport aux approches bayésiennes, est de réduire la complexité reliée au calcul des probabilités.

On peut aussi noter que les approches probabilistes ne considèrent pas l'exécution entrecroisée des plans, car le processus d'inférence probabiliste, en fonction de l'ensemble des actions observées, donne comme résultat l'hypothèse (plan possible) avec la meilleure probabilité parmi les plans de la librairie de l'agent observateur.

\subsection{Les approches de reconnaissance hybrides}

Afin de pallier aux différents problèmes des approches logiques et probabilistes de reconnaissance de plans, un troisième type d'approches combinant logique et probabilité a été développé récemment : les approches de reconnaissance hybrides. Ce type d'approche hybride s'inscrit directement dans la lignée de nos travaux. Pour bien montrer en quoi consiste ce type d'approches, on présentera les deux principales approches hybrides de reconnaissance de plans développées : les travaux de Avrahami et al. et les travaux de Geib et al.. 


\subsubsection{Les travaux de Avrahami et al.}

Le modèle de reconnaissance proposé dans les travaux de Avrahami et al. $[3,4,5,52]$ utilise une quantification probabiliste sur des hypothèses obtenues à l'aide d'un algorithme symbolique. L'algorithme symbolique permet de filtrer les hypothèses valides et utilise un moteur d'inférence probabiliste pour discriminer cet ensemble d'hypothèses. Cette façon de faire permet de rendre plus efficace l'inférence probabiliste en appliquant celle-ci seulement sur un sous-ensemble d'hypothèses pré-filtrées plutôt que sur toute la librairie.

\subsubsection{Représentation de la librairie de plans}

On représente la librairie de plans de l'agent observé par un graphe orienté connexe sans cycle à une seule racine. Les sommets du graphe représentent des étapes de plan. Les arêtes du graphe peuvent être de deux types:

- les arêtes verticales qui décomposent les étapes de plan en des sous-étapes;

- les arêtes séquentielles qui spécifient l'ordre temporel attendu d'exécution.

On associe, pour chaque plan, un ensemble de conditions des propriétés observables de l'agent et de ses actions. Quand ces conditions sont présentes, on peut alors dire que les observations correspondent au plan. À n'importe quel moment, l'agent observé est censé être en train d'exécuter un chemin de décomposition de plan à partir de la racine jusqu'à une feuille particulière, à travers les arêtes de décomposition. La Figure 2.2 montre, à titre d'exemple, une partie d'une librairie de plans. On note la présence dans la figure des arêtes de décomposition (flèche pleine) et des arêtes séquentielles (flèche pointillé). Pour une représentation claire, seules les arêtes de décomposition aux premiers plans fils (dans l'ordre temporel) seront présentes sur la figure. Ainsi sur la Figure 2.2, le chemin de plan racine $\rightarrow$ défendre $\rightarrow$ tourner $\rightarrow$ avec la balle peut représenter une hypothèse sur l'état interne actuel d'un agent observé. Une collection de tels chemins de plan constituerait un ensemble d'hypothèses.

Un agent observé peut modifier son état interne de deux façons. Premièrement, il peut suivre les arêtes séquentielles, de sorte que lorsqu'il n'y a plus de liens séquentiels disponibles, il retourne au plan parent et continu à suivre les arêtes séquentielles (si elles existent). 


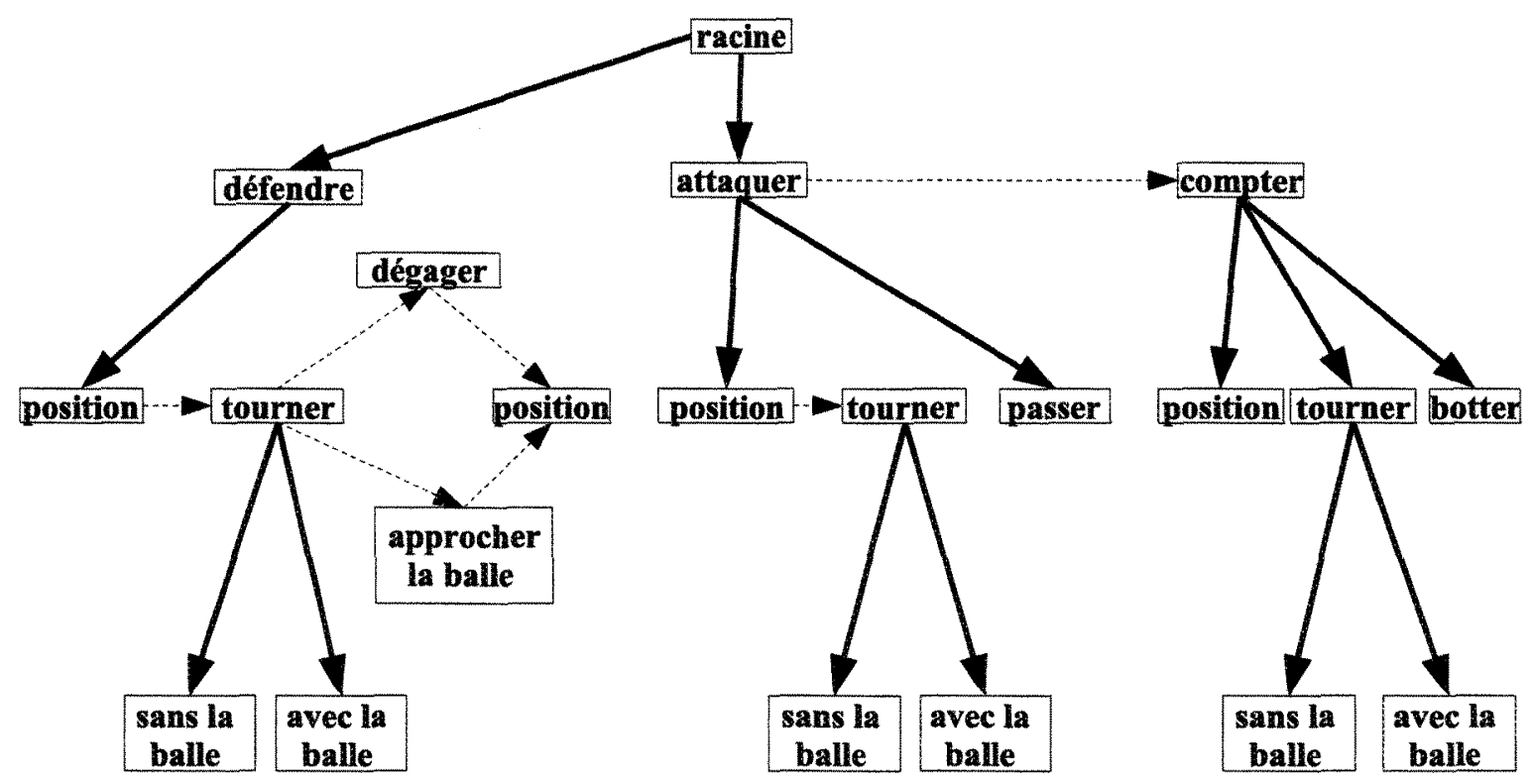

Figure 2.2 - Un exemple d'une librairie de plans selon Kaminka et al. [52]

Deuxièmement, il peut interrompre l'exécution d'un plan à tout moment pour répondre de manière réactive aux changements de l'environnement, et sélectionne un nouveau (premier) plan (il sera question plus tard du cas de l'entrecroisement, où l'agent peut reprendre une séquence de plan). Par exemple, supposons que l'agent exécute racine $\rightarrow$ défendre $\rightarrow$ tourner $\rightarrow$ avec la balle, et qu'il interrompt ce plan. Il peut maintenant choisir racine $\rightarrow$ attaquer $\rightarrow$ passer, mais pas racine $\rightarrow$ attaquer $\rightarrow$ tourner parce qu'il s'agit d'un nouveau plan et que racine $\rightarrow$ attaquer $\rightarrow$ position n'a pas été sélectionné avant. On note que la Figure 2.2 ne montre pas les conditions d'observation associées aux plans. Par exemple, on peut avoir une propriété « a la balle » où la valeur est vraie lorsqu'on observe que la balle est à proximité de l'agent observé. Le plan botter peut avoir une condition qui spécifie que a la balle = vrai, tandis que le plan approcher balle vérifierait a la balle $=$ faux.

L'algorithme de reconnaissance de plans fonctionne en trois phases incrémentales:

1. correspondance des observations (ou hypothèses d'actions) aux plans, en étiquetant ceux qui correspondent à l'aide des marqueurs temporels des actions observées ; 
2. propagation des étiquettes des marqueurs de temps à travers la librairie de plans, afin d'étiqueter des chemins de plan complets;

3. extraction des hypothèses en fonction de la requête.

Les trois prochains points présentent ces différentes phases.

\subsubsection{Correspondance des actions observées aux étapes de plans}

Les observations considérées ici sont des actions complexes qui consistent en un tuple de propriétés d'observation : l'état de l'environnement, les actions effectuées par l'agent observé, et les conditions d'exécution maintenues par l'agent. Dans un contexte réaliste, il est probable que plusieurs plans correspondent à un ensemble d'actions observées, et il y a donc plusieurs hypothèses pour l'état interne de l'agent observé. La correspondance entre les observations et les plans peut être coûteuses si l'on parcourt tous les plans de la librairie et que, pour chaque plan, on vérifie toutes les propriétés d'observations. Pour chaque plan, les conditions d'observation ne couvrent pas toutes les propriétés d'observation et il y a donc du gaspillage dans l'effort.

Pour régler ce problème, la librairie de plans est augmentée par une structure de données : l'arbre de décision des propriétés ou FDT( «Feature Decision Tree 》). Cette structure permet de faire correspondre efficacement les actions observées et les plans. Un FDT est construit de manière similaire à un arbre de décision utilisé au niveau de l'apprentissage automatique [74]. Chaque noud dans un FDT représente une propriété d'observation et chaque branche qui descend d'un noud correspond à l'une des valeurs possibles pour cette propriété. Contrairement aux arbres de décision traditionnels, chaque feuille de l'arbre pointe vers les plans qui vérifient

la propriété représentée par la feuille. De cette façon, chaque nœud dans le FDT divise un ensemble de plans en des sous-ensembles en fonction des valeurs d'une propriété. Par conséquent, la détermination des plans qui correspondent à un ensemble de propriétés d'observation est efficacement accomplie en parcourant le FDT de haut en bas et en prenant les branches qui correspondent aux valeurs observées des propriétés, jusqu'à ce qu'une feuille soit atteinte. La 
feuille pointe vers les plans qui correspondent à l'ensemble conjoint des hypothèses d'actions. Cet arbre de décision est généré avant l'exécution de l'agent de reconnaissance de plans.

Comme un arbre de décision, la construction d'un FDT peut utiliser le gain d'information (le degré de divisibilité de l'ensemble des plans en fonction de la propriété) pour déterminer les propriétés les plus importantes à vérifier en premier, pour garantir une vérification optimale des propriétés. En premier lieu, on crée un nœud racine (correspondant à tous les plans) et on lui associe les propriétés qui fournissent le gain d'information le plus élevé (divise le plus uniformément possible les plans qui vérifient la propriété). Ensuite, les nœuds fils sont créés pour chaque valeur de la propriété, et on répète récursivement le processus de sélection de la propriété qui divise le mieux les plans associés au nœud. Le processus continu jusqu'à ce que l'on ait un nœud qui pointe seulement vers un plan, ou qu'il n'y ait plus de propriétés qui différencient les plans.

Contrairement aux arbres de décision dans l'apprentissage automatique, dont la construction est basée sur des exemples de données cibles, la construction de l'arbre de décision se base sur la librairie de plans. Cette librairie de plans contient tous les plans qui peuvent être exécutés par l'agent observé. Il n'y a aucune incertitude dans la détermination de plans qui correspondent à un ensemble d'observations, et il n'est pas nécessaire d'élaguer les nœuds pour prévenir la surcharge de l'arbre. Dans le cas où certains plans ne vérifient pas une propriété, ils sont tout simplement passés dans la phase de construction à tous les nœuds fils, car ils sont consistants avec toutes les valeurs des propriétés qu'ils ne vérifient pas.

Par exemple, la Figure 2.3 montre une portion d'un FDT utilisant les propriétés associées avec les plans de la Figure 2.2, telles que la distance des autres joueurs, la possession de la balle, la visibilité du but adverse, et le numéro de l'uniforme. Le FDT sépare les plans en accord avec les valeurs de ces propriétés. Pour déterminer les plans correspondants, l'algorithme de correspondance vérifie en premier la propriété « a la balle ». En fonction de la valeur de cette propriété, l'algorithme continu dans la branche appropriée pour vérifier en séquence d'autres propriétés, jusqu'à ce qu'il atteigne un nœud feuille. Cette feuille a des pointeurs pour toutes les instances des plans qui lui sont associées dans la librairie de plan. Par exemple, la 
feuille position a quatre pointeurs dans la Figure 2.2. On peut noter que le plan tourner est applicable peu importe si « a la balle» est vraie ou fausse, et donc un nœud qui lui est associé va apparaître dans les deux sous-arbres de la racine « a la balle ».

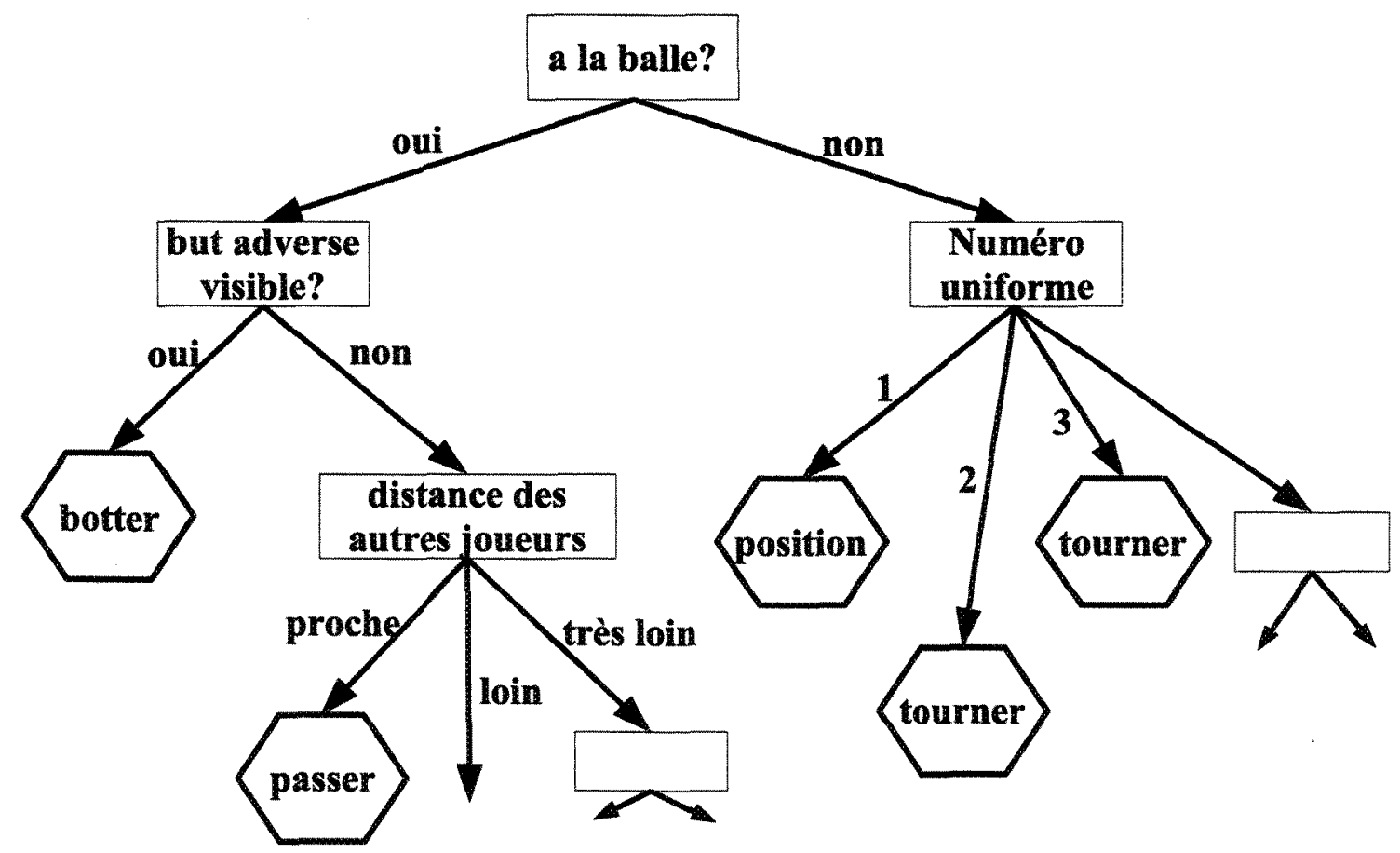

Figure 2.3 - Un exemple d'un arbre de décision des propriétés selon Kamika et al. [52]

La correspondance entre les actions observées et les plans est efficacement accomplie en utilisant le $F D T$, en suivant un chemin de la racine à une feuille. La hauteur d'un FDT est, dans le pire cas, $O(F)$ où $F$ est le nombre de propriétés. Dans ce cas, l'un des plans vérifie toutes les propriétés disponibles, ce qui n'est pas réaliste. Dans un contexte plus réaliste, la hauteur de l'arbre est plus proche du meilleur cas, qui est $O(\log F)$. Aussi, chaque feuille d'un $F D T$ pointe vers $O(l)$ plans, où $l$ est le nombre maximum de plans qui sont consistants avec une seule observation $(l \leq L)$, où $L$ représente le nombre de nœuds dans la librairie de plans. Donc, la complexité pour la correspondance des observations et des plans est d'au plus $O(F+l)$ 


\subsubsection{3 Étiquetage et Propagation}

Lorsque les plans correspondants sont trouvés, ils sont étiquetés par le marqueur temporel de l'action observée. Ces étiquettes sont propagées dans toute la librairie de plans, pour déterminer des chemins de plan complets qui constitueront les hypothèses de l'état interne de l'agent observé, au moment où les observations ont été faites.

L'une des complications de l'étiquetage est qu'un plan peut correspondre aux observations, mais peut ne pas constituer une hypothèse valide quand on considère l'historique des actions observées (inconsistance temporelle). Par exemple, si le premier ensemble d'hypothèses d'actions correspond au plan passer, et que le deuxième ensemble d'observations correspond au plan tourner. Le FDT pointe alors sur les trois instances d'actions de tourner: sous défendre, sous attaquer, et sous compter. Si l'on assume qu'il y a aucune perte d'observations, la seule instance d'actions valide de tourner est celle sous compter, car c'est la seule instance où tourner peut être sélectionnée sans passer par l'action position.

L'algorithme de requête de l'état courant 2.1 ou CSQ( « Current State Query ») permet de déterminer les hypothèses sur l'état courant de l'agent observé.

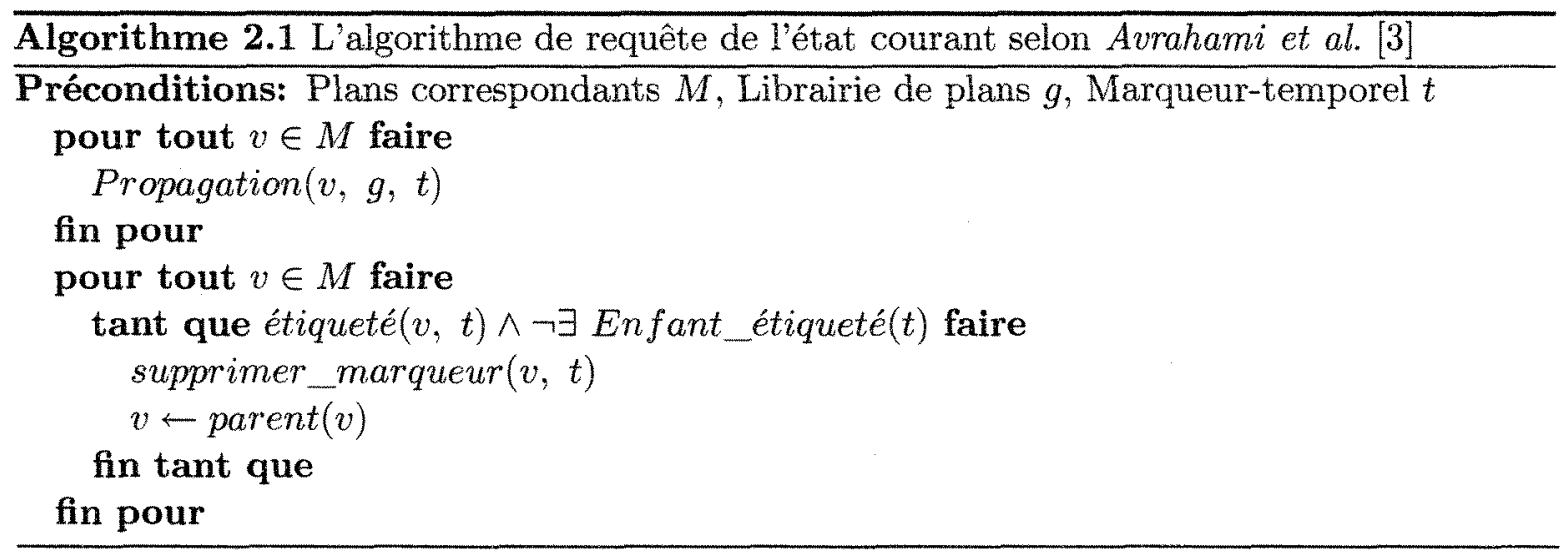

L'algorithme CSQ prend en entrées la librairie de plans et l'ensemble des plans correspondants à l'observation courante. Pour chaque plan qui correspond à l'action observée courante, l'algorithme $C S Q$ étiquette le plan avec le marqueur temporel de l'observation courante afin de garder une trace de l'ordre dans lequel les hypothèses sont formées. On utilise les arêtes séquentielles et les marqueurs temporels pour éliminer les hypothèses qui sont inconsistantes 
selon l'historique des observations. L'algorithme opère de façon suivante : à partir d'un des plans qui correspondent à l'observation courante, on parcourt le graphe vers la racine tout en étiquetant tous les nœuds qui se retrouvent sur le chemin. Si cela réussit, on propage le marqueur temporel vers le bas. Lors de la propagation du marqueur temporel dans les deux sens, le succès de la propagation est déterminé à l'aide de conditions qui vérifient la validité temporelle de l'hypothèse. Il existe deux cas :

1. le nœud en question suit une arête séquentielle à partir d'un plan qui était étiqueté au temps $t-1$

2. le nœud est un premier fils (aucune arête séquentielle menant à lui).

Un premier fils peut être sélectionné en tout temps (par exemple, si un autre plan a été interrompu). Si aucun des deux cas n'est applicable, alors le noud ne fait pas partie d'une hypothèse temporellement consistante, et son étiquette doit être supprimée, avec toutes les étiquettes qu'il a produites lors du parcourt vers la racine. On obtient donc un ensemble de chemins de plans complet qui constitue l'ensemble des hypothèses de l'état courant. Après la phase de propagation, l'algorithme $C S Q$ parcourt les étiquettes pour éliminer celles qui sont hiérarchiquement inconsistantes, c'est-à-dire les cas où un parent est étiqueté et qu'aucun de ses enfants ne le sont. L'algorithme $C S Q$ est appelé à chaque nouvelle observation.

La Figure 2.4 montre ce processus en action (les nombres encerclés dans la figure représentent les marqueurs de temps). En supposant que le FDT est utilisé au temps $t=1$ pour trouver plusieurs instances qui correspondent au plan position. L'algorithme de propagation débute avec quatre instances. Il échoue immédiatement l'étiquetage de l'instance qui suit dégager et approcher la balle, car celles-ci n'étaient pas étiquetés à $t=0$. L'instance de position sous compter est initialement étiquetée, mais la propagation échoue au parent compter parce qu'il suit attaquer, et attaquer n'est pas étiqueté. Ainsi, toutes les étiquettes $t=1$ seront supprimées de compter et de son fils position. Pour les deux instances restantes, la propagation réussit et on a les hypothèses racine $\rightarrow$ défendre $\rightarrow$ position et racine $\rightarrow$ attaquer $\rightarrow$ position. 


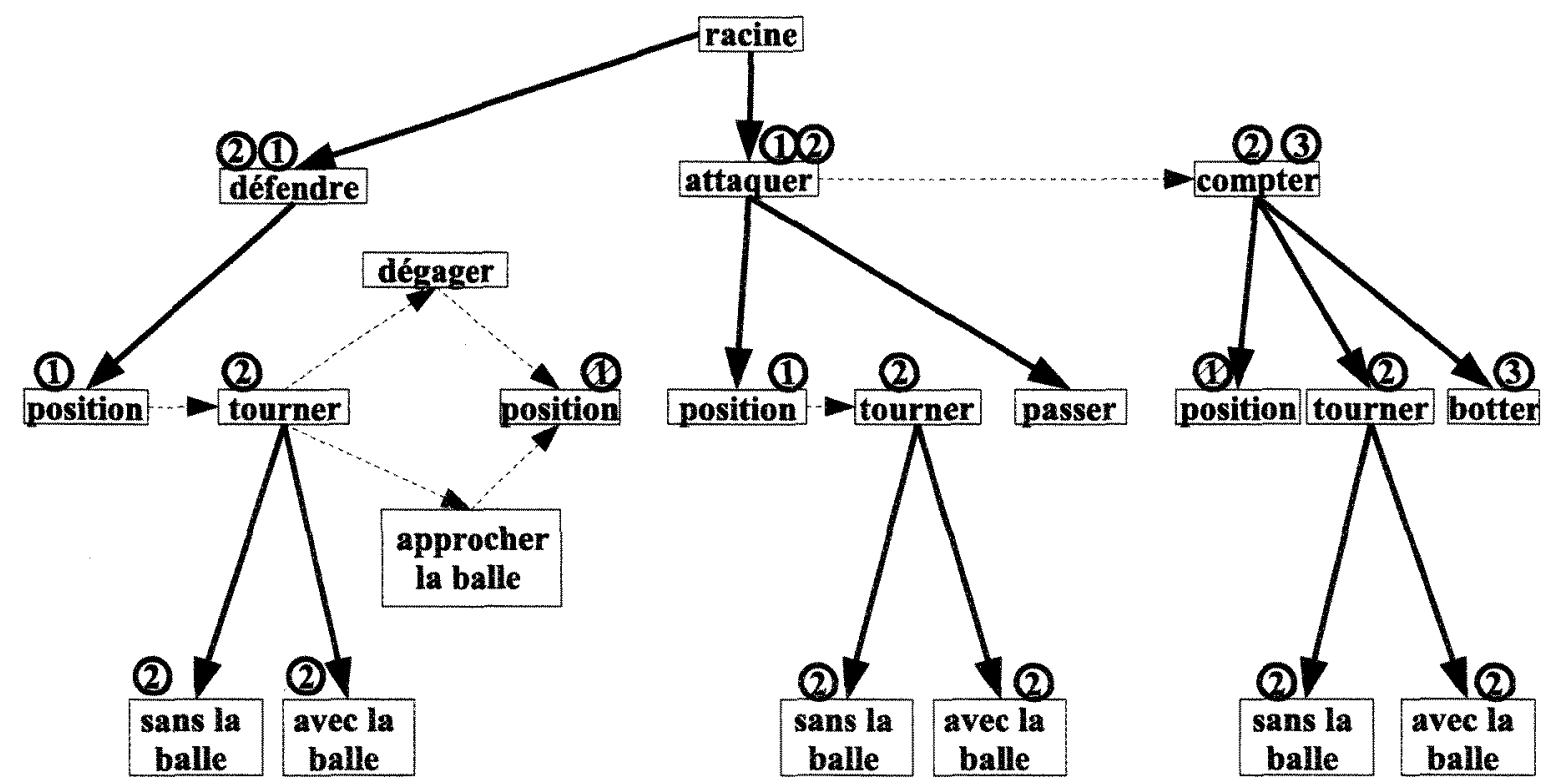

Figure 2.4 - Propagation des marqueurs temporels dans une librairie de plans selon Kaminka et al. $[52]$

Au temps $t=2$, les actions observées correspondent aux trois instances du plan tourner. L'étiquette $t=2$ se propage pour les trois instances et il y a maintenant six hypothèses (on omet le préfixe commun racine) :

- défendre $\rightarrow$ tourner $\rightarrow$ sans la balle

- défendre $\rightarrow$ tourner $\rightarrow$ avec la balle

- attaquer $\rightarrow$ tourner $\rightarrow$ sans la balle

- attaquer $\rightarrow$ tourner $\rightarrow$ avec la balle

- compter $\rightarrow$ tourner $\rightarrow$ sans la balle

- compter $\rightarrow$ tourner $\rightarrow$ avec la balle

On doit maintenant décider lequel des trois plans principaux se produit : défendre, attaquer, ou compter. Toutefois, obtenir la prochaine observation peut désambiguïser les hypothèses. Si l'on observe dégager ou approcher la balle, il sera clair que l'agent observé exécute l'hypothèse défendre. Sinon, on peut éliminer cette hypothèse. On peut alors utiliser des évidences négatives pour désambiguïser l'espace d'hypothèses, c'est-à-dire qu'une action observée au temps $t$ peut 
éliminer une hypothèse générée au temps $t-1$ si l'action observée n'est pas l'action suivante selon l'hypothèse générée.

Pour chaque plan qui correspond aux actions observées, la propagation parcoure la hauteur de la librairie de plans et peut, dans le pire cas, prendre $O\left(L^{2}\right)$ si l'on vérifie l'étiquette précédente pour chaque plan ( $L$ étant le nombre de plans dans la librairie). Dans un contexte réel, cela devrait être de $O\left(\log ^{2} L\right)$.

\subsubsection{Génération d'hypothèses}

Lorsque la librairie de plans est étiquetée par les différents marqueurs temporels, on peut générer les hypothèses pour réponde aux requêtes de reconnaissance. La génération d'hypothèses à propos de l'état courant sélectionné (le chemin de plans) est triviale : selon le dernier marqueur temporel $t_{0}$, on parcourt la librairie en identifiant les chemins de plans complets qui sont étiquetés $t=t_{0}$. L'ensemble de ces chemins de plans constitue la réponse pour ce type de requête. Toutefois, la génération d'hypothèses sur la séquence de plans qui ont été sélectionnés par l'agent à partir de $t=0$ jusqu'à maintenant ne consiste pas à simplement énumérer les combinaisons des requêtes pour les temps $t=0, t=1, \ldots, t=t_{0}$. La raison est que de nouvelles hypothèses générées au temps $t_{0}$ peuvent éliminer des hypothèses qui étaient valides aux temps $t<t_{0}$, en exploitant l'échec d'observation des plans prévus.

Supposons que l'on ait fait les observations au temps $t=1$ et $t=2$ de l'exemple précédant. Si au temps $t=3$ les observations correspondent au plan botter. Le plan compter est le seul plan consistant avec l'étiquette $t=3$, même si défendre et attaquer sont étiquetés aux temps $t=[1,2]$. Après avoir fait l'observation à l'étiquette $t=3$, on peut éliminer la possibilité que défendre ait été sélectionné par l'agent, parce que compter peut suivre seulement attaquer et que l'absence d'indices pour dégager ou approcher la balle au temps $t=3$ peut être utilisée pour l'éliminer. On infère donc que la séquence des chemins de plans qui ont été sélectionnés par l'agent est attaquer $\rightarrow$ position à $t=1$, attaquer $\rightarrow$ tourner à $t=2$ (bien que nous ne puissions pas être sûr lequel des fils de tourner a été sélectionné), et finalement compter $\rightarrow$ botter à $t=3$. 
Pour la méthode de génération des hypothèses, on utilise une structure de graphe qui maintient les hypothèses selon les marqueurs temporels. L'avantage est que pour chaque marqueur temporel $t$, on utilise cette structure pour éliminer les hypothèses étiquetées à $t-1$ qui sont devenues invalides. Cette structure consiste en un graphe connecté $G^{\prime}$ (graphe d'hypothèses), où les sommets correspondent aux chemins de plans dans la librairie de plans où l'étiquetage a réussi (les hypothèses). Les arêtes dans $G^{\prime}$ connectent les sommets d'hypothèses étiquetés $t$ aux sommets d'hypothèses étiquetés $t+1$. Le graphe $G^{\prime}$ est conséquemment construit en niveaux, où chaque niveau représente les hypothèses maintenues dans chaque marqueur temporel. Pour chaque ensemble d'hypothèses d'actions au temps $t_{0}$, on ajoute à $G^{\prime}$ un niveau $t_{0}$ contenant toutes les hypothèses possibles étiquetées $t=t_{0}$ et que sa propagation ait réussi dans la librairie de plans. Ensuite, on crée des arêtes entre les sommets $y_{1}, \ldots, y_{n}$ au niveau $t-1$ et à chaque hypothèse $x$ au niveau $t$ de la façon suivante :

1. si $x$ ne fait pas partie de la séquence (il s'agit d'un premier fils), alors on connecte chaque $y_{i}$ à $x$;

2. si $x$ fait partie d'une séquence, on connecte $y_{i}$ à $x$ si l'un des plans dans $y_{i}$ a une arête séquentielle à n'importe quels plans dans $x$;

3. si $x$ est égal à $y_{i}$, on les connecte ensemble, ce qui permet les auto-cycles.

Pour générer toutes les séquences de chemins de plan qui sont consistantes avec les actions observées, on parcourt $G^{\prime}$ de niveau en niveau, en gardant trace de tous les chemins de $G^{\prime}$ qui débutent au premier niveau pour terminer au dernier niveau (l'observation la plus récente).

Par exemple, le graphe $G^{\prime}$ de la Figure 2.5 est construit à partir de la librairie de plans de la Figure 2.2. Les chemins étiquetés au temps $t=1$ et ceux étiquetés au temps $t=2$ sont respectivement placés au premier niveau et au deuxième niveau. Pour chaque noud où $t=2$, on vérifie les nœuds qui sont appropriés à $t=1$. Par exemple, le nœud défendre $\rightarrow$ position peut être connecté aux nœuds défendre $\rightarrow$ tourner $\rightarrow$ sans la balle et défendre $\rightarrow$ tourner $\rightarrow$ avec la balle. Pareillement, attaquer $\rightarrow$ position a des arêtes vers compter $\rightarrow$ tourner $\rightarrow$ sans la balle et compter $\rightarrow$ tourner $\rightarrow$ avec la balle. On observe que, parmi les six 
hypothèses qui correspondaient aux observations jusqu'au temps $t=2$, quatre hypothèses sont éliminées une fois que l'on incorpore les observations au temps $t=3$.

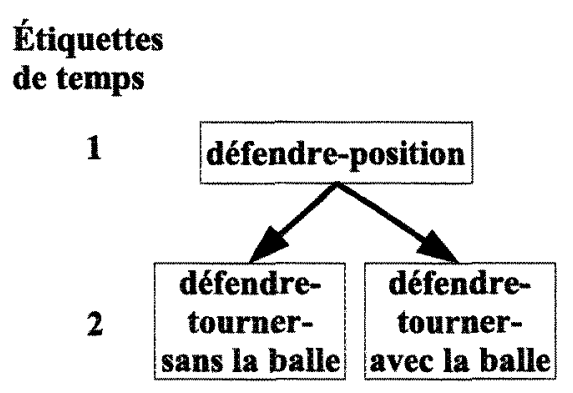

3

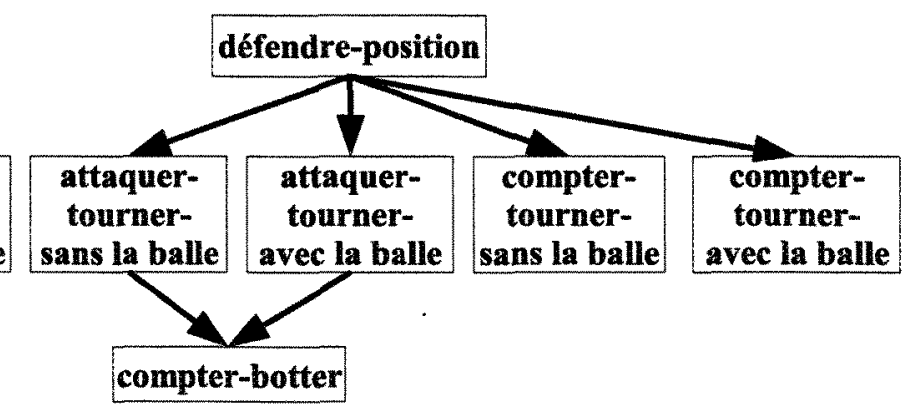

Figure 2.5 - Un exemple d'un graphe d'hypothèses $G^{\prime}$ selon Kaminka et al. [52]

Soit $L$, le pire cas pour le nombre de plans qui correspondent à une seule action observée. Pour chaque nœud dans $G^{\prime}$ avec le marqueur temporel $t$ (qui peut être au plus de $O(L)$ ), on vérifie tous les nœuds avec un marqueur temporel $t-1$ (aussi $O(L)$ ), donc on a un facteur de $L^{2}$ pour chaque niveau additionnel. Conséquemment, après $N$ observations, le temps d'exécution est, au pire, $O\left(N L^{2}\right)$.

\subsubsection{Prise en compte des plans temporels complexes}

Le modèle précédemment décrit peut être utilisé pour reconnaître les plans qui sont ordonnés dans le temps. Il permet aux plans d'avoir des cycles pour qu'ils ne soient pas instantanés. Toutefois, ce modèle n'est pas en mesure de reconnaître les formes les plus complexes de plans temporels, comme maintenir la sélection d'un plan à l'intérieur d'un intervalle borné, ou en interrompant une séquence de plans sous un nœud, pour en exécuter un autre, et d'y retourner plus tard (entrecroisement de plans). Les deux prochains points montrent comment étendre l'algorithme CSQ afin de tenir compte de ces plans temporels.

\subsubsection{Gestion de la durée}

Les instances d'un même plan peuvent varier dans la durée de leur exécution. Par exemple, en fonction de la distance de la balle, un agent observé peut exécuter lentement ou 
rapidement le plan approcher la balle. Comme résultat, on peut avoir plusieurs marqueurs temporels d'observation $(t, t+1, \ldots, t+k)$ qui sont consistants avec un seul plan et reflète par conséquent la durée que son exécution exige entre 1 et $k+1$ marqueurs temporels. Toutefois, il arrive souvent que des intervalles sur la durée d'exécution soit connus. On doit alors tenir compte des contraintes sur la durée d'exécution des plans. Pour ce faire, le mécanisme d'étiquetage est étendu pour gérer deux types d'étiquettes:

1. Les étiquettes rigides signifient qu'un plan est (ou n'est pas) consistant en respect aux précédents plans, et est aussi consistant avec les contraintes de durée (temps minimum et maximum).

2. Les étiquettes molles signifient qu'un plan est (ou n'est pas) consistant avec les observations (et avec les plans antérieurs), mais n'est pas à l'intérieur de ses contraintes de durée.

Donc, lors de la vérification de la consistance d'un plan, on vérifie si ce plan est consistant avec les observations et les plans précédents ainsi qu'avec les contraintes temporelles. Alors, les chemins de plans complets considérés comme hypothèses sont uniquement constitués d'étiquettes rigides. Pour calculer la durée d'un plan, on prend les marqueurs temporels du plan et on compte le nombre de marqueurs consécutifs à partir du marqueur $t$. Par exemple, si un plan est étiqueté avec les marqueurs $t=1, t=4, t=5$, et $t=6$, alors la durée est de 3.

\subsubsection{Les plans entrecroisés}

Un agent peut poursuivre plusieurs buts en entrecroisant les étapes (actions) de plusieurs plans. L'agent observé peut commencer un plan, interrompre son exécution pour débuter celle d'un autre plan, puis par la suite effectuer les étapes restantes du premier plan. Pour pouvoir tenir compte de l'entrecroisement de plans, on ajoute un drapeau de mémoire à chaque premier nœud fils d'un plan. Ce drapeau contient la dernière étiquette de marqueur temporel dans la chaîne séquentielle à partir de ce fils. On utilise le drapeau pour éliminer des plans qui sont au milieu de la chaîne et qui ne sont pas ceux où l'on a fait une pause. Cela permet 
de retourner plus tard au plan qui suit celui où l'on a fait une pause. Donc, un plan reste consistant s'il continue une séquence de plans qui avait été interrompue. Pour augmenter l'efficacité de l'algorithme, on peut spécifier quels sont les plans qui peuvent être interrompus et la limite de temps qu'un plan peut être interrompu pour qu'il soit encore valide.

\subsubsection{Manipulation des observations avec perte}

Un autre problème avec le modèle précédemment présenté est la perte de propriétés d'observation causée par des défaillances intermittentes. Cela peut causer une absence de propriétés dans une observation ou une absence de l'observation (toutes les propriétés ne sont pas perçues). Les deux prochaines sections présentent le traitement de ces types d'absences d'information.

\subsubsection{Perte de propriétés d'une observation}

Chaque observation est constituée d'un tuple de propriétés observables (état de l'environnement, actions prises, conditions d'exécution maintenues). On suppose généralement que toutes les propriétés pertinentes sont toujours observables, ce qui n'est pas réaliste dans un contexte réel, où certaines propriétés sont par intermittence non observables (défaillances techniques, erreurs de communication, ...). Donc, s'il y a une ou des propriétés manquantes, la phase de correspondance échouera parce que le FDT ne tient pas compte de l'absence de propriétés. Pour pallier ce problème, on utilise l'arbre de décision des propriétés manquantes ou LFDT ("Lossy Feature Decision Tree »), une version améliorée du FDT qui tient compte des propriétés manquantes. La représentation du LFDT est similaire au FDT, à l'exception que pour chaque nœud, on ajoute une branche supplémentaire qui représente une valeur manquante (absent tel qu'illustré dans la Figure 2.6). Lors de la construction du LFDT, tous les plans qui sont consistants à un noud (lesquels sont divisés selon la valeur de la propriété associée au nœud) sont passés à la branche de l'absence de valeur. Par conséquent, lorsque l'on parcourt le $L F D T$ durant la phase de correspondance, si l'on rencontre une propriété où la valeur est manquante, on suit la branche absent. À l'exception de la présence de branche absent, le processus se déroule de la même façon qu'avec le FDT. 


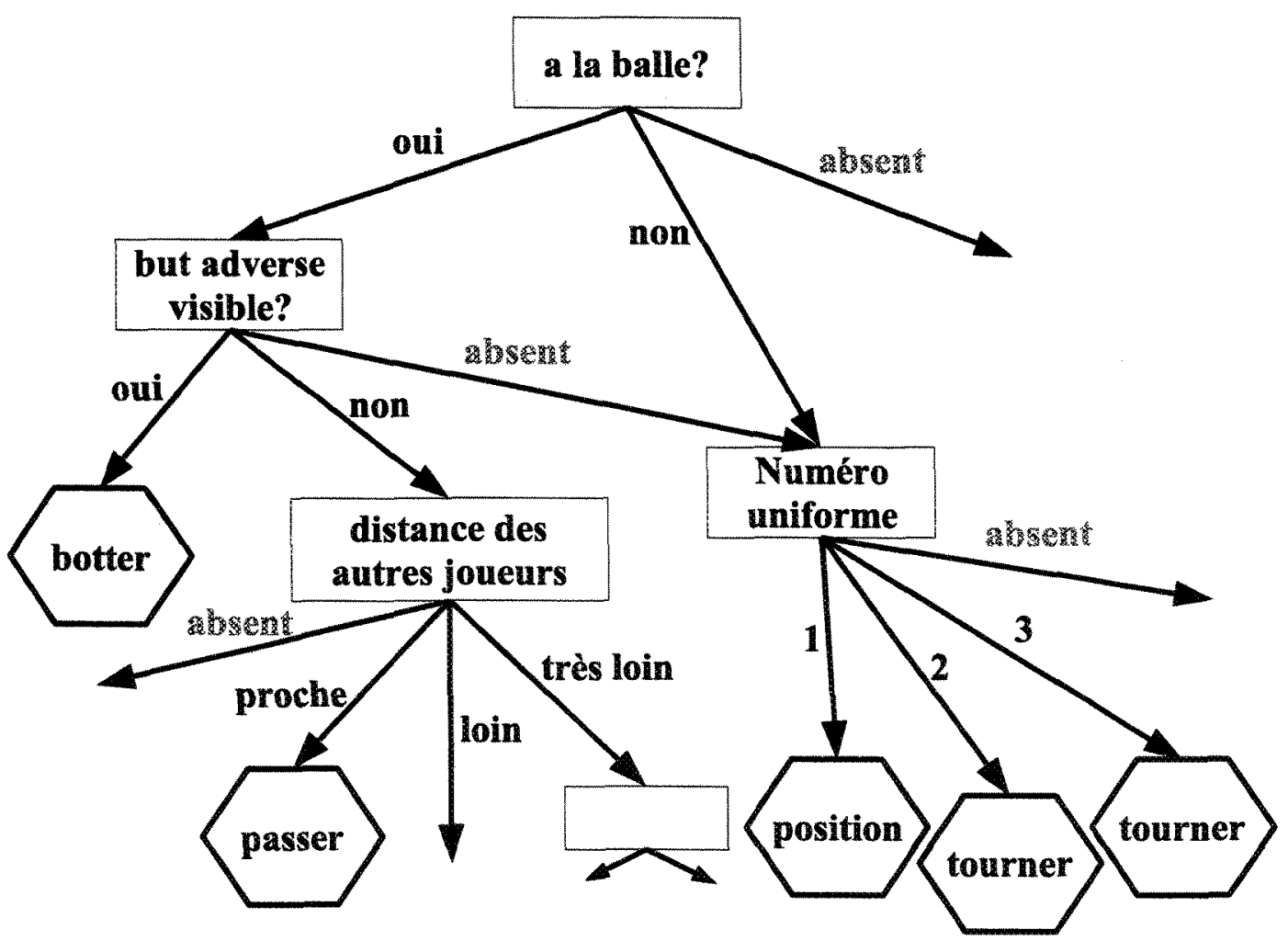

Figure 2.6 - Un exemple d'un arbre de décision des propriétés manquantes selon Avrahami et al. [5]

Un autre moyen de régler ce type de problème est d'utiliser le FDT de tel sorte que lorsque l'on rencontre une propriété où la valeur est manquante, on parcourt toutes les branches de cette propriété et on collecte les pointeurs résultants de chacun des chemins. Cette méthode permet d'améliorer la rapidité de l'algorithme de correspondance comparativement à la méthode par LFDT, mais on se retrouve avec un problème d'espace.

\subsubsection{Perte d'une observation}

On suppose généralement que tout changement de l'état interne (changement dans le chemin de plans) est reflété dans les observations. Dans un contexte réaliste, certaines prises de décisions internes ne sont pas (ou partiellement) observables, pour tous les plans le long d'un chemin de décomposition de plan spécifique. Donc, dans ce cas, l'observation est manquante (toutes les propriétés ne sont pas observables). Pour répondre à ce problème, on 
peut modifier la phase de propagation. On marque les plans potentiellement non observables avec une étiquette absent dans la libraire. Toutefois, les premiers et derniers plans dans une séquence de plans peuvent ne pas avoir cette étiquette. Lors de la propagation, tous les nœuds étiquetés absent et qui sont dans une séquence seront sautés (s'ils ne sont pas étiquetés par un marqueur temporel) quand la séquence est vérifiée pour la consistance temporelle. L'utilisation de cette méthode doit être soigneuse parce qu'elle peut influencer de manière significative le temps d'exécution et le nombre d'hypothèses.

\subsubsection{Extension probabiliste}

Pour mieux désambiguïser l'ensemble des hypothèses sur l'état courant de l'agent observé obtenues à l'aide du modèle que nous venons de présenter, on sélectionne des hypothèses en fonction de leurs probabilités a posteriori découlant de l'observation des actions. Chacune de ces hypothèses est alors une hypothèse probable sur l'état courant. L'extension probabiliste [4] est basée sur le modèle de Markov caché hiérarchique [36] ou HHMM («Hierarchical Hidden Markov Model ») pour représenter l'information probabiliste de la librairie de plans. Les HHMM sont des processus stochastiques structurés en plusieurs niveaux, et constituent une généralisation des modèles de Markov cachés [75,76], où chacun des états cachés est un modèle probabiliste autonome (chaque état est aussi un $H H M M$ ).

Chaque plan dans la librairie de plans est dénoté par $q_{i}^{d}$, où $i$ est l'index du plan et $d$ est la profondeur dans la hiérarchie (l'index de profondeur pour la racine est 1 et l'index du dernier niveau est $D$ ). Pour chaque plan $q_{i}^{d}$ dans la librairie de plans, on définit trois probabilités différentes.

La première probabilité est la probabilité de suivre une arête séquentielle du $i^{e}$ plan au $j^{e}$ plan. Cela exprime comment il est probable que l'agent complète l'exécution du plan courant et va au prochain plan dans la séquence. Pour chaque état interne $q_{i}^{d}$, il y a une matrice

de probabilités des transitions d'états dénotée $A^{q^{d}}=\left(a_{i, j}^{q^{d}}\right)$, où $\left(a_{i, j}^{q^{d}}\right)=P\left(q_{j}^{d+1} \mid q_{i}^{d+1}\right)$ est la probabilité de faire une transition horizontale du $i^{\epsilon}$ plan au $j^{e}$ plan. Les arêtes de cycles peuvent aussi être représentées par une matrice des arêtes séquentielles probabilistes. 
La deuxième probabilité caractérise la possibilité que l'agent interrompe l'exécution du plan courant et donne le contrôle au plan parent ou de niveau supérieur. On représente cette probabilité par $a_{i, f i n}^{q^{d}}$, qui est la probabilité que le plan $i$ aille à l'état $f i n$ et retourne le contrôle à son parent $q_{i}^{d}$.

La troisième probabilité est la transition verticale, caractérisant la possibilité que l'agent exécute chacun des premiers nœuds fils du plan (les nœuds fils directs). Cette probabilité est représentée par $\Pi^{q^{d}}=\pi^{q^{d}}\left(q^{d+1}\right)=P\left(q_{i}^{d+1} \mid q^{d+1}\right)$, qui est la probabilité que le plan $q^{d}$ ait initialement activé le plan $q_{i}^{d+1}$. Il est aussi possible de représenter la durée probable d'un plan.

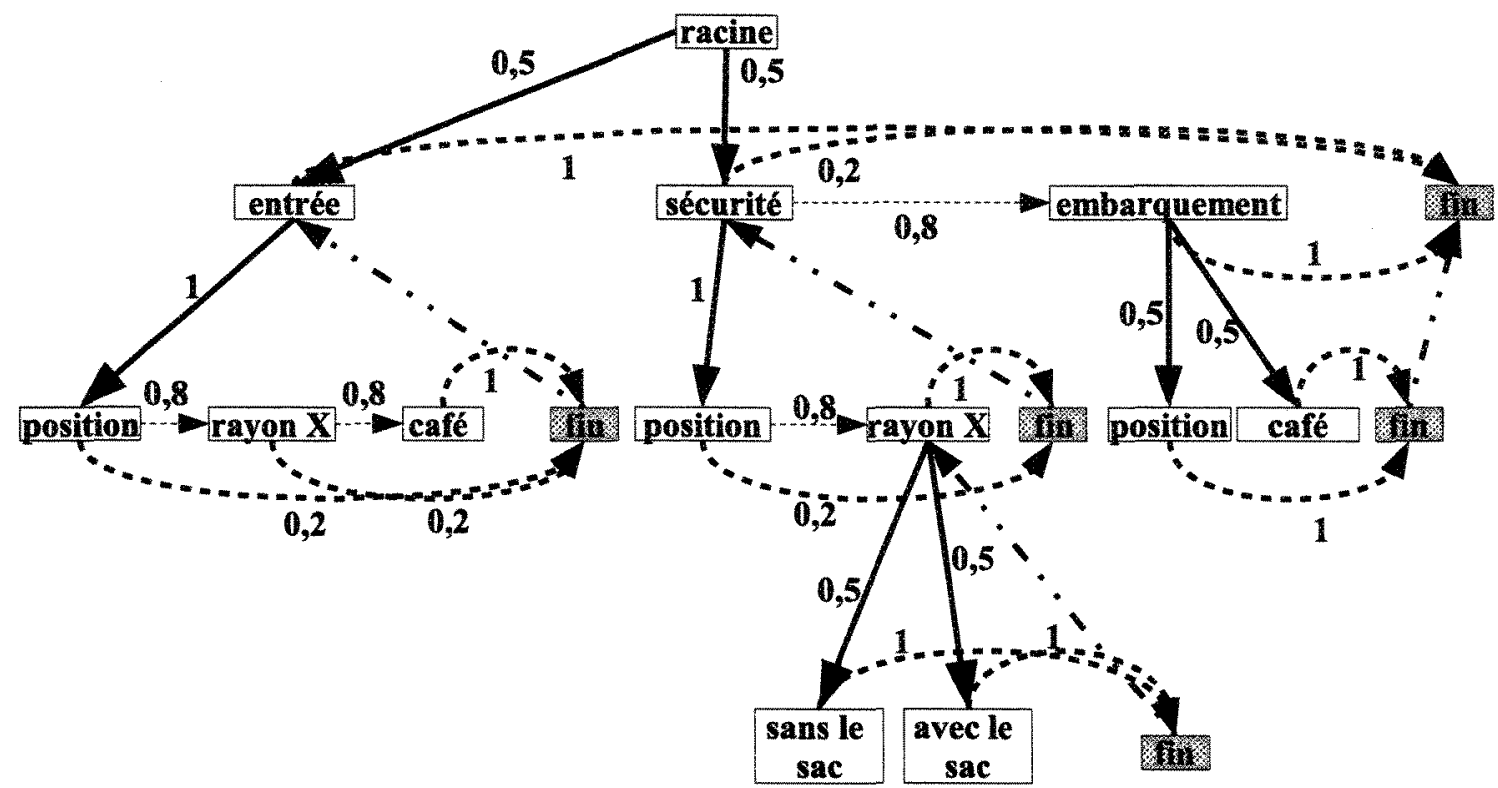

Figure 2.7 - Un exemple d'une librairie de plans représentée en $H H M M$ modifié de Avrahami et al. [4]

La Figure 2.7 montre une portion de la librairie de plans après sa conversion en $H H M M$. En premier lieu, on ajoute un état fin pour chaque niveau et une arête de chaque plan vers cet état. Cette arête représente la probabilité d'interruption. Chaque plan doit aussi avoir une arête de cycles (absente pour clarifier l'exemple). On utilise ces probabilités pour évaluer les hypothèses qui ont été obtenues par l'algorithme symbolique. Pour calculer la probabilité pour chaque hypothèse, on parcourt la librairie de plans à partir de la feuille de chacune des 
hypothèses sélectionnées au temps $t-1$, jusqu'à la feuille de chacun des hypothèses de l'état courant au temps $t$. Lorsqu'on parcourt la librairie de plans, on multiplie les probabilités appropriées (continuation ou interruption ou déplacement au prochain) par chacun des plans à partir de la feuille du chemin précédent à la feuille du chemin courant. S'il y a plusieurs façons pour passer de la feuille de l'hypothèse précédente vers la feuille de l'hypothèse courante, il faut alors évaluer chacune d'entre-elles et sélectionner la meilleure.

Formellement, l'algorithme symbolique a filtré toutes les hypothèses précédentes (chemins) $W=W_{1}, W_{2}, \ldots, W_{r}$, et les hypothèses possibles courantes (chemins) $X=X_{1}, X_{2}, \ldots$, $X_{l}$, basées sur les hypothèses d'actions. Pour déterminer la meilleure hypothèse, on doit évaluer pour chaque plan plausible la probabilité $P\left(X_{i}, t+1\right.$ observations $)$, qui représente la probabilité d'avoir le chemin $X_{i}$ au marqueur temporel $t+1$ sachant la séquence d'observations. En assumant la propriété de Markov, qui consiste à dire que les états futurs sont indépendants aux états passés sachant l'état présent [82], on obtient la formule suivante :

$$
\begin{aligned}
\hat{X}_{i} & =\arg \max _{X_{i}} P\left(X_{i} \mid \text { observations }\right) \\
& =\arg \max _{X_{i}} \sum_{W_{k} \in W} P\left(W_{k}\right) \times P\left(X_{i} \mid W_{k}\right)
\end{aligned}
$$

Pour estimer cette probabilité, on utilise la valeur probabiliste calculée dans le marqueur temporel précédent $P\left(W_{k}\right)$ et on la multiplie par $P\left(X_{i} \mid W_{k}\right)$ pour chaque chemin courant possible $X_{i}$, où $X_{i}=x_{i}^{1}, \ldots, x_{i}^{m}$ et le chemin précédent $W_{k}=w_{k}^{1}, \ldots, x_{k}^{n}$ (l'index supérieur représente la profondeur dans la librairie de plans). L'index du plan a été omis pour clarifier la présentation.

Le calcul de cette probabilité est effectué dans deux cas, pour $w \in W$ et $x \in X$ donnés. Dans le premier cas, $x$ et $w$ ont un parent commun, et $x$ est un chemin formé par les arêtes des premiers fils de ce parent commun. On infère alors la probabilité en parcourant les nœuds dans $w$ vers le haut (en prenant les arêtes interruption) jusqu'à ce que nous atteignons un parent commun à $x$ et $w$, et ensuite on parcourt la librairie vers le bas (en prenant les arêtes 
de décomposition des premiers fils) jusqu'à $x$. Dans le deuxième cas, $x$ est atteint en suivant une arête séquentielle à partir d'un nœud dans $w$ vers un nœud dans $x$.

Dans les deux cas, la probabilité du parcourt vers le haut à partir d'une feuille $g^{n}$ d'une profondeur $n$ vers un parent $g^{j}$ (où $j<n$ ) est donnée par:

$$
\gamma_{g}^{j}=\prod_{d=n}^{j} a_{g, f i n}^{d}
$$

et la probabilité du parcourt vers le bas à partir d'un parent $g^{j}$ à une feuille $g^{m}$ est comme suit :

$$
\beta_{g}^{j}=\prod_{i=j}^{m} \pi^{g^{i}}\left(g^{i+1}\right)
$$

On note que l'index du plan a été omis et que seul l'index de profondeur est montré.

En utilisant $\gamma_{w}^{j}$ et $\beta_{x}^{j}$ et additionnant tous les $j$ possibles, on peut calculer la probabilité pour les deux cas où un déplacement de $w_{n}$ vers $x_{m}$ est possible. Ceci est donné par:

$$
\begin{aligned}
P\left(X_{i} \mid W_{k}\right) & =\sum_{j=n-1}^{1}\left[\gamma_{w}^{j} \times \operatorname{Eq}\left(x^{j}, w^{j}\right) \times \beta_{x}^{j}+\gamma_{w}^{j} \times a_{w, x}^{j} \times \beta_{x}^{j}\right] \\
& =\sum_{j=n-1}^{1} \gamma_{w}^{j} \beta_{x}^{j}\left[\mathrm{Eq}\left(x^{j}, w^{j}\right)+a_{w, x}^{j}\right]
\end{aligned}
$$

Le premier cas est couvert avec le terme $\gamma_{w}^{j} \times \mathrm{Eq}\left(x^{j}, w^{j}\right) \times \beta_{x}^{j}$, où la fonction $\mathrm{Eq}\left(x^{j}, w^{j}\right)$ retourne 1 si $x^{j}=w^{j}$, et 0 sinon. Le calcul de la probabilité parcourt toutes les manières d'interrompre un plan, grimpe vers un parent commun, et suit les décompositions du premier fils vers un nouveau plan. Le second cas est couvert par le terme $\gamma_{w}^{j} \times a_{w, x}^{j} \times \beta_{x}^{j}$, où $a_{w, x}^{j}$ retourne la probabilité de prendre une arête séquentielle de $w^{j}$ à $x^{j}$ (si une telle arête existe) et que l'agent ait fini de $w^{j}$.

\subsubsection{Exemple}

Pour montrer le processus de la Figure 2.7, supposons que l'algorithme symbolique fait correspondre au temps $t=6$ les instances du plan café et au temps $t=5$ les 
instances du plan rayon $X$. Pour calculer la probabilité de $P($ embarquement $\rightarrow$ café|sécurité $\rightarrow$ rayon $X$ ), on parcourt l'arbre et on multiplie les probabilités des arêtes rencontrées. On obtient $P($ embarquement $\rightarrow$ café $\mid$ sécurité $\rightarrow$ rayon $X)=1 \times 0.8 \times 0.5=0.4$ (en interrompant rayon $X$ de sécurité, en suivant l'arête séquentielle vers embarquement, et en choisissant café). On obtient $P($ entrée $\rightarrow$ café|entrée $\rightarrow$ rayon $X)=0.8$ (en suivant l'arête séquentielle de rayon $X$ vers café). Par conséquent, l'option la plus probable est que l'agent boit un café dans l'aire d'entrée et non dans l'aire d'embarquement. On doit noter que ces résultats doivent être multipliés par $P($ sécurité $\rightarrow$ rayon $X)$ et $P($ entrée $\rightarrow$ rayon $X)$. Ces probabilités ont été considérées égales et elles ont donc été omises.

\subsubsection{Bilan des travaux de Avrahami et al.}

Lors du processus de reconnaissance, les travaux de Avrahami et al. tiennent compte de l'historique et de la durée des actions grâce à des marqueurs temporels dans la librairie de plans. Cela permet, lors de la génération des hypothèses, d'écarter les hypothèses qui sont temporellement inconsistantes. L'utilisation d'un arbre de décision permet de faire correspondre les observations avec une étape d'un plan particulier dans la librairie, même si les caractéristiques utilisées dans l'arbre de décision sont partiellement observables. L'utilisation de marqueurs d'interruption dans la librairie de plans permet de tenir compte des plans entrecroisés, mais cette manière de procéder n'est pas efficace car il faut vérifier s'il existe un lien de séquence avec chaque nouvelle action et le ou les plans interrompus. Contrairement à ce qu'il est supposé ici, une interruption d'un plan peut être la conséquence d'une exécution erronée d'un plan. Dans un contexte où l'on suppose la rationalité de l'agent observé, la meilleure explication d'une interruption est que l'agent exécute des plans entrecroisés. Dans un contexte réel, cette supposition de rationalité est irréaliste, car il est possible qu'un agent observé (humain) fasse des erreurs. La conversion de la librairie de plans en un HHMM permet de désambiguiser les différentes hypothèses générées. Cependant, il est difficile de bien évaluer les probabilités des différentes transitions dans la librairie de plans pour s'assurer de la bonne quantification probabiliste des hypothèses. Aussi, on suppose que les hypothèses générées sont 
parmi les plans de la librairie de plans et donc, on ne peut pas tenir compte des plans erronés, qui sont absents de la libraire.

\subsubsection{Les travaux de Geib et al.}

Les travaux de Geib et al. $[37,38,39,40,44]$ proposent un modèle de reconnaissance de plans basé sur un raisonnement abductif probabiliste. Un raisonnement de type abductif [46] est un processus d'inférence non monotonique qui permet de construire des hypothèses permettant d'interpréter un ensemble d'événements observés [32].

\subsubsection{Représentation de la librairie de plans}

Le modèle de reconnaissance proposé dans les travaux de Geib et al. utilise une librairie de plans basée sur les réseaux hiérarchiques de tâches ( Hierarchical Task Network » ou $H T N$ ) [34]. Par exemple, la Figure 2.8 représente une portion de la librairie de plans d'une hypothétique station spatiale.

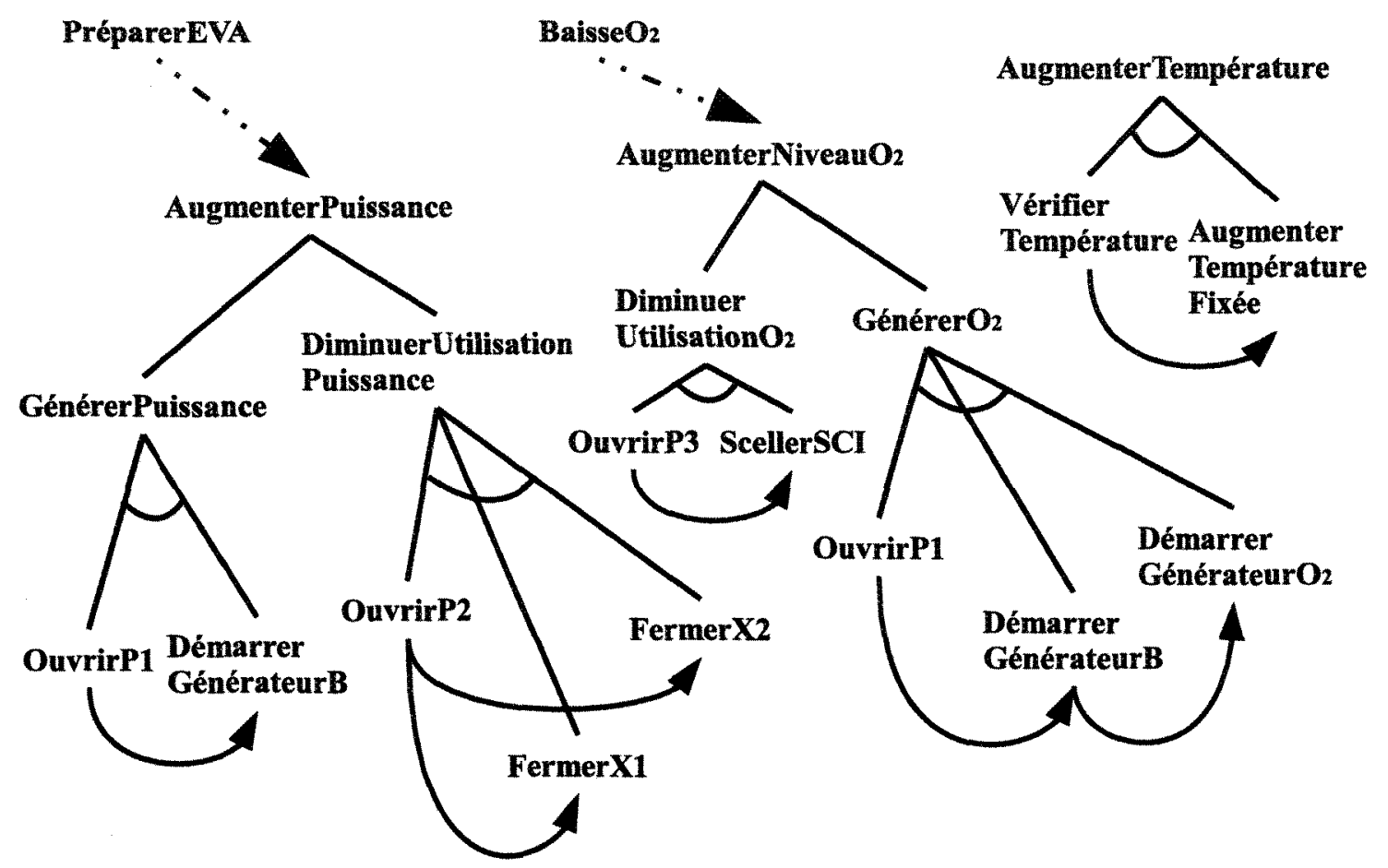

Figure 2.8 - Une librairie de plans hiérarchique selon Goldman et al. [44] 
Quand l'agent observé veut atteindre un but, comme l'augmentation de la puissance (AugmenterPuissance), la librairie de plans fournit un ensemble de méthodes alternatives que l'agent peut utiliser. L'augmentation de la puissance peut s'accomplir soit en générant plus de puissance (générer - puissance) ou soit en diminuant la consommation de la puissance (DiminuerUtilisationPuissance). Pour pouvoir diminuer la consommation de la puissance, l'agent observé doit effectuer trois étapes : ouvrir le panneau d'accès 2 (OuvrirP2), fermer le sous-système d'expérimentation $X 1$ (Fermer X1), et fermer le sous-système d'expérimentation $X 2$ (Fermer $X 2)$. On peut considérer la librairie de plan comme un arbre $E T / O U$, avec les buts comme des nœuds de type $O U$ et les méthodes comme les nœuds de type ET. Dans la librairie de plans de la Figure 2.8, les buts sont : augmenter la puissance, augmenter le niveau d'oxygène, et augmenter la température. Les méthodes alternatives pour augmenter la puissance sont générer de la puissance et diminuer l'utilisation de la puissance, et les méthodes alternatives pour augmenter le niveau d'oxygène sont diminuer l'utilisation de l'oxygène et générer de l'oxygène. Parce qu'il y a une seule façon d'augmenter la température, on n'a pas besoin d'un nœud de méthode.

Chacun des nœuds de méthode-plan possède un ensemble de fils connectés par des arcs ET représentant les actions dans les étapes de la méthode. Dans la libraire de la Figure 2.8, toutes les méthodes sont composées d'actions primitives qui sont : ouvrir le panneau d'accès 1 , allumer le générateur $B$, ouvrir le panneau d'accès 2 , fermer le sous-système d'expérimentation $X 1$, fermer le sous-système d'expérimentation X2, ouvrir le panneau d'accès 3 , allumer le générateur d'oxygène, sceller le module scientifique, vérifier la température, et augmenter le thermostat. En général, les méthodes peuvent introduire de nouveaux buts. Dans plusieurs cas, les étapes d'une méthode doivent être accomplies dans un ordre particulier, mais l'agent possède tout de même un peu de liberté dans le choix de l'ordre de ses actions. Les contraintes d'ordonnancement sont représentées par des flèches sur la Figure 2.8.

Les actions exécutées peuvent être présentes dans plusieurs méthodes différentes. Par exemple, générer de la puissance et générer de l'oxygène utilisent tous les deux ouvrir le panneau d'accès 1 et allumer le générateur $B$. On peut noter que des conditions ou évènements 
peuvent être liés à certains buts. Ces conditions (représentées par les flèches pointillées dans la Figure 2.8) affectent la plausibilité de leurs buts respectifs. Par exemple, si l'agent observe une diminution dans le niveau de l'oxygène, il est plausible qu'il choisisse d'augmenter le niveau d'oxygène comme but.

\subsubsection{Le modèle d'exécution de plans}

Le modèle présenté dans [44] répond aux difficultés suivantes:

1. les plans partiellement ordonnés et les plans entrecroisés;

2. les indices à partir de l'échec à observer des actions;

3. l'influence contextuelle sur le choix des plans;

4. les domaines où le système de reconnaissance de plans et l'agent observé agissent tous les deux.

Dans le modèle d'exécution de plans, l'agent observé, au début de l'épisode de reconnaissance, choisit un ensemble de plans à exécuter, formant ainsi ses intentions. Cet ensemble détermine le groupe d'actions primitives qui sont en suspens. Tout le long de l'épisode de reconnaissance, l'agent exécute à plusieurs reprises une action en suspens, et génère un nouvel ensemble d'actions en suspens à travers lequel d'autres actions seront choisies. Ce nouvel ensemble d'action en suspens est généré à partir du groupe précédent en enlevant l'action exécutée et en ajoutant des nouvelles actions disponibles à partir des plans initialement sélectionnés. Les actions deviennent disponibles lorsque leurs prédécesseurs sont complétés. Ce processus est montré à la Figure 2.9.

Le modèle d'exécution de plans fournit un modèle conceptuel pour la génération des traces d'exécution. Pour la reconnaissance de plans, on utilise ce modèle conjointement avec le raisonnement abductif.

\subsubsection{Formalisation du modèle}

Le modèle d'exécution de plans est basé sur l'abduction probabiliste de Horn [73] ou PHA ( Probabilistic Horn Abduction »). Le PHA fournit des règles logiques impliquant des 


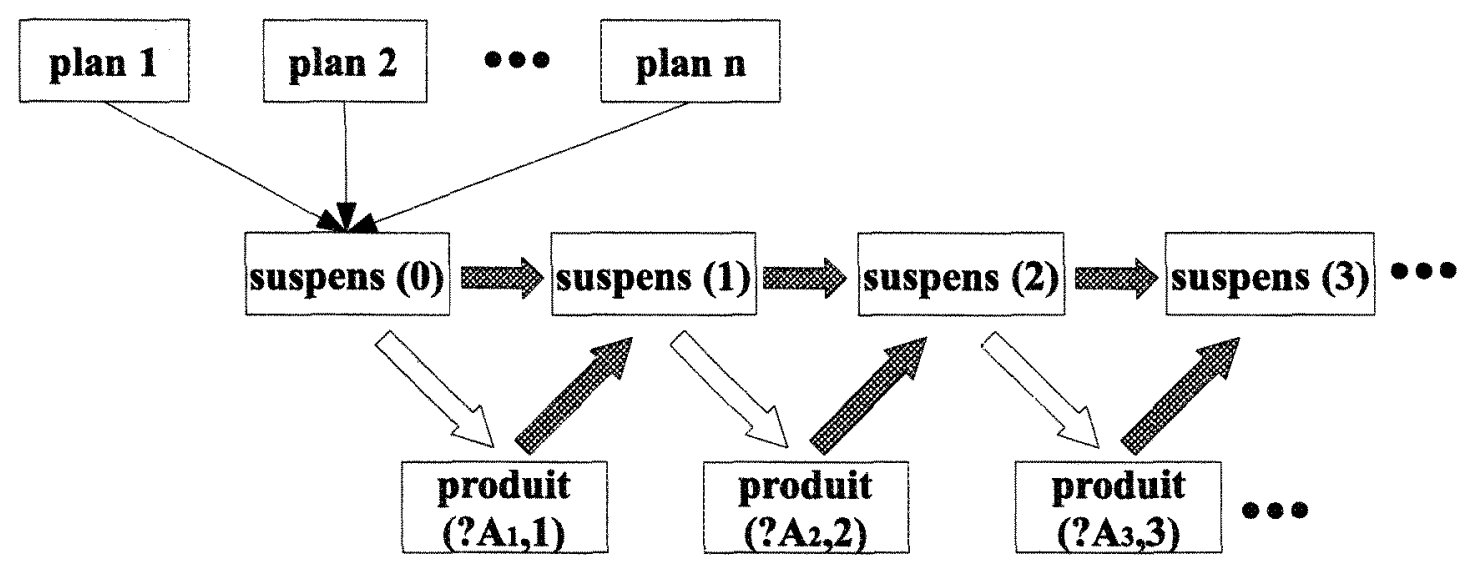

Figure 2.9 - Un réseau de croyances dynamique illustrant le modèle d'exécution de plans selon Goldman et al. [44]

propositions et des propositions distinguées supposables (les hypothèses). Ces règles sont sous la forme de clause de Horn à la Prolog $[41,82]$. Une explication pour une proposition est un ensemble d'hypothèses qui, prises avec les règles, entraînent cette proposition. Chaque hypothèse possède une probabilité a priori, de sorte que cette logique supporte la notion de la meilleure (la plus plausible) explication.

Le modèle d'exécution de plans fonctionne avec la librairie des plans que l'agent a la possibilité d'exécuter. Cette librairie est composée de tâches qui peuvent être des buts (les nœuds disjonctifs dans le graphe de la librairie) ou des méthodes (les nœuds conjonctifs dans le graphe de la librairie).

Par chaînage arrière à partir de l'observation, on doit expliquer comment l'action observée est choisie à partir des actions en suspens. On doit ensuite expliquer comment l'ensemble des actions est assemblé. L'explication de l'ensemble en suspens couvre le choix des sous-tâches comme moyens d'atteindre des buts de plus haut niveau et couvre l'évolution d'un épisode d'exécution de plans dans le temps.

Afin de prendre en compte le fait que l'agent observé peut effectuer des plans multiples, entrecroisés et partiellement ordonnés, il est stipulé qu'une action qui se produit à tout moment est nécessairement choisie à partir de l'ensemble des actions en suspens. Dans la Figure 2.9, les 
flèches vides représentent l'opération de sélection à partir de l'ensemble en suspens courant.

$$
\begin{aligned}
\text { produit }(X, T+1) \leftarrow & \text { suspens }(P, T), \\
& X \in P, \\
& \text { †sélectionné }(X, P, T+1) .
\end{aligned}
$$

La règle 2.5 indique que l'action $X$ se produit au temps $T+1$ si $X$ est dans l'ensemble des actions en suspens $P$ et est sélectionnée à partir de cet ensemble. Le littéral $\dagger$ sélectionné $(X, P, T+1)$ est l'une des propositions d'hypothèses distinguées avec une probabilité associée. Dans la présentation des règles, les hypothèses sont représentées par le symbole $\uparrow$. Actuellement, il est supposé que toutes les actions en suspens sont équiprobables. On peut utiliser un modèle plus complexe pour le choix de la prochaine action à exécuter comme, par exemple, une probabilité qui augmente en fonction du temps que l'action reste en suspens.

L'ensemble en suspens est défini récursivement, d'abord en fournissant un cas de base qui permet d'expliquer le contenu de cet ensemble au temps zéro. Dans la Figure 2.9, les flèches étroites montrent le processus de sélection de l'ensemble en suspens initial à partir de la librairie de plans. L'ensemble en suspens est sélectionné à partir de l'ensemble des actions primitives (les feuilles) :

$$
\begin{aligned}
\text { suspens }(L, 0) \leftarrow & \text { feuilles }(L s) \\
& \text { sélection_feuilles }(L s, L) .
\end{aligned}
$$

La clause pour sélection_feuilles choisit les feuilles qui sont activées (activée).

\subsubsection{Activation d'une action}

Le cœur du modèle d'exécution de plans porte sur l'activation des actions. Il y a deux raisons pour lesquelles une action peut être activée : soit qu'elle est auto-motivée (2.7) (n'est pas exécutée comme une sous-partie d'un plan), soit qu'elle est exécutée comme une partie d'un plan parent (2.8).

$$
\operatorname{activée}(A, T) \leftarrow \text { pour_elle }(A, T)
$$




$$
\begin{aligned}
\operatorname{activée}(A, T) \leftarrow & \neg \text { pour_elle }(A, T), \\
& \text { pour_parent }(A, T) .
\end{aligned}
$$

Le cas le plus simple d'activation est qu'une action est exécutée pour elle-même (pas au service d'autres buts) :

$$
\begin{aligned}
\text { pour_elle }(A, T)- & \text { pouvoir_intention }(A), \\
& \text { †avoir_intention }(A, T) .
\end{aligned}
$$

L'action $A$ doit faire partie de l'ensemble des actions qui peuvent être exécutées pour ellesmêmes (pouvoir_intention). Il n'existe pas de contrainte sur le type d'actions pouvant être exécutées pour elles-mêmes (buts, méthodes et actions primitives). En plus de pouvoir s'exécuter pour elle-même, l'action doit s'exécuter pour elle-même (avoir_intention). On note, également, qu'on doit évaluer la possibilité que l'action $A$ doit s'exécuter pour elle-même.

L'autre cas de figure consiste en l'activation d'une action en tant qu'élément d'un plan de plus haut niveau :

$$
\begin{aligned}
\operatorname{pour} \operatorname{parent}(A, T) \leftarrow & \operatorname{parents}(A, P s) \\
& \exists P \in P s \mid \text { activée_par }(A, P, T) .
\end{aligned}
$$

Il y a deux façons pour qu'une action enfant $C$ puisse être activée en tant qu'élément d'un plan parent (règles 2.11 et 2.14). Premièrement, le plan parent peut être une méthode (un ensemble d'étapes). Dans ce cas, pour activer une action particulière, celle-ci doit être un membre de l'ensemble d'étapes de la méthode et toutes les étapes préalables dans cette méthode doivent être déjà exécutées :

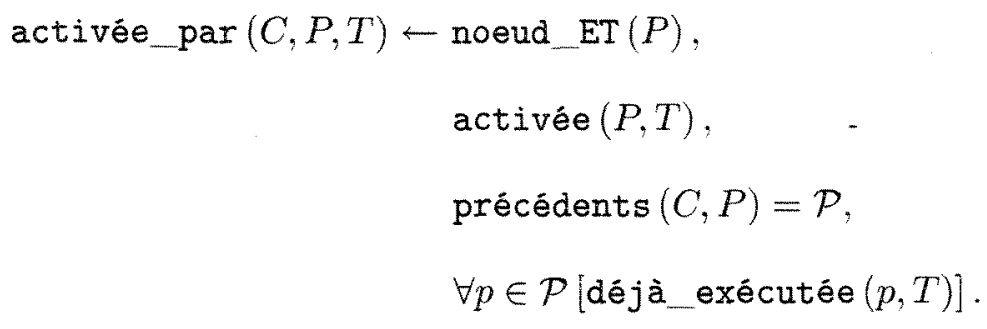


L'ensemble des prédécesseurs d'un nœud donné dans un plan est défini dans la librairie de plans. Une action primitive $A$ est précédemment exécutée au temps $T$ si et seulement si produit $\left(A, T^{\prime}\right)$ et $T^{\prime} \leq T$. C'est plus complexe pour les actions composites. La règle 2.12 donne les conditions pour les méthodes et la règle 2.13 exprime les conditions pour les buts :

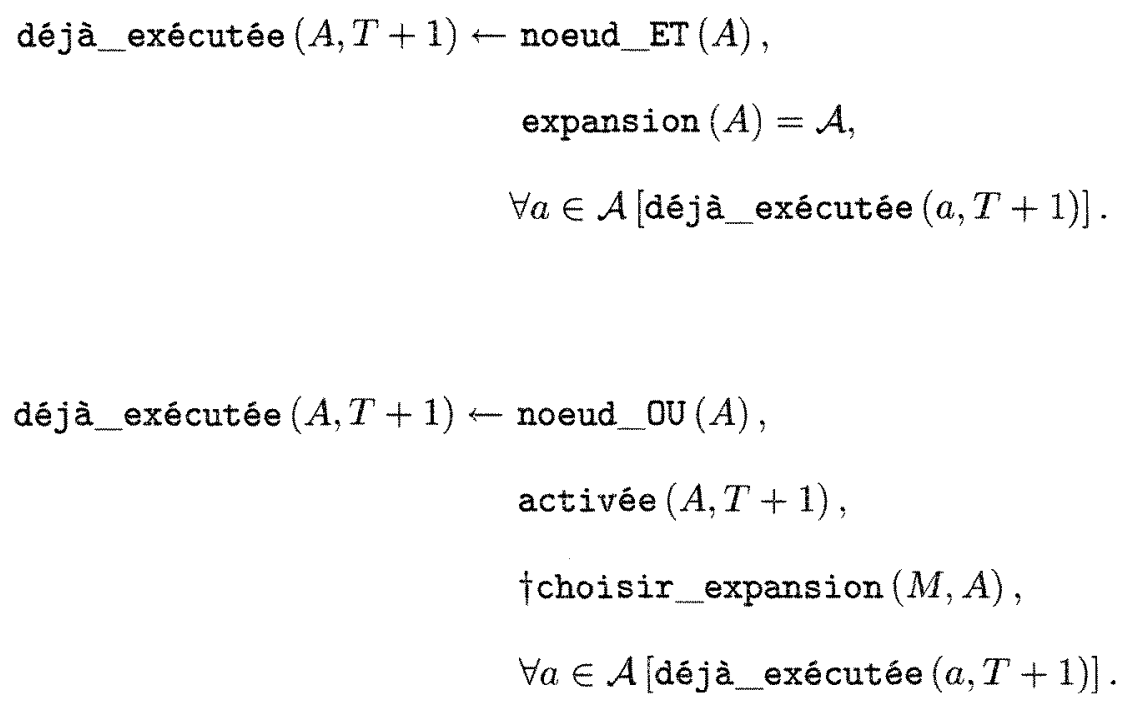

La deuxième façon pour qu'un enfant $C$ peut être activé en tant qu'élément d'un plan $P$ est que $C$ soit l'une des méthodes qui effectuent $P$. Dans ce cas, l'agent choisit $C$ comme étant la méthode qu'il utilisera pour réaliser $P$ :

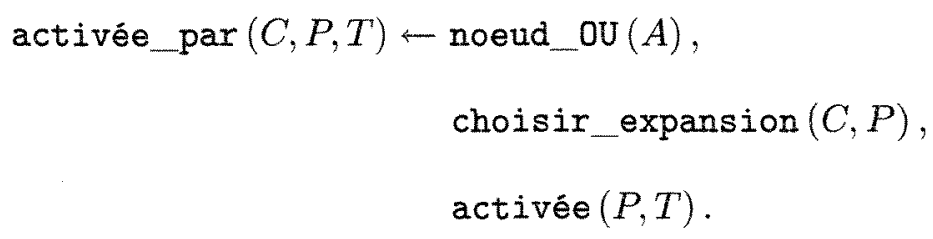

Les expressions de la forme choisir_expansion $(C, P)$ sont des hypothèses. Les méthodes d'un parent $P$ peuvent ne pas être équiprobables et on peut alors spécifier leurs probabilités $a$ priori. On suppose que l'agent choisit une seule méthode comme moyen d'accomplir $P$. 


\subsubsection{Progression de l'exécution}

Jusqu'à présent, les règles présentées expliquent le contenu initial de l'ensemble des actions en suspens et, par conséquent, permettent d'interpréter la première action effectuée par l'agent observé. Quand l'exécution des plans progresse, le contenu de cet ensemble change, tel qu'illustré sur la Figure 2.9 par les flèches ombragées :

$$
\begin{aligned}
\text { suspens }\left(P_{t+1}, T+1\right) \leftarrow & \operatorname{suspens}\left(P_{t}, T\right) \\
& \text { feuilles }(L s), \\
& \text { progression }\left(L s, P_{t}, P_{t+1}, T+1\right) .
\end{aligned}
$$

La règle progression assemble une nouvelle liste en suspens $P_{t+1}$ en considérant l'ensemble des actions primitives, ajoutant et enlevant les actions appropriées. Cette règle implique plusieurs cas :

1. l'action la plus récente est enlevée de l'ensemble en suspens;

2. toutes les actions précédemment en suspens restent en suspens;

3. les actions qui n'étaient pas précédemment en suspens peuvent être ajoutées.

Parce que les règles doivent être mutuellement exclusives et exhaustives, les règles pour la progression sont encombrantes et on donc été omises ici. Dans le cas de l'ajout d'actions primitives dans l'ensemble en suspens, ces actions doivent être activées et ne doivent pas avoir déjà été exécutées:

$$
\begin{aligned}
\text { ajouter_moi }(A, T) \leftarrow & \neg \text { déjà_exécutée }(A, T), \\
& \text { activée }(A, T) .
\end{aligned}
$$

\subsubsection{Incorporation du contexte}

Jusqu'à présent, on supposait que tous les plans avaient une probabilité a priori fixée d'être choisis. Le modèle d'exécution de plans peut être amélioré en ajoutant des règles qui 
permettent de rendre la sélection d'un plan conditionnelle à certaines propriétés de l'état de l'environnement lors du démarrage du processus de reconnaissance.

\subsubsection{Interaction avec l'agent observé}

Il est possible qu'un observateur souhaite intervenir dans le processus d'exécution de plans de l'agent observé afin de fournir de l'assistance à cet agent. Pour pouvoir prendre en compte l'intervention d'un observateur, il faut réviser le modèle pour prendre en compte les deux explications sur l'exécution d'une action :

1. l'agent observé effectue l'action;

2. le système intervient pour exécuter l'action.

On doit remplacer la règle 2.5 par les deux règles suivantes :

$$
\begin{aligned}
\text { produit }(X, T+1) \leftarrow & \text { intervenir }(X, T+1) . \\
\text { produit }(X, T+1) \leftarrow & \neg \text { intervenir }(X, T+1), \\
& \text { suspens }(P, T), \\
& X \in P, \\
& \text { sélectionné }(X, P, T+1) .
\end{aligned}
$$

La règle 2.18 est une révision de la règle originale 2.5 permettant de la rendre mutuellement exclusive et exhaustive avec la règle 2.17. La règle 2.17 indique qu'une intervention rend l'occurrence d'une action $X$ au temps $T+1$ indépendante des évènements précédents et du contenu de l'ensemble en suspens. Toutefois, cette règle d'intervention possède des effets de causalité sur le contenu futur de l'ensemble en suspens et donc, sur les prochaines actions effectuées par l'agent observé. 


\subsubsection{Le modèle probabilisé d'exécution de plans}

Afin d'ajouter un volet probabiliste à ce modèle logique, on doit spécifier les probabilités d'un ensemble restreint d'hypothèses. Ces probabilités sont les suivantes:

1. La probabilité que l'agent adopte une tâche particulière auto-motivée (pour elle-même). Ces probabilités peuvent être conditionnelles à l'état de l'environnement.

2. La probabilité que l'agent choisisse une méthode particulière en tentant d'atteindre un but.

3. La probabilité que l'agent choisisse une action primitive particulière à partir de l'ensemble des actions primitives en suspens.

Ces trois paramètres correspondent aux trois types d'hypothèses marqués par un $\dagger$.

On utilise ces trois critères pour calculer la probabilité d'une explication exp, moyennant un ensemble d'observations obs $[37,39]$, en se basant sur la formule de Bayes:

$$
P(\exp \mid o b s)=\frac{P(\exp \wedge o b s)}{P(o b s)}
$$

On peut aussi utiliser la formulation équivalente :

$$
P(\exp \mid o b s)=\frac{P(\exp \wedge \text { obs })}{\sum_{i} P(\exp \wedge \text { ^obs })}
$$

Afin de calculer la probabilité jointe d'une explication particulière et d'un ensemble d'observation, on factorise $P(o b s \mid e x p)$ en trois probabilités :

1. La probabilité a priori de chacun des nœuds-OU (les buts) $G_{i}$ que peut prendre l'agent. Cette probabilité est $P\left(G_{i}\right)$.

2. La probabilité a priori de chacun des nouds-ET (méthodes) $C h_{j}$ d'un but $j$. On suppose que chacune des méthodes est équiprobable pour un parent donné. Donc, si la taille des enfants d'un nœud-OU $j$ est $\left|C h_{j}\right|$, alors la probabilité de chacune des méthodes de ce but est $1 /\left|C h_{j}\right|$. 
3. La probabilité a priori de chacune des actions primitives de l'ensemble en suspens $P S_{k}$ pour être la prochaine à s'exécuter. On suppose que les actions de l'ensemble en suspens sont équiprobables et donc, la probabilité d'une action de l'ensemble est $1 /\left|P S_{k}\right|$.

On obtient donc la formule suivante :

$$
P(\exp , o b s)=\prod_{i=0}^{I} P\left(G_{i}\right) \prod_{j=0}^{J}\left(1 /\left|C h_{j}\right|\right) \prod_{k=0}^{K}\left(1 /\left|P S_{k}\right|\right)
$$

où le premier terme correspond à la probabilité de l'ensemble des buts de l'agent, le second terme désigne la probabilité que l'agent choisisse des plans particuliers pour compléter les buts, et le dernier terme est la probabilité que les observations données s'exécutent dans l'ordre spécifique d'exécution.

Pour trouver la probabilité qu'un agent a un but $G_{i}$ particulier, sachant un ensemble d'observations, on additionne les probabilités de toutes les explications contenant $G_{i}$, dénotées par $\operatorname{Exp}_{G_{i}}$. On a donc :

$$
P\left(G_{i} \mid o b s\right)=\frac{\sum_{e}^{E_{G_{i}}} P(e, o b s)}{\sum_{\varepsilon}^{\operatorname{Exp}} P(e, o b s)}
$$

où le dénominateur additionne la probabilité de toutes les explications des observations et le numérateur additionne la probabilité des explications dans lesquelles le but $G_{i}$ est présent.

\subsubsection{Gestion de l'observabilité partielle}

Le modèle d'exécution de plans permet de traiter le cas où l'environnement est partiellement observable $[38,39]$. Une façon simple de traiter ce cas dans le modèle est de quantifier la probabilité d'exécution d'une action par l'agent mais sans avoir été en mesure de l'observer. La probabilité de la non-observation, sachant une action donnée, est donc $P(\neg o b s(a) \mid a)$ ou $P\left(N o n o b s_{a}\right)$. Ensuite, on ajoute cette probabilité dans la formule 2.21 pour calculer la probabilité d'une explication donnée :

$$
P(\exp , o b s)=\prod_{i=0}^{I} P\left(G_{i}\right) \prod_{j=0}^{J}\left(1 /\left|C h_{j}\right|\right) \prod_{k=0}^{K}\left(1 /\left|P S_{k}\right|\right) \prod_{l=0}^{L} P\left(\text { Nonobs }_{a}\right)
$$


Maintenant qu'on tient compte du fait qu'une action puisse être exécutée même si elle n'est pas observée, on doit modifier l'algorithme de génération d'hypothèses. L'ensemble des actions en suspens doit alors contenir aussi les actions non observées. On doit alors prendre en considération toutes les actions qui contribuent à l'ensemble des buts de l'agent. On doit donc, lors de la génération des explications, considérer les deux cas suivants :

1. il est possible que chaque action, dans l'ensemble en suspens, soit exécutée ensuite et non observée.

2. les explications générées ne contiennent pas d'actions non observées (algorithme original). On a donc une expansion significative de l'espace de reconnaissance. Pour régler ce problème, on peut écarter les explications selon un seuil sur la valeur de la probabilité de l'échec d'observation :

$$
\prod_{l=0}^{L} P\left(\text { Nonobs }_{a}\right)<\text { Seuil }
$$

La valeur du seuil peut être déterminée par l'usager du système.

\subsubsection{Exemple de reconnaissance de plans}

Afin de mieux comprendre comment le modèle d'exécution de plans effectue le processus de reconnaissance, nous allons illustrer celui-ci à l'aide d'un exemple. Pour des fins de simplification, on suppose que tous les buts et les méthodes sont respectivement équiprobables. Dans cet exemple, on utilise la librairie de plans de la Figure 2.8. Conséquemment, $P\left(\right.$ intention(AugmenterPuissance) $\mid$ Ouvrir $P 1_{1}$ ) exprime la croyance postérieure du système voulant que l'agent ait l'intention d'augmenter la puissance en sachant que l'ouverture du panneau 1 s'était produite au temps $t$ (OuvrirP1 $1_{1}$ équivaut à produit(OuvrirP1, 1 )). On peut représenter l'ensemble des observations par $\omega$.

Si l'on considère que l'agent exécute l'action DuvrirP1 puis l'action VérifierTempérature, le système peut conclure que l'agent doit poursuivre AugmenterTempérature et, au

minimum, l'un des deux plans suivants : AugmenterPuissance et AugmenterNiveauO 2 . Le 
Tableau 2.1 présente les probabilités d'intentions de ces trois plans, sachant que les actions DuvrirP1 et VérifierTempérature sont observées.

\begin{tabular}{c|c} 
Plan & $P($ intention $(\mathrm{Plan} \mid \omega))$ \\
\hline AugmenterTempérature & 1.0 \\
AugmenterPuissance & 0.6603 \\
AugmenterNiveauO $_{2}$ & 0.6603
\end{tabular}

Tableau 2.1 - Probabilités d'intention des plans de l'exemple sachant les observations selon Goldman et al. [44]

Les probabilités d'intention ont été calculées à partir des probabilités a priori définies dans la librairie de plans et des observations. Étant donné que le plan AugmenterTempérature est le seul contenant l'action VérifierTempérature, sa probabilité d'intention est de 1 . Pour les plans AugmenterPuissance et AugmenterNiveauO $\mathrm{N}_{2}$, il s'agit des deux seuls plans contenant l'action OuvrirP1 et sont donc équiprobables entre eux, car la probabilité a priori de sélection des différents plans par l'agent est uniforme dans cet exemple.

La croyance du système à propos des buts multiples de l'agent lui permet d'assigner des probabilités plus élevées aux actions qui contribuent aux plans possibles de l'agent. Par exemple, DémarrerGénérateurB et AugmenterTempératureFixée constituent les prochaines étapes des plans respectifs. Conséquemment, le système anticipera que l'une des ces actions sera la prochaine exécutée:

$$
\begin{aligned}
P\left(\text { DémarrerGénérateurB }_{3} \mid \omega\right) & =0.4748 \\
P\left(\text { AugmenterTempératureFixée }_{3} \mid \omega\right) & =0.4748
\end{aligned}
$$

\subsubsection{Bilan sur les travaux de Geib et al.}

Les travaux de Geib et al. s'appuient sur une logique d'abduction probabiliste pour inférer les hypothèses qui expliquent une série d'actions observées et pour mieux cerner l'explication. Il y a deux principaux problèmes dans cette approche liés au fait que l'agent sélectionne initialement ses plans pour atteindre ses différents buts. Premièrement, il faut que l'agent observateur connaisse les intentions de l'agent observé pour pouvoir utiliser le modèle 
d'exécution des plans. Ceci n'est pas réaliste dans un contexte réel où l'agent observateur ne connaît pas les intentions de l'agent observé, et il doit alors considérer tous les plans de la librairie. Deuxièmement, étant donné que l'on suppose que l'agent observé sélectionne dès le départ ses plans à exécuter, on ne peut pas tenir compte du fait que l'agent puisse réagir dynamiquement aux changements qui peuvent survenir dans l'environnement. Le processus de reconnaissance de plans peut tenir compte de l'absence d'observation d'une action effectuée en ajoutant une probabilité de non-observation d'une action exécutée. On peut alors considérer les actions exécutées non observées, mais cela fait augmenter l'espace de reconnaissance (qu'on peut contrôler avec une valeur seuil). Il est difficile d'évaluer la probabilité de non-observation d'une action, car elle dépend de l'état du matériel du système, qui n'est pas constant. Aussi, on considère que l'agent observé est rationnel et donc, on ne peut pas considérer l'exécution erronée des plans.

\subsection{Conclusion}

Ce chapitre avait pour but de présenter un état de l'art sur les processus de reconnaissance de plans, plus particulièrement sur les approches hybrides. On a pu constater qu'il existe trois catégories d'approches de reconnaissance de plans : les approches logiques, probabilistes et hybrides. De plus, nous avons pu dégager deux types d'approches dans les processus de reconnaissance hybrides. Dans le premier type, tel que les travaux de Avrahami et al., on utilise un algorithme symbolique pour générer un ensemble d'hypothèses en fonction des observations, puis on utilise un modèle probabiliste pour désambiguïser ces hypothèses. L'utilisation d'un algorithme symbolique permet d'augmenter l'efficacité du système, car le modèle probabiliste travaille seulement sur un sous-ensemble de la librairie de plans au lieu de la totalité des plans possibles. Dans la deuxième approche hybride présentée, celle décrite par les travaux de Geib et al., on utilise une logique probabiliste pour inférer et quantifier les hypothèses. Donc, le processus d'inférence s'occupe aussi de la partie probabiliste. Les deux approches

hybrides présentent l'avantage de tenir compte des plans entrecroisés et de l'observabilité partielle des actions exécutées. Par conséquent, l'utilisation d'une méthode hybride permet de 
trouver des réponses au problème d'équiprobabilité des hypothèses inhérent aux approches logiques ainsi qu'au problème de la complexité du calcul des approches probabiliste. Toutefois, les approches hybrides présentées ici considèrent que l'agent observé est rationnel, c'est-à-dire que les exécutions erronées des plans ne sont pas prises en compte. De plus, on considère que la librairie de plans est complète et les hypothèses pouvant être générées y sont déjà incluses.

Le prochain chapitre présente un modèle hybride de reconnaissance qui tient compte du dilemme cité précédemment en permettant la génération dynamique d'hypothèses absentes de la librairie de plans. Ce modèle s'inscrit dans la lignée des travaux de Avrahami et al. en étendant un modèle logique existant avec une couche probabiliste. Le modèle logique servira de premier filtre pour générer des hypothèses consistantes et la couche probabiliste permettra de raffiner cet ensemble d'hypothèses en attribuant une valeur quantitative aux plans possibles, permettant ainsi de favoriser certaines hypothèses au détriment des autres. 


\section{Chapitre 3}

\section{UN MODĖLE HYBRIDE DE RECONNAISSANCE DE PLANS}

\subsection{Introduction}

Le modèle hybride de reconnaissance de plans proposé [81] peut être vu comme étant une extension probabiliste du modèle logique présenté dans les travaux de Bouchard et al. [21,22]. Ce modèle est basé sur la théorie des treillis et sur un cadre d'action formel basé sur la logique de description. L'intérêt de ce modèle logique est qu'il permet de transformer la problématique de la reconnaissance de plans en un problème de classification basé sur la relation de subsomption de la logique de description. Cette relation exploite le paradigme de classification pour faire correspondre un évènement observé avec le type d'action qui lui est rattaché. Les plans possibles identifiés par ce processus de correspondance sont ensuite organisés à l'intérieur d'une structure de treillis qui rend explicites les relations incertaines (partielles) entre les plans possibles, lesquels ne sont pas nécessairement directement comparables. On peut donc dynamiquement générer un ensemble d'hypothèses concernant les comportements entrecroisés ou erronés plausibles, lesquelles peuvent être induites par le déficit cognitif d'un patient. Cependant, ce modèle logique souffre d'une problématique d'équiprobabilité. En effet, on ne peut pas privilégier certaines hypothèses parmi l'ensemble des différentes hypothèses générées par ce processus logique. Pour pallier à cette limitation, on propose dans ce chapitre une extension probabiliste du modèle logique, laquelle est basée sur certains aspects des travaux portant sur les logiques de description probabilistes. Ce nouveau processus hybride permet de raffiner l'ensemble des hypothèses générées par le processus logique en utilisant les probabilités comme moyen quantitatif pour privilégier certaines hypothèses. 
La première partie de ce chapitre présentera un aperçu du modèle logique qui constitue le noyau sur lequel on appliquera l'extension probabiliste pour former un modèle hybride de reconnaissance de plans. Cette partie présentera les principaux éléments du modèle d'actions basé sur la logique de description qui constitue le fondement du modèle logique. Ensuite, on présentera les principaux éléments du modèle logique basé sur le modèle d'action et sur la théorie des treillis. La deuxième partie sera consacrée à la description du modèle hybride proposé, résultant de l'extension probabiliste du modèle logique.

\subsection{Aperçu du modèle logique de reconnaissance de plans}

Le but de cette section est de présenter un aperçu du modèle logique de reconnaissance de plans, détaillé dans les travaux de Bouchard et al. [21,22], qui subira une extension probabiliste pour obtenir le modèle hybride proposé. Le modèle logique de reconnaissance de plans $\mathcal{M}=\left\langle\mathcal{A}, \circ, \prec_{p}, D i s U, \oplus, \nabla, \Delta\right\rangle$ permet de représenter la base de connaissances d'un agent observateur, dans un contexte d'assistance. L'ensemble $\mathcal{A}=\left\{a_{1}, \cdots, a_{i}\right\}$ représente la totalité des actions que le patient peut réaliser et qui sont connues de l'agent observateur. On utilise l'opérateur de séquence o pour ordonner les actions incluses dans $\mathcal{A}$, dans le but de former un ensemble de structures de plans. Cet ensemble de plans constitue la librairie de plans (activités de la vie quotidienne) connus de l'agent observateur. On organise ces plans en une structure taxonomique à l'aide de la relation de subsomption $\prec_{p}$ définie entre les plans. Cette relation $\prec_{p}$ est une extension de la relation de subsomption définie à l'intérieur de la logique de description [6].

Le processus de reconnaissance logique est basé sur quatre opérateurs :

- l'opérateur de disunification $D i s U$,

- l'opérateur de composition $\oplus$,

- l'opérateur $\nabla$ calculant la borne supérieure entre deux plans,

- et l'opérateur $\Delta$ calculant la borne inférieure entre deux plans.

Ces opérateurs permettent la formation d'un cadre algébrique permettant de minimiser l'incertitude reliée au processus de reconnaissance de plans, en fournissant un moyen d'identifier 
efficacement l'ensemble des hypothèses plausibles pouvant expliquer le comportement d'un patient observé. Le point suivant présente un aperçu du modèle d'action [23,24] qui sert de fondement théorique pour le modèle logique.

\subsubsection{Le modèle d'action}

L'approche proposée pour la formalisation de l'action en logique de description suit les traces de différents travaux d'incorporation de la notion d'action dans les logiques de description $[9,56]$. La proposition consiste en un cadre formel de l'action basé sur le modèle état-transition, lequel peut être considéré comme une extension dynamique de la logique de description $[23,24]$. L'intérêt de ce modèle d'action est qu'il permet de transformer la problématique de reconnaissance de plans en un problème de classification. Ce modèle permet, en utilisant la relation de subsomption de plans, de raisonner sur les plans à différents niveaux d'abstraction. Donc, il permet à un agent de reconnaissance de plans de raisonner plus efficacement à propos des actions pouvant constituer des relations entre les différents plans $[2,64]$.

\subsubsection{1 Éléments de la logique de description}

Les logiques de description sont une famille de langages de représentation de connaissances qui peuvent être utilisés pour représenter la connaissance d'un domaine d'application d'une façon structurée et formelle [7]. Par conséquent, les différentes notions du domaine sont décrites à l'aide de descriptions de concept, c'est-à-dire des expressions qui sont construites à partir de concepts atomiques (prédicats unaires) et de rôles atomiques (prédicats binaires), qui mettent en relation les concepts, en utilisant les constructeurs de concepts et de rôles fournis par une logique de description particulière. En plus du formalisme de description, les logiques de description sont normalement équipées d'un formalisme terminologique et d'un formalisme assertionnel. Les axiomes terminologiques permettent de définir les concepts et les rôles, et les axiomes assertionnels permettent de fixer les propriétés des individus. Les logiques de description fournissent plusieurs processus d'inférence pour déduire la connaissance 
implicite à partir de la connaissance explicite représentée : la subsomption, l'appartenance, et la consistance.

Définition 3.1 (La subsomption) Un concept $C$ est subsumé par un concept $D$ si et seulement si toutes les instances de $C$ sont nécessairement des instances de $D$. En d'autres termes, la description (l'ensemble des individus) du concept $C$ est un sous-ensemble de la description du concept $D$.

Définition 3.2 (L'appartenance) Un individu $i$ est une instance de la description du concept $C$ si et seulement si $i$ est interprété comme étant un élément de $C$.

Définition 3.3 (La consistance) Une base de connaissances, constituée d'un ensemble d'assertions et d'un ensemble d'axiomes terminologiques, est consistante si et seulement si elle n'est pas contradictoire.

\subsubsection{Formalisation de l'action en logique de description}

On peut définir un modèle état-transition par une paire $(\mathcal{W}, \mathcal{A})$, où $\mathcal{W}$ représente l'ensemble des différents états possibles du monde, et $\mathcal{A}$ représente l'ensemble des actions qui sont applicables sur ces différents états.

Définition 3.4 (Action) Une action $a \in \mathcal{A}$ sur l'ensemble $\mathcal{W}$ est définie comme étant une relation binaire $a \subseteq \mathcal{W} \times \mathcal{W}$, tel que $a(w)=\{e \mid(w, e) \in \mathcal{W} \times \mathcal{W}\}$, où $w$ et e représentent, respectivement, l'état courant et l'état suivant.

À l'intérieur de ce cadre, les actions sont déterministes et opèrent sur les formules d'assertions, lesquelles sont des cas particuliers de formules de la logique du premier ordre [20]. Si les expressions conceptuelles et les assertions en logique de description sont utilisées pour décrire des faits à propos d'un état du monde (l'environnement du patient), elles peuvent être satisfiables ou insatisfiables selon cet état. Conséquemment, les états du monde peuvent correspondre à des structures sémantiques. Soit une structure sémantique $w=\left\langle\operatorname{Dom}(w),\left(\cdot{ }^{I_{w}}\right)\right\rangle$ de sorte que $\operatorname{Dom}(w)$ représente le domaine d'interprétation, c'est-à-dire l'ensemble non vide d'objets appelés individus qui existent dans le monde quand celui-ci est dans l'état $w$ à un 
moment spécifique. La fonction $\left({ }^{I_{w}}\right)$ réfère à une fonction d'interprétation associée à $w$, et assigne à chaque symbole de concept $C$ un sous-ensemble du domaine $\operatorname{Dom}(w)$, c'est-à-dire $C^{I_{w}} \in \operatorname{Dom}(w)$, et à chaque rôle un sous-ensemble du domaine $\operatorname{Dom}(w) \times \operatorname{Dom}(w)$, c'est-à-dire $r^{I_{w}} \in \operatorname{Dom}(w) \times \operatorname{Dom}(w)$, de sorte que les formules définissant la sémantique de la logique de description soient satisfaites [10].

Soit les symboles $C$ et $D$, qui désignent des noms de concept, et soit $r$, qui désigne le nom d'un rôle selon le sens de la logique de description. La relation de subsomption entre les objets de concept est dénotée par l'expression $C$ subsume $D$, ou $D \prec C$, laquelle est équivalente à $D^{I_{w}} \subseteq C^{I_{w}}$ dans l'état $w$. Une assertion de la forme $C(i)$ stipule que l'individu $i$ est une instance du concept $C$, et une assertion de la forme $r(i, j)$ indique que le couple d'individus $(i, j)$ est dans l'extension de $r$.

Les actions interviennent au niveau factuel (assertionnel) relatif à l'extension des concepts et rôles. Afin d'associer une interprétation aux assertions, la fonction $\left(. I_{w}\right)$ est étendue aux individus de sorte que, par exemple, $C(i)$ est satisfait par $w$. On note ainsi $w \vDash C(i)$ si et seulement si $i \in C^{I_{w}}$.

\subsubsection{Sémantique de la structure d'action en logique de description}

Une action $a(w)$ est structurée en une formulation inspirée de celle proposée par le langage STRIPS [35] et utilise ainsi les notions de préconditions et de postconditions.

Définition 3.5 (Préconditions) L'ensemble des états dans lesquels on peut exécuter l'action $a(w)$ correspond au domaine $\operatorname{Dom}(a)$, où $\operatorname{Dom}(a)=\{w \in \mathcal{W} \mid w \models$ pre $(a)\}$. La précondition pre(a) de l'action a consiste en une conjonction de formules d'assertions concernant les objets conceptuels ainsi que les rôles qui lient ces objets. Par conséquent, toutes les assertions composant la précondition pre $(a)$ doivent être satisfaites par chaque état $w$, de sorte que $a(w) \neq \emptyset$.

Définition 3.6 (Postconditions) L'ensemble des états résultant de l'exécution de l'action $a$ correspond au co-domaine $\operatorname{CoDom}(a)=\{e \in \mathcal{W} \mid e \vDash p o s(a)\}$. La postconditon pos $(a)$ représente les effets produits par l'exécution de l'action $a(w)$, qui peut être exprimée par 
les formules d'assertion $\operatorname{pos}^{+}(a)$ et $\operatorname{pos}^{-}(a)$ qui change l'interprétation des concepts et rôles impliqués dans une action $a(w)$. La formule $\operatorname{pos}^{+}(a)$ décrit les ajouts à l'interprétation des concepts et rôles, et la formule $\operatorname{pos}^{-}(a)$ décrit, inversement, les suppressions à l'interprétation des concepts ou rôles.

\subsubsection{La relation de subsomption entre les actions}

Maintenant, on peut définir la relation de subsomption qui organise les concepts d'actions en une taxonomie.

Définition 3.7 (Subsomption d'actions) Soit $a$ et $b$, deux structures d'actions construites selon le modèle présenté, il existe une relation de subsomption entre ces deux actions, où $b$ est le subsumant et a le subsumé, si et seulement si le domaine et le co-domaine de l'action $a$ sont respectivement inclus dans le domaine et le co-domaine de l'action $b$. Formellement, une action $b$ subsume une action $a$, dénoté par $a \prec_{a} b$, si et seulement si $\operatorname{Dom}(a) \subseteq \operatorname{Dom}(b)$ et $\operatorname{CoDom}(a) \subseteq \operatorname{CoDom}(b)$.

La sémantique de cette relation de subsomption d'actions est une extension directe de la relation de subsomption traditionnelle provenant de la logique de description et constitue l'élément de base sur lequel s'appuie le processus de reconnaissance de plans défini dans la suite de ce chapitre. On peut formellement exprimer cette relation de subsombtion d'action par :

$$
\begin{aligned}
b \prec_{a} a \rightarrow & \forall(w, e) \\
& (w \models \operatorname{pre}(b) \rightarrow w \models \operatorname{pre}(a)) \wedge \\
& \left(w \models \operatorname{pos}^{\dagger}(b) \rightarrow w \models \operatorname{pos}^{+}(a)\right) \vee \\
& \left(w \models \operatorname{pos}^{-}(b) \rightarrow w \models \operatorname{pos}^{-}(a)\right) .
\end{aligned}
$$




\subsubsection{Sémantique d'un plan d'actions en logique de description}

À partir de ce modèle d'action en logique de description, on définit la notion de structure de plan d'actions qui sera utilisée pour décrire les différentes activités de la vie quotidienne réalisée par le patient.

Définition 3.8 (Plan d'actions) Un plan d'actions $\alpha$ consiste en une séquence d'actions $\left(a_{1}, \ldots, a_{n}\right)$, dénoté $\alpha\left(a_{n} \circ a_{n-1} \circ \cdots \circ a_{1}\right)$, qui permet d'effectuer la transition d'un état initial $w_{0}$ vers un état final $w_{n}$ de l'environnement, en passant par une succession d'états $w_{i}$, où $i \in[1, n]$.

Le symbole o est un opérateur de séquence qui caractérise la composition de deux actions, c'est-à-dire qu'une certaine action est appliquée sur l'état résultant de l'exécution de l'action précédente, de sorte que $\left(a_{2} \circ a_{1}\right)\left(w_{0}\right)=a_{2}\left(a_{1}\left(w_{0}\right)\right)=w_{2}$. On peut noter que si $a_{1}: \mathcal{W} \rightarrow \mathcal{W}$ et que $a_{2}: \mathcal{W} \rightarrow \mathcal{W}$, alors $\left(a_{2} \circ a_{1}\right): \mathcal{W} \rightarrow \mathcal{W}$, où $\mathcal{W}$ est l'ensemble des états possibles de l'environnement. En d'autres termes, un plan est un ensemble d'actions totalement ordonné $\left(\bigcup_{i=1, n}\left\{a_{i}\right\}, 0\right)$. On peut donc maintenant interpréter formellement l'exécution d'un plan d'actions $\alpha\left(a_{n} \circ a_{n-1} \circ \cdots \circ a_{1}\right)$ à partir d'un état $w$ de l'environnement à l'aide de :

$$
\alpha(w)=a_{n}\left(a_{n-1}\left(\ldots\left(a_{1}(w)\right) \ldots\right)\right)
$$

La sémantique lié à la notion de plan est décrite par la composition des fonctions d'interprétations individuelles, qui spécifie l'évolution de l'interprétation des concepts et des rôles à travers du domaine lors de l'exécution distincte de chacune des actions impliquées dans le plan d'actions.

En utilisant cette structure de plan d'actions en logique de description, on peut maintenant décrire la librairie de l'agent observateur, qui consiste à l'ensemble des activités que l'agent observé peut effectuer dans l'environnement et qui sont connues par l'agent observateur. La prochaine section définit algébriquement le processus de reconnaissance de plans, et utilisera 
la structure d'action et la structure de plan d'actions pour déterminer les différents plans possiblement en cours d'exécution.

\subsubsection{Définition algébrique du processus de reconnaissance}

Dans cette section du chapitre, le cadre algébrique régissant le processus logique de reconnaissance d'activités de haut niveau à l'intérieur d'un habitant intelligent sera défini en se basant sur le modèle d'action en logique de description précédemment présenté.

\subsubsection{L'ensemble des plans possibles}

Dans notre contexte d'assistance cognitive à l'intérieur d'un habitat intelligent, le processus de reconnaissance d'activités consiste, pour l'agent observateur assistant, à déterminer l'ensemble minimal de plans possibles permettant d'interpréter la séquence d'actions effectuées par un patient atteint de la maladie d'Alzheimer. Cet ensemble de plans possibles permet de prédire les actions futures du patient et de caractériser l'ensemble de plans plausibles qui explique son comportement et anticipe les futures déviations du patient. Pour cela, on utilisera la librairie de plans connus de l'agent observateur pour extraire les différents plans possibles qui correspondent aux actions observées.

Définition 3.9 (Plan possible) Soit $\mathcal{A}$, l'ensemble des actions connues par l'agent observateur (la librairie d'actions), et soit $\mathcal{P}$, l'ensemble des plans connus de l'agent observateur (la librairie de plans). Soit $\mathcal{O}$, l'ensemble des actions observées par l'agent observateur, de sorte que $\forall 0 \in \mathcal{O}$, $\exists a \in \mathcal{A}$ où $a(o)$ est satisfait. L'assertion $a(o)$ signifie que l'action observée $o$ correspond à une instance du type d'action $a$. Un plan $\alpha$ est un plan possible pour une certaine action observée $a(o)$ si et seulement si $a \in \alpha$, c'est-à-dire si le plan d'actions $\alpha$ contient une composante de type $a$ à l'intérieur de sa séquence d'actions.

L'ensemble des plans possibles qui peuvent expliquer une certaine observation o est défini par :

$$
\mathcal{P}_{s}^{o}=\{\alpha \in \mathcal{P} \mid \exists a \in \alpha, a(o)\}
$$




\subsubsection{Gestion de la multiplicité des observations}

Il est tout simplement irréaliste de supposer que toutes les actions observées sont reliées à un seul et unique plan, car l'agent observé peut effectuer plusieurs plans en parallèle. Alors, on doit prendre en considération l'ensemble des plans possibles qui peuvent correspondre à chacune des actions observées individuelles, ce qui se traduit formellement par l'union des ensembles de plans pouvant expliquer chacune des observations, tel que défini par la formule suivante :

$$
\mathcal{P}_{S}^{\mathcal{O}}=\bigcup_{o_{i} \in \mathcal{O}} \mathcal{P}_{s}^{o_{i}}
$$

où $\mathcal{P}_{s}^{\mathcal{O}}$ représente l'ensemble des plans possibles pouvant expliquer l'ensemble des actions observées $\mathcal{O}$.

\subsubsection{Le schéma d'intentions}

Si l'on suppose que la librairie de plans (la base de connaissances) de l'agent observateur contient tous les plans cohérents que le patient (agent observé) peut effectuer, alors on peut supposer que le patient a l'intention, à travers ses actions, de réaliser l'un de ces plans cohérents. Néanmoins, le déficit cognitif du patient peut l'amener à agir de manière incohérente et son comportement peut alors dévier pour aller au-delà de l'ensemble des plans possibles provenant de la librairie de plans connus de l'agent observateur. Pour être en mesure d'expliquer ces anomalies comportementales plausibles du patient, on introduit une structure qui étend la notion de plan d'actions décrite précédemment : le schéma d'intentions. Cette structure nous permet de caractériser l'incertitude dans la prédiction des actions futures, en synthétisant l'ensemble des hypothèses plausibles pouvant être formulées en fonction du comportement antérieur du patient.

On peut définir le schéma d'intentions comme étant un plan contenant au moins une variable d'actions à l'intérieur de sa séquence. Les variables d'actions servent à caractériser l'incertitude reliée à chaque prédiction individuelle d'actions futures dans le comportement du patient. 
Définition 3.10 (Variable d'actions) Soit $\mathcal{V}$, un ensemble de variables d'actions. Une variable d'actions $x_{i} \in \mathcal{V}$, incluse à l'intérieur d'un plan $\alpha$, possède un domaine de substitution $S u b\left(x_{i}\right) \subseteq \mathcal{A}^{+}$, où $\mathcal{A}^{+}=(\wp(\mathcal{A}) \cup\{\varepsilon\}), \mathcal{A}$ est l'ensemble d'actions, et $\wp(\mathcal{A})$ est l'ensemble des parties de $\mathcal{A}$.

Le symbole $\varepsilon$ représente un type particulier d'action qui permet de caractériser la possibilité qu'un patient omette une action (une étape) de l'activité en cours de réalisation. Autrement dit, l'action $\varepsilon$ définit une transition, d'une certaine durée, pour laquelle l'état actuel de l'environnement du patient restera inchangé. Alors, l'interprétation des concepts et des rôles qui définissent l'état actuel ne sera pas modifiée lors de cette transition. Par conséquent, on peut qualifier $\varepsilon$ comme étant une action vide ou une absence d'action, ce qui est représentée formellement par $\varepsilon(w)=w$.

Définition 3.11 (Substitution) Une substitution $\sigma: \mathcal{V} \cup \mathcal{A} \mapsto \mathcal{A}^{+}$est définie de la façon suivante :

$$
\sigma(a)= \begin{cases}a & \text { si } a \in \mathcal{A} \\ e \in \operatorname{Sub}(a) & \text { si } a \in \mathcal{V}\end{cases}
$$

Cette procédure de substitution des variables d'actions permet d'instancier le schéma d'intentions, pour générer, en fonction des observations, l'ensemble des extra-plans plausibles qui ne sont pas déjà préétablis dans la librairie de plans de l'agent observateur.

Définition 3.12 (Schéma d'intentions) Le plan $\left(a_{n} \circ \cdots \circ x \circ \cdots a_{1}\right)$ est un schéma d'intentions s'il existe une substitution $\sigma$, de sorte que $\left(a_{n} \circ \cdots \circ \sigma(x) \circ \cdots \circ a_{1}\right)$ constitue un plan plausible (hypothèse).

Un plan obtenu à partir d'un schéma d'intentions est plausible si l'on peut le classifier à l'intérieur de l'espace de reconnaissance des plans possibles (provenant de la librairie de plans) ayant produit le schéma d'intentions. La présence de variables à l'intérieur d'un schéma d'intentions se produit lors du calcul de la borne inférieure entre deux plans possibles 
incomparables provenant de la librairie de plans. Deux plans sont incomparables lorsqu'il y a une $i^{e}$ position dans les deux séquences d'actions n'admettant pas de subsumé commun.

\subsubsection{La relation de subsomption entre les plans}

À partir des différentes notions introduites précédemment, on peut définir la relation de subsomption qui permet d'organiser les plans en une structure taxonomique.

Définition 3.13 (Subsomption de plans) Soit $\alpha$ et $\beta$, deux structures de plans d'actions. Le plan $\beta$ subsume le plan $\alpha$, dénoté par $\alpha \prec_{p} \beta$, s'il existe une substitution $\sigma$, de sorte que $\forall i \in[1,|\beta|], \exists j \in[1,|\alpha|],\left(a_{j}, b_{i}\right) \in \alpha \times \beta$ alors $\sigma\left(a_{j}\right) \prec_{a} \sigma\left(b_{i}\right)$, où $|\alpha|$ et $|\beta|$ représentent la cardinalité des plans $\alpha$ et $\beta$.

À partir de cette relation de subsomption de plans, laquelle ordonne partiellement les plans possibles, on organise les plans possibles en une structure de treillis, laquelle permettra d'expliciter les extra-plans implicites, c'est-à-dire ceux qui sont induits par les intra-dépendances entre les plans possibles, en appliquant les opérations de composition $\oplus^{1}$ et de disunification DisU $U^{2}$ sur chaque paire de plans possibles incomparables.

\subsubsection{5 Éléments de la théorie des treillis}

Afin d'améliorer la compréhension des notions qui seront présentées prochainement, voici quelques éléments de base sur la théorie des treillis [29,45].

Définition 3.14 (Ordre) Un ordre $\mathbf{A}=\langle A, \leq\rangle$ consiste en un ensemble non vide $A$ et une relation binaire $\leq$ sur $A(\leq \subseteq A \times A)$, appelée relation d'ordre, de sorte que la relation $\leq$ est réflexive $(a \leq a$, pour tout $a \in A)$, antisymétrique $(a \leq b$ et $b \leq a$ implique $a=b$, pour tout $a, b \in A$ ), et transitive ( $a \leq b$ et $b \leq c$ implique $a \leq c$, pour tout $a, b, c \in A$ ). Un ordre qui est linéaire ( $a \leq b$ ou $b \leq a$, pour tout $a, b \in A$ ) est une chaîne.

\footnotetext{
${ }^{1}$ Opération permettant d'obtenir l'ensemble des combinaisons plausibles (hypothèses) entre deux plans possibles (de la librairie) qui expliquent l'ensemble des actions observées.

${ }^{2}$ Opération entre deux actions qui permet d'obtenir une action qui les subsume.
} 
Définition 3.15 (Majorant et minorant) Soit un ordre $\mathbf{P}$ et un sous-ensemble $H \subseteq \mathbf{P}$. Un élément $u$ est un majorant de $H$ si et seulement si $h \leq u$, pour tout $h \in H$. Un élément $v$ est un minorant de $H$ si et seulement si $h \geq v$, pour tout $h \in H$.

Définition 3.16 (Bornes supérieure et inférieure) Un majorant $u$ de $H$ est la borne supérieure de $H$ si et seulement si, pour tout majorant $m$ de $H$, on a $u \leq m$. Un minorant $v$ de $H$ est la borne inférieure de $H$ si et seulement si, pour tout minorant $n$ de $H$, on a $v \geq n$.

On peut dénoter la borne supérieure de $H$ par $\bigvee H$ et la borne inférieure de $H$ par $\wedge H$. On utilise la notation $a \wedge b=\wedge\{a, b\}$ et $a \vee b=\vee\{a, b\}$, où $\wedge$ (ou $\Delta$ ) est la borne inférieure et $\vee($ ou $\nabla)$ est la borne supérieure des éléments $a$ et $b$.

Définition 3.17 (Treillis) Un ordre $\mathbf{L}$ est un treillis si et seulement si $a \wedge b$ et $a \vee b$ existent dans $L$ pour tout $a, b \in L(\wedge$ et $\vee$ sont des opérations binaires $L \times L \rightarrow L)$.

Les opérations binaires $\wedge$ et $\vee$ sont idempotentes $(a \wedge a=a$ et $a \vee a=a$, pour tout $a \in L)$, commutatives $(a \wedge b=b \wedge a$ et $a \vee b=b \vee a$, pour tout $a, b \in L)$, associatives $((a \wedge b) \wedge c=a \wedge(b \wedge c)$ et $(a \vee b) \vee c=a \vee(b \vee c)$, pour tout $a, b . c \in L)$, et satisfont les identités d'absorptions $(a \wedge(a \vee b)=a$ et $a \vee(a \wedge b)=a$, pour tout $a, b \in L)$.

\subsubsection{La composition des plans possibles}

On peut maintenant définir la notion de composition des plans possibles. Le but de l'opération de composition est de déterminer l'ensemble des combinaisons cohérentes entre les actions futures qui succèdent aux observations dans les plans possibles.

Définition 3.18 (Composition) Soit $\alpha, \beta \in \mathcal{P}_{s}^{\mathcal{O}}$, deux plans possibles permettant d'interpréter une séquence d'actions observées $\mathcal{O}$ à un certain temps $t$. La composition entre les plans $\alpha$ et $\beta$, dénotée par $\alpha \oplus \beta$, correspond à un ensemble d'extra-plans vérifiant les propriétés suivantes : stabilité et fermeture. 
Stabilité : Chaque extra-plan inclus dans $\alpha \oplus \beta$ est formé de :

1. d'au moins une action observée $o \in \mathcal{O}$,

2. d'au moins une action commune aux plans $\alpha$ et $\beta$,

3. une composition d'actions qui sont composantes de $\alpha$ ou $\beta$.

On note qu'il n'y a pas d'introduction d'actions externes à l'intérieur de l'extra-plan.

Fermeture : Chaque extra-plan inclus dans $\alpha \oplus \beta$ doit admettre une borne supérieure $\alpha \nabla \beta$ et une borne inférieure $\alpha \Delta \beta$. Ainsi, les extra-plans seront inclus dans l'intervalle $[\alpha \Delta \beta, \alpha \nabla \beta]$.

Cette opération de composition permet d'extraire, en fonction de l'ensemble des actions observées $\mathcal{O}$, tous les extra-plans plausibles anticipés qui sont susceptibles d'être réalisés par le patient. Conséquemment, cette opération de composition permet de compléter dynamiquement la base de connaissances de l'agent observateur en ajoutant ces extra-plans. Ces extra-plans peuvent être répartis en deux catégories. Dans la première catégorie, ces extra-plans représentent la combinaison de plusieurs plans connus qui sont exécutés de manière entrecroisée. Ces extraplans représentent alors un comportement cohérent du patient absent de la librairie de plans connus de l'agent observateur, dans lequel le patient possède plusieurs objectifs. Dans la seconde catégorie, les extra-plans représentent des déviations comportementales possibles du patient, pouvant résulter des symptômes de sa maladie.

Pour distinguer les deux catégories d'extra-plans, on utilise le critère de consistance qui permet de déterminer les extra-plans qui représentent un comportement cohérent du patient.

Consistance : Un extra-plan est consistant dans $\alpha \oplus \beta$ si et seulement s'il satisfait les propriétés de stabilité et de fermeture et qu'il est formé d'un ensemble de plans partiels, pour lequel chaque élément correspond à un plan inclus dans la base de connaissance $\mathcal{P}$ de l'agent observateur.

\subsubsection{L'espace de reconnaissance de plans}

À partir de la notion de la composition de plans, on peut définir la notion des plans plausibles. 
Définition 3.19 (Plans plausibles) L'ensemble des plans plausibles (ou hypothèses) $\mathcal{P}_{l}^{\mathcal{O}}$ consiste à l'union des paires de composition de plans possibles, lesquelles peuvent être générées à l'aide de l'instanciation du schéma minimal d'intentions correspondant à l'ensemble des observations $\mathcal{O}$, de sorte que :

$$
\mathcal{P}_{l}^{\mathcal{O}}=\bigcup_{\alpha, \beta \in \mathcal{P}_{s}^{\mathcal{O}}} \alpha \oplus \beta
$$

On note que $\mathcal{P}_{s}^{\mathcal{O}} \subseteq \mathcal{P}_{l}^{\mathcal{O}}$. Cet ensemble $\mathcal{P}_{l}^{\mathcal{O}}$ constitue un modèle d'interprétation valide pour l'ensemble des observations $\mathcal{O}$ s'il forme une structure de treillis à l'aide de la relation de subsomption de plans, et si chaque couple de plans incomparables dans $\mathcal{P}_{l}^{\mathcal{O}}$ admet une borne supérieure $\nabla$ et une borne inférieure $\Delta$ à l'intérieur de cet ensemble ordonné.

Théorème 3.1 (Espace de reconnaissance) L'ensemble des plans plausibles $\mathcal{P}_{l}^{\mathcal{O}}$, ordonné par la relation de subsomption de plans $\prec_{p}$, forme une structure de treillis, dénotée par $\Re_{o}=\left\langle\mathcal{P}_{l}^{\mathcal{O}}, \prec_{p}, \nabla, \Delta\right\rangle$, constituant ainsi un espace de reconnaissance.

Cet espace de reconnaissance représente un modèle d'interprétation du comportement du patient observé.

Définition 3.20 (Borne supérieure) Soit $\alpha\left(a_{n} \circ \cdots \circ a_{1}\right), \beta\left(b_{m} \circ \cdots \circ b_{1}\right) \in \mathcal{P}_{s}^{\mathcal{O}}$, deux plans qui interprètent les actions observées $\mathcal{O}$, où $|\mathcal{O}|=k$. La borne supérieure $\alpha \nabla \beta$ entre ces deux plans consiste au plan partiel commun le plus spécifique $\pi\left(c_{r} \circ \cdots \circ c_{1}\right)$ qui subsume $\alpha$ et $\beta$, de sorte que $\forall i \in[1, r]$, avec $k \leq r \leq \min (m, n), \forall o_{j} \in \mathcal{O}$, avec $j \in[1, k], \forall\left(a_{i}, b_{i}\right) \in \alpha \times \beta$, alors $c_{j}\left(o_{j}\right), a_{i} \prec_{p} \quad c_{i}$ et $b_{i} \prec_{p} c_{i}$.

Le symbole $\pi$ représente le résultat du calcul de la borne supérieure entre les deux plans possibles $\alpha$ et $\beta$. Le résultat minimal de l'opération $\alpha \nabla \beta$ consiste au plan partiel composé uniquement d'une action de type $\varepsilon$, laquelle subsume tous les plans. Ce plan partiel doit contenir toutes les actions observées qui sont communes aux deux plans.

La borne inférieure entre deux plans possibles incomparables représente une synthèse des observations et des prédictions pouvant être formulées à partir de ces observations. Le résultat 
contient donc toutes les actions observées $o \in \mathcal{O}$ et des variables d'actions, lesquelles permettent d'anticiper les actions futures du patient. Le calcul de cette borne permet donc d'obtenir un schéma minimal d'intentions en disunifiant les paires de plans possibles incomparables en utilisant l'opérateur de disunification DisU [49], issu de la logique du premier ordre. L'opération de disunification permet de trouver des relations entre des plans qui sont a priori incomparables, pour organiser et classifier ces plans à l'intérieur d'une structure de treillis. Le schéma d'intentions obtenu à partir du calcul de la borne inférieure est utilisé pour réunifier les plans possibles à travers l'opération de composition, ce qui permet de générer dynamiquement un ensemble d'hypothèses expliquant le comportement du patient.

Définition 3.21 (Borne inférieure) Soit $\alpha\left(a_{n} \circ \cdots \circ a_{1}\right), \beta\left(b_{m} \circ \cdots \circ b_{1}\right) \in \mathcal{P}_{s}^{\mathcal{O}}$, deux plans possibles interprétant les actions observées $\mathcal{O}$, où $|\mathcal{O}|=k$. La borne inférieure $\alpha \Delta \beta$ entre ces deux plans correspond au plan commun le plus général subsumé par ceux-ci, et est définie par : $\alpha \Delta \beta= \begin{cases}b_{m} \circ \ldots \circ b_{n+1} \circ \operatorname{Dis} U\left(a_{n}, b_{n}\right) \circ \ldots \circ \operatorname{DisU}\left(a_{k+1}, b_{k+1}\right) \circ \circ_{k} \circ \ldots \circ \circ_{1} & \text { si } n \leq m \\ a_{n} \circ \ldots \circ a_{m+1} \circ \operatorname{Dis} U\left(a_{m}, b_{m}\right) \circ \ldots \circ \operatorname{Dis} U\left(a_{k+1}, b_{k+1}\right) \circ o_{k} \circ \ldots \circ o_{1} & \text { si } m \leq n .\end{cases}$

Définition 3.22 (La disunification) L'opération de disunification $D i s U$ est injective $(\mathcal{A} \times \mathcal{A} \mapsto$ $\mathcal{A} \cup \mathcal{V} \cup\{\varepsilon\})$ sur l'ensemble des actions incomparables des plans $\alpha$ et $\beta$ :

$$
\operatorname{Dis} U(a, b)= \begin{cases}a & \text { si } a \prec_{a} b \\ b & \text { si } b \prec_{a} a \\ c & \text { si } \exists c \in \mathcal{A}: c \prec_{a} a \text { et } c \prec_{a} b \\ x & \text { sinon, avec } \operatorname{Sub}(x)=\{\varepsilon, a, b, a \circ b, b \circ a\}\end{cases}
$$

où $\mathcal{A}$ est l'ensemble des actions et $\mathcal{V}$ est l'ensemble des variables d'actions. 


\subsubsection{Exemple du processus de reconnaissance de plans}

Le présent exemple a pour but de faciliter la compréhension des différentes notions du modèle logique que nous venons de présenter. Supposons que l'agent observateur connaît les activités de la vie quotidienne CuirePâtes, PréparerThé et RegarderTélévision, où CuirePâtes est composée des étapes SaisirPâtes, FaireBouillirEau, et MettrePâtesDansEau, PréparerThé est composée des étapes PrendreTasse, FaireBouillirEau, et SaisirSachetThé, et RegarderTélévision est composée des étapes PrendreTélécommande, AllerSurDivan, et OuvrirTélévision. Ces trois activités constituent alors trois structures de plans qui sont dans la librairie de plans $\mathcal{P}$ de l'agent observateur, de sorte que $\mathcal{P}=\{$ CuirePâtes, PréparerThé, RegarderTélévision $\}$, où CuirePâtes(MettrePâtesDansEau o FaireBouillirEau ○ SaisirPâtes), PréparerThé(SaisirSachetThé

- FaireBouillirEau ○ PrendreTasse), et RegarderTélévision(OuvrirTélévision o AllerSurDivan - PrendreTélécommande). On note que (SaisirPâtes, FaireBouillirEau, MettrePâtesDansEau, PrendreTasse, SaisirSachetThé, PrendreTélécommande, AllerSurDivan, OuvrirTélévision $\} \subseteq \mathcal{A}$, où $\mathcal{A}$ est la librairie d'actions connues par l'agent observateur.

Si l'on suppose que la première observation est l'action SaisirPâtes, d'où SaisirPâtes $\left(o_{1}\right)$, alors pour l'ensemble des observations $\mathcal{O}=\left\{o_{1}\right\}$, l'ensemble des plans possibles est $\mathcal{P}_{s}^{\mathcal{O}}=\mathcal{P}_{s}^{o_{1}}=$ $\{$ CuirePâtes\}. Si l'on considère l'observation d'une deuxième action de type FaireBouillirEau, d'où FaireBouillirEau $\left(o_{2}\right)$, alors pour l'ensemble des observations $\mathcal{O}=\left\{o_{1}, o_{2}\right\}$, l'ensemble des plans possibles qui expliquent ces observations est $\mathcal{P}_{s}^{\mathcal{O}}=\mathcal{P}_{s}^{o_{1}} \cup \mathcal{P}_{s}^{o_{2}}=\{$ CuirePates, PréparerThé\}.

Reprenons maintenant l'exemple avec l'ensemble d'observations $\mathcal{O}=\{o\}$, avec FaireBouillirEau $(o)$ et $\mathcal{P}_{s}^{\mathcal{O}}=\mathcal{P}_{s}^{o}=\{$ CuirePates, Préparer Thé $\}$. On veut maintenant générer l'ensemble des plans plausibles (hypothèses) $\mathcal{P}_{l}^{\mathcal{O}}$ qui se retrouvent à l'intérieur de l'espace de reconnaissance $\Re_{o}$ à l'aide de l'opération de composition des plans possibles CuirePâtes $\oplus$ PréparerThé. Pour ce faire, on utilise la borne supérieure CuirePâtes $\nabla$ PréparerThé et la borne inférieure CuirePâtes $\triangle$ PréparerThé pour générer ces hypothèses expliquant le comportement du patient observé. La borne supérieure entre ces deux plans possibles est 
CuirePâtes $\nabla$ PréparerThé(FaireBouillirEau) et subsume CuirePâtes et PréparerThé. La borne inférieure entre ces deux plans possibles, qui est le schéma d'intentions du patient, est CuirePâtes $\Delta$ PréparerThé $(y \circ$ FaireBouillirEau $\circ x)$, où $S u b(x)=\{$ SaisirPâtes, PrendreTasse, SaisirPâtes $\circ$ PrendreTasse, PrendreTasse $\circ$ SaisirPâtes, $\varepsilon\}$ et $S u b(y)=\{$ MettrePâtesDansEau, SaisirSachetThé, MettrePâtesDansEau ○ SaisirSachetThé, SaisirSachetThé o MettrePâtesDansEau, $\varepsilon\}$.

À partir du schéma d'intentions, on génère dynamiquement les différentes hypothèses en substituant les variables avec les différentes valeurs de leur domaine de substitution, pour ainsi obtenir l'espace de reconnaissance $\Re_{0}=\{$ CuirePâtes, PréparerThé, CuirePâtes $\nabla$ PréparerThé, CuirePâtes $\Delta$ PréparerThé, $\left.H_{1}, H_{2}, \ldots\right\}$ tel que présenté par la Figure 3.1. Les différents extra-plans générés représentent des comportements erronés ou entrecroisés de la part du patient. Par exemple, l'extra-plan $H_{1}$ (SaisirSachetThé ○ FaireBouillirEau o SaisirPâtes) représente l'anticipation d'une erreur de type réalisation où le patient effectue une déviation de l'activité CuirePâtes vers l'activité PréparerThé. Aussi, l'extra-plan $\mathrm{H}_{2}$ (MettrePâtesDansEau o SaisirSachetThé o FaireBouillirEau ○ SaisirPâtes o Prendre Tasse) représente la possibilité que le patient réalise les activités CuirePâtes et PréparerThé de façon entrecroisée. Par conséquent, on remarque que le processus logique de reconnaissance de plans permet de circonscrire et borner le comportement potentiel du patient à l'intérieur d'un modèle d'interprétation structuré en treillis.

\subsubsection{Bilan du modèle logique}

En résumé, le processus logique de reconnaissance de plans, tel que synthétisé par la Figure 3.2, permet de dégager un espace de reconnaissance $\Re_{o}$, lequel représente un modèle minimal d'interprétation expliquant l'ensemble des actions observées $\mathcal{O}$ seulement s'il est borné par une borne supérieure $\nabla_{\text {sup }}$ représentant le plan partiel subsumant commun à l'ensemble des plans possibles et par une borne inférieure $\Delta_{i n f}$ représentant le schéma minimal d'intentions qui synthétise les prédictions du comportement du patient en fonction des observations. À l'aide de la relation de subsomption, cet espace $\Re_{o}$ forme une structure qui se raffinera au fur 


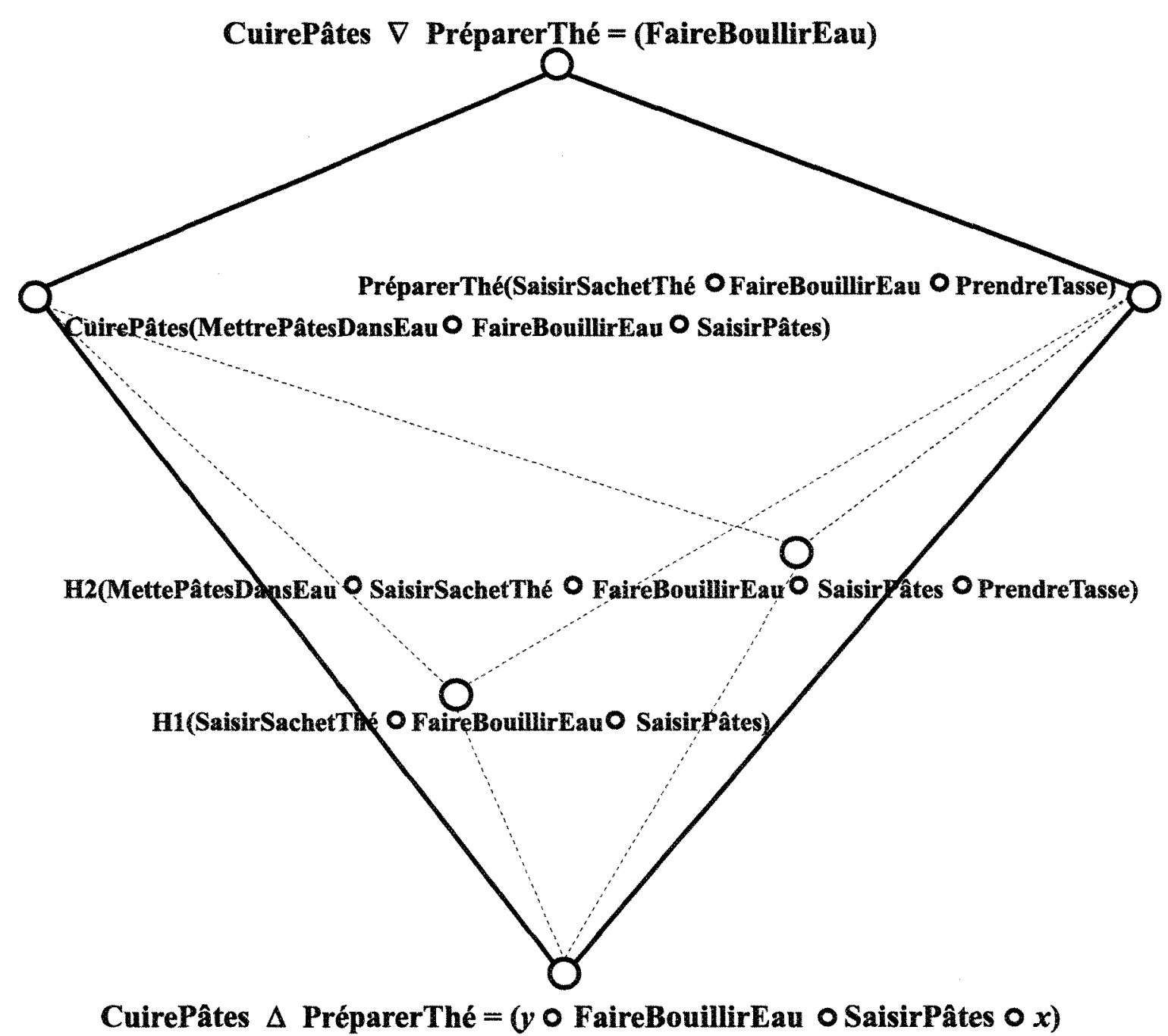

Figure 3.1 - Exemple d'un espace de reconnaissance $\Re_{o}$

et à mesure des observations. Il est composé des plans possibles en fonction des observations provenant de la librairie de plans de l'agent observateur et des hypothèses (les plans plausibles) générées par le processus de substitution, où $\Re_{o}=\left\{h \in \mathcal{P}_{l}^{\mathcal{O}} \mid \Delta_{\text {inf }} \prec_{p} h \prec_{p} \nabla_{\text {sup }}\right\}$. L'espace de reconnaissance $\Re_{o}$ constitue un outil formel permettant de caractériser et contrôler le processus de reconnaissance de plans. La Figure 3.2 résume les principales composantes du processus logique de reconnaissance de plans.

Le modèle logique présenté permet de minimiser la complexité computationnelle de l'opération de composition. En effet, un premier filtre permet de considérer les extra-plans 


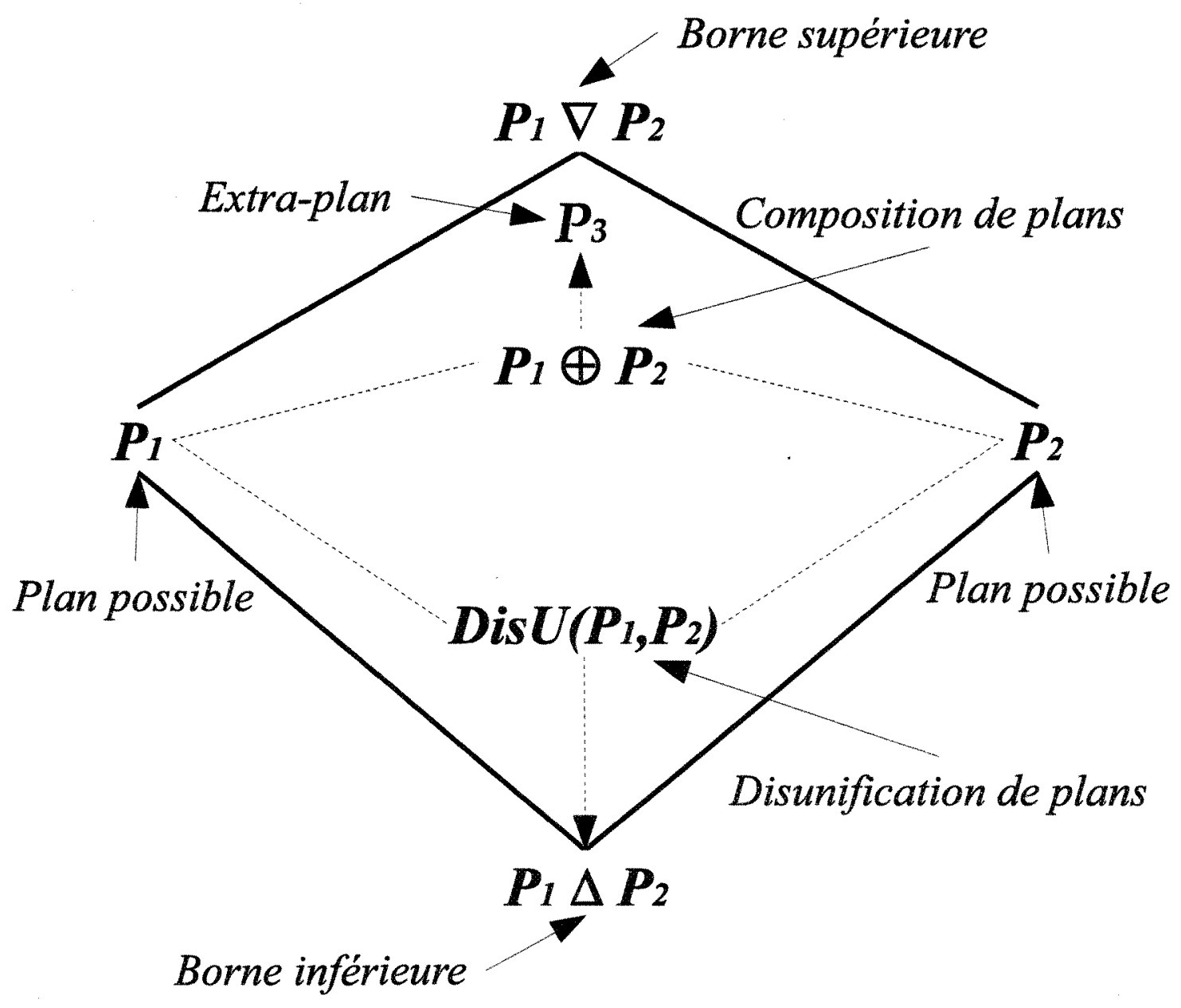

Figure 3.2 - Résumé du processus logique de reconnaissance

plausibles vérifiant les critères de stabilité et de fermeture. Un second filtre permet de prendre en compte seulement les extra-plans plausibles qui peuvent être classifiés à l'intérieur des bornes inférieure et supérieure de la structure de l'espace de reconnaissance de plans. Un troisième filtre permet de prendre uniquement en considération les extra-plans pouvant être générés par l'instanciation du schéma d'intentions à l'aide de l'opération de substitution.

Le processus logique de reconnaissance de plans permet donc de circonscrire l'ensemble des hypothèses possibles en une structure d'espace de reconnaissance. Néanmoins, toutes les hypothèses de cet espace de reconnaissance sont équiprobables, c'est-à-dire qu'il est impossible de privilégier une hypothèse par rapport à une autre. D'où l'intérêt d'une extension probabiliste 
du modèle logique qui répond à cette problématique en raffinant l'ensemble des hypothèses en fonction de leurs probabilités. Le but de la prochaine section est de présenter cette extension probabiliste du modèle logique, qui aboutira en un modèle hybride de reconnaissance de plans.

\subsection{Le modèle hybride}

L'espace de reconnaissance $\Re_{o}$ constitue une base nécessaire utilisée pour borner l'interprétation du comportement du patient. Toutefois, il ne permet pas la distinction des hypothèses pertinentes. Conséquemment, l'addition d'une quantification probabiliste sur la structure de treillis est une alternative intéressante et efficace, car elle permet de combiner l'approche symbolique pour la construction d'hypothèses avec un processus d'inférence probabiliste. La reconnaissance logique filtre les hypothèses en passant seulement un espace de reconnaissance borné au moteur d'inférence probabiliste, au lieu de considérer l'ensemble des plans de la librairie comme dans les approches probabilistes classiques.

Notre proposition consiste à introduire un ensemble de règles, inspirés des travaux sur la logique de description probabiliste [47], permettant de caractériser, à travers un intervalle de probabilités, l'influence relative d'un plan sur un autre. Elle est basée sur le degré de subsomption partielle existant entre les deux plans, même s'ils ne partagent pas, apparemment, de relation de subsomption directe.

\subsubsection{La logique de description probabiliste}

Plusieurs travaux dans le domaine de la logique de description ont pour but d'étendre cette logique pour prendre en considération différents aspects non couverts par la logique de description traditionnelle [8], comme la représentation des connaissances incertaines et vagues. Les logiques de description, dans lesquelles la sémantique est basée sur la logique du premier ordre classique, ne peuvent pas représenter les connaissances incertaines. Pour pallier à ce problème, différentes approches pour intégrer la notion probabiliste dans les logiques de description ont été proposées. On peut citer, en outre, les travaux de Heinsohn [47], de Jaeger [51], et de Koller et al. [57]. 
Les travaux de Heinsohn [47] et de Jaeger [51] utilisent les probabilités pour représenter les relations de subsomption partielle, que l'on peut dénoter par $C \stackrel{p}{\rightarrow} D$, où $C$ et $D$ sont des concepts et $p \in[0,1]$ une probabilité. Un concept $C$ est subsumé partiellement par un concept $D$ lorsqu'une fraction de l'ensemble des individus du concept $C$ est aussi dans l'ensemble des individus du concept $D$. On note que cette relation de subsomption partielle est symétrique, c'est-à-dire que si un concept $C$ subsume partiellement un concept $D$, alors un concept $D$ subsume partiellement un concept $C$. Par exemple, si un concept $D$ subsume totalement un concept $C$, c'est-à-dire que $100 \%$ des individus de $C$ sont dans $D$, d'où $C \stackrel{1}{\rightarrow} D$, alors un concept $C$ subsume partiellement un concept $D$ selon une probabilité $p$, c'est-à-dire que $(p * 100 \%)$ des individus du concept $D$ sont dans le concept $C$, d'où $D \stackrel{p}{\rightarrow} C$.

Les travaux de Jaeger [51] utilisent aussi les probabilités sur les assertions, c'est-à-dire qu'un individu $a$ fait partie d'un concept $C$ selon une probabilité $p$, dénoté par $P(C(a))=p$. Les travaux de Koller et al. [57] utilisent les réseaux bayésiens pour exprimer l'incertitude à propos des propriétés des individus.

\subsubsection{L'extension probabiliste}

L'extension probabiliste que nous proposons tente de répondre au problème d'équiprobabilité entre les hypothèses qui est cité précédemment en utilisant le degré de subsomption partielle entre deux plans, qui permet de caractériser l'influence relative d'un plan sur un autre à l'aide d'un intervalle de probabilités.

\subsubsection{Les intervalles probabilistes}

On peut principalement justifier l'utilisation d'un intervalle de probabilités plutôt qu'une probabilité ponctuelle, utilisée par des approches hybrides précédentes, par les raisons suivantes. Premièrement, on peut noter que, dans plusieurs applications, la probabilité spécifique d'un événement ne peut pas être précisément évaluée [31,33]. De plus, l'incertitude concernant les influences des extra-plans erronés versus entrecroisés, générée par le dilemme, ne peut pas être prise en compte par une probabilité ponctuelle. Selon Boole [19], lorsqu'on est dans une situation d'ignorance totale à propos de la dépendance spécifique entre deux évènements, alors 
tout ce que nous pouvons dire au sujet de la probabilité de la conjonction ou de la disjonction de ces deux évènements est qu'elle appartient à un intervalle.

\subsubsection{Explication du modèle hybride}

En utilisant le modèle logique pour générer l'espace de reconnaissance $\Re_{0}$ contenant l'ensemble des hypothèses sur le comportement du patient Alzheimer en fonction de l'ensemble des observations $\mathcal{O}$ et de la librairie de l'agent observateur (la librairie d'actions $\mathcal{A}$ et la librairie de plans $\mathcal{P}$ ), on utilise la couche probabiliste pour réduire l'ensemble des hypothèses en probabilisant la structure de l'espace de reconnaissance $\Re_{o}$. Cette section formalise ce processus hybride de reconnaissance de plans.

\section{Bases de l'extension probabiliste}

Soit une librairie de plans $\mathcal{P}=\left\{\alpha_{1}, \cdots, \alpha_{l}\right\}$ et une librairie d'actions $\mathcal{A}=\left\{a_{1}, \cdots, a_{n}\right\}$. Soit l'ensemble d'observations $\mathcal{O}=\left\{o_{1}, \cdots, o_{k}\right\}$, de sorte que $\mathcal{P}_{s}^{\mathcal{O}} \subseteq \mathcal{P}$. Pour pouvoir évaluer les différentes probabilités utilisées dans le modèle hybride, on utilise deux types de tableaux : le tableau des fréquences d'observations des plans réalisés et le tableau des fréquences de réalisations erronées et entrecroisées. On note que le patient peut réaliser une activité de façon correcte (aucune déviation du plan de l'activité) ou bien de façon erronée ou entrecroisée (déviation du plan de l'activité). Le tableau des fréquences d'observations des plans réalisés (Tableau 3.1) présente, par période d'échantillonnage ${ }^{3}$, la fréquence de réalisation des différents plans (activités) de la librairie. Pour chaque période d'échantillonnage $E_{i}$ d'une taille $T_{i}$, chacun des plans $\alpha_{j}$ possède une fréquence d'observation $F \mathrm{i}_{\alpha_{j}}$.

Le tableau des fréquences de réalisations erronées et entrecroisées (Tableau 3.2) présente, par période d'échantillonnage, la fréquence de chaque type de réalisation erronée ou de réalisation entrecroisée parmi les différents plans observés tirés du Tableau 3.2. Pour chaque période d'échantillonnage $E_{i}$ d'une taille $T_{i}$, chaque type de réalisation erronée/entrecroisée Type possède une fréquence d'observation $R \mathrm{i}_{T y p e}$.

\footnotetext{
${ }^{3}$ Une période d'échantillonnage représente, dans notre cas, un sous-ensemble des réalisations (erronées ou non) observées. Par exemple, une période d'échantillonnage peut représenter les différentes réalisations effectuées par le patient durant une semaine.
} 


\begin{tabular}{|c|c|c|c|c|c|}
\hline Plans & $\mathrm{E}_{1}$ & $\cdots$ & $\mathrm{E}_{i}$ & $\cdots$ & $\mathrm{E}_{s}$ \\
\hline \hline$\alpha_{1}$ & $F 1_{\alpha_{1}}$ & $\cdots$ & $F \mathrm{i}_{\alpha_{1}}$ & $\cdots$ & $F \mathrm{~s}_{\alpha_{1}}$ \\
\hline$\cdots$ & $\cdots$ & $\cdots$ & $\cdots$ & $\cdots$ & $\cdots$ \\
\hline$\alpha_{j}$ & $F 1_{\alpha_{j}}$ & $\cdots$ & $F \mathrm{i}_{\alpha_{j}}$ & $\cdots$ & $F \mathrm{~s}_{\alpha_{j}}$ \\
\hline$\cdots$ & $\cdots$ & $\cdots$ & $\cdots$ & $\cdots$ & $\cdots$ \\
\hline$\alpha_{l}$ & $F 1_{\alpha_{l}}$ & $\cdots$ & $F \mathrm{i}_{\alpha_{l}}$ & $\cdots$ & $F \mathrm{~s}_{\alpha_{l}}$ \\
\hline Total des observations & $T_{1}$ & $\cdots$ & $T_{i}$ & $\cdots$ & $T_{s}$ \\
\hline
\end{tabular}

Tableau 3.1 - Fréquences d'observations des plans réalisés

\begin{tabular}{|c|c|c|c|c|c|}
\hline Types de plan & $\mathrm{E}_{1}$ & $\cdots$ & $\mathrm{E}_{i}$ & $\cdots$ & $\mathrm{E}_{s}$ \\
\hline \hline Séquence & $R 1_{\text {seq }}$ & $\cdots$ & $R \mathrm{i}_{\text {seq }}$ & $\cdots$ & $R \mathrm{~s}_{\text {seq }}$ \\
\hline Réalisation & $R 1_{\text {real }}$ & $\cdots$ & $R \mathrm{i}_{\text {real }}$ & $\cdots$ & $R \mathrm{~s}_{\text {real }}$ \\
\hline Initialisation & $R 1_{\text {init }}$ & $\cdots$ & $R \mathrm{i}_{\text {init }}$ & $\cdots$ & $R \mathrm{~s}_{\text {init }}$ \\
\hline Organisation & $R 1_{\text {org }}$ & $\cdots$ & $R \mathrm{i}_{\text {org }}$ & $\cdots$ & $R \mathrm{~s}_{\text {org }}$ \\
\hline Jugement & $R 1_{\text {jug }}$ & $\cdots$ & $R \mathrm{i}_{\text {jug }}$ & $\cdots$ & $R \mathrm{~s}_{\text {jug }}$ \\
\hline Complétion & $R 1_{\text {comp }}$ & $\cdots$ & $R \mathrm{i}_{\text {comp }}$ & $\cdots$ & $R \mathrm{~s}_{\text {comp }}$ \\
\hline Entrecroisé & $R 1_{\text {ent }}$ & $\cdots$ & $R \mathrm{i}_{\text {ent }}$ & $\cdots$ & $R \mathrm{~s}_{\text {ent }}$ \\
\hline Total de l'ensemble des observations & $T_{1}$ & $\cdots$ & $T_{i}$ & $\cdots$ & $T_{s}$ \\
\hline
\end{tabular}

Tableau 3.2 - Fréquences de réalisations erronées et entrecroisées

\section{Calcul des probabilités a posteriori}

Avant de lancer le processus de reconnaissance de plans, on évalue la probabilité d'observation a posteriori des actions et des plans provenant de la librairie de l'agent observateur. Cette estimation est basée sur une base de données obtenue en entrée et composée d'échantillons de fréquences d'observations concernant la réalisation des différentes activités, lesquelles sont obtenues à la fin d'une période d'entrainement pendant que le système apprend les routines usuelles du patient. Pour chaque plan $\alpha \in \mathcal{P}$, on utilise les fréquences minimale et maximale d'observations du plan $\alpha$ provenant du Tableau 3.1 pour calculer la probabilité a posteriori $P(\alpha)$, tel que montré dans la Formule 3.5: 


$$
\begin{gathered}
F_{\min }(\alpha)=\min \left(F 1_{\alpha}, \cdots, F \mathrm{i}_{\alpha}, \cdots, F \mathrm{~s}_{\alpha}\right) \\
F_{\max }(\alpha)=\max \left(F 1_{\alpha}, \cdots, F \mathrm{i}_{\alpha}, \cdots, F \mathrm{~s}_{\alpha}\right) \\
P_{\min }(\alpha)=\frac{F_{\min }(\alpha)}{T_{\min }} \\
P_{\max }(\alpha)=\frac{F_{\max }(\alpha)}{T_{\max }} \\
P(\alpha) \in\left[P_{\min }(\alpha), P_{\max }(\alpha)\right]
\end{gathered}
$$

Pour chaque action $a \in \mathcal{A}$, on utilise les sommes minimale et maximale des fréquences d'observations des plans $\alpha_{i}$ qui contiennent l'action $a$, c'est-à-dire $a \in \alpha_{i}$, pour calculer la probabilité a posteriori $P(a)$, tel que montré dans la Formule 3.6 :

$$
\begin{gathered}
P_{\min }(a)=\min _{i=1 \grave{a} s}\left(\frac{\sum_{\left(\alpha_{j} \mid a \in \alpha_{j}\right)}\left(F \mathrm{i}_{\alpha_{j}}\right)}{\left|\mathrm{E}_{i}\right|}\right) \\
P_{\max }(a)=\max _{i=1 \grave{a} s}\left(\frac{\sum_{\left(\alpha_{j} \mid a \in \alpha_{j}\right)}\left(F \mathrm{i}_{\alpha_{j}}\right)}{\left|\mathrm{E}_{i}\right|}\right) \\
P(a) \in\left[P_{\min }(a), P_{\max }(a)\right]
\end{gathered}
$$

\section{Calcul de la probabilité d'influence d'une action}

À partir des probabilités a posteriori des actions et des plans, on calcule la probabilité d'influence qu'une action $a \in \mathcal{A}$ possède sur un plan $\alpha \in \mathcal{P}$, dénoté par $P(\alpha \mid a)$. Cette probabilité d'influence correspond à la probabilité conditionnelle d'observer la réalisation d'un plan possible $\alpha$, sachant que l'action $a$ a été observée. Pour ce faire, on se base sur le théorème de Bayes [16], tel que montré par la Formule 3.7.

$$
P(b \mid a)=\frac{P(a \mid b) * P(b)}{P(a)}
$$


En se basant sur le théorème de Bayes pour évaluer la probabilité $P(\alpha \mid a)$ en calculant $P_{\min }(\alpha \mid a)$ et $P_{\max }(\alpha \mid a)$, tel que montré par la Formule 3.8, on doit utiliser la probabilité a posteriori du plan $\alpha$, dénotée par $P(\alpha)$, la probabilité a posteriori de l'action $a$, dénotée par $P(a)$, et la probabilité conditionnelle de l'action $a$ sachant que le plan $\alpha$ est en cours de réalisation, dénotée par $P(a \mid \alpha)$. Les deux premières probabilités ont été calculées précédemment et la probabilité $P(a \mid \alpha)$ est $[1,1]$ si l'action $a$ est incluse dans les actions du plan $\alpha$ et $[0,0]$ si l'action $a$ ne fait pas partie du plan $\alpha$.

$$
\begin{gathered}
P(a \mid \alpha)= \begin{cases}{[1,1]} & \text { si } a \in \alpha \\
{[0,0]} & \text { sinon. }\end{cases} \\
P_{\min }(\alpha \mid a)=\frac{P_{\min }(a \mid \alpha) * P_{\min }(\alpha)}{P_{\min }(a)} \\
P_{\max }(\alpha \mid a)=\frac{P_{\max }(a \mid \alpha) * P_{\max }(\alpha)}{P_{\max }(a)} \\
P(\alpha \mid a) \in\left[P_{\min }(\alpha \mid a), P_{\max }(\alpha \mid a)\right]
\end{gathered}
$$

\section{Calcul de la probabilité d'influence de la borne supérieure}

En sachant la probabilité d'influence des actions sur les plans, on calcule, pour chaque paire de plans incomparables $\alpha, \beta \in \mathcal{P}_{s}^{\mathcal{O}}$, la probabilité d'influence de la borne supérieure $\alpha \nabla \beta$ sur chacun des plans $\alpha$ et $\beta$, dénotés par $P(\alpha \mid \alpha \nabla \beta)$ et $P(\beta \mid \alpha \nabla \beta)$. Deux plans $\alpha$ et $\beta$ sont incomparables lorsque chacun des plans ne subsume pas totalement l'autre plan, dénoté par $\alpha \nprec_{p} \beta$ et $\beta \nprec_{p} \alpha$. Pour calculer la probabilité d'influence de la borne supérieure $\alpha \nabla \beta$ sur un plan $\alpha$, on utilise la moyenne des $P_{\min }(\alpha \mid a)$, où $a \in \alpha \nabla \beta$, tel que montré dans la Formule 3.9 . 


$$
\begin{gathered}
P_{\min }(\alpha \mid \alpha \nabla \beta)=\frac{\sum_{a \in \alpha \nabla \beta}\left(P_{\min }(\alpha \mid \alpha)\right)}{|\alpha \nabla \beta|} \\
P_{\max }(\alpha \mid \alpha \nabla \beta)=\frac{\sum_{a \in \alpha \nabla \beta}\left(P_{\max }(\alpha \mid \alpha)\right)}{|\alpha \nabla \beta|} \\
P(\alpha \mid \alpha \nabla \beta) \in\left[P_{\min }(\alpha \mid \alpha \nabla \beta), P_{\max }(\alpha \mid \alpha \nabla \beta)\right]
\end{gathered}
$$

Pour ce qui est des probabilités $P(\alpha \nabla \beta \mid \alpha)$ et $P(\alpha \nabla \beta \mid \beta)$, on utilise la valeur de [1, 1], car les plans $\alpha$ et $\beta$ sont totalement subsumés par la borne supérieure $\alpha \nabla \beta$, de sorte que :

$$
\begin{aligned}
& P(\alpha \nabla \beta \mid \alpha) \in[1,1] \\
& P(\alpha \nabla \beta \mid \beta) \in[1,1]
\end{aligned}
$$

On peut aussi utiliser une autre notation pour les probabilités d'influence, telle que montrée dans la Formule 3.11.

$$
\begin{array}{r}
P(\alpha \mid \alpha \nabla \beta) \in\left[p_{\min 1}, p_{\max 1}\right] \equiv \alpha \nabla \beta \stackrel{\left[p_{\min 1} \cdot p_{\max 1}\right]}{\longrightarrow} \alpha \\
P(\beta \mid \alpha \nabla \beta) \in\left[p_{\min 2}, p_{\max 2}\right] \equiv \alpha \nabla \beta \stackrel{\left[p_{\min 2} \cdot p_{\max 2}\right]}{\longrightarrow} \beta \\
P(\alpha \nabla \beta \mid \alpha) \equiv \alpha \stackrel{[1,1]}{\longrightarrow} \alpha \nabla \beta \\
P(\alpha \nabla \beta \mid \beta) \equiv \beta \stackrel{[1,1]}{\longrightarrow} \alpha \nabla \beta
\end{array}
$$

\section{La probabilité d'influence de la borne inférieure}

Pour chaque paire de plans incomparables $\alpha, \beta \in \mathcal{P}_{s}^{\mathcal{O}}$, les probabilités d'influence entre les hypothèses $h$ générées par le processus logique de reconnaissance et la borne inférieure $\alpha \Delta \beta$ et entre $\alpha \Delta \beta$ et les hypothèses $h$ sont définies dans la Formule 3.12. Pour toute hypothèse $h \in \alpha \oplus \beta$, la probabilité d'influence entre la borne inférieure $\alpha \Delta \beta$ et l'hypothèse $h$, dénotée par $P(h \mid \alpha \Delta \beta)$, est $[1,1]$, car l'hypothèse $h$ subsume totalement la borne inférieure $\alpha \Delta \beta$, et la probabilité d'influence entre l'hypothèse $h$ et la borne inférieure $\alpha \Delta \beta$, dénoté par $P(\alpha \Delta \beta \mid h)$, 
est $[0,1]$, car il est certain que la probabilité d'influence se retrouve à l'intérieur de l'intervalle $[0,1]$. On peut noter que pour les hypothèses $h \notin \alpha \oplus \beta$, les probabilités d'influences $P(h \mid \alpha \Delta \beta)$ et $P(\alpha \Delta \beta \mid h)$ sont $[0,0]$.

$$
\begin{aligned}
& P(h \mid \alpha \Delta \beta) \in[1,1] \equiv \alpha \Delta \beta \stackrel{[1,1]}{\longrightarrow} h \\
& P(\alpha \Delta \beta \mid h) \in[0,1] \equiv h \stackrel{[0,1]}{\longrightarrow} \alpha \Delta \beta
\end{aligned}
$$

\section{La subsomption partielle}

La notion de subsomption partielle entre deux plans peut être formalisé par la proposition suivante :

Proposition 3.1 Soit $\alpha, \beta \in \Re_{o}$, deux hypothèses interprétant les actions observées. Le plan $\beta$ subsume partiellement le plan $\alpha$ avec un intervalle de probabilités $\left[p_{\min }, p_{\max }\right]$, qui est

dénoté par $\alpha \stackrel{\left[p_{\min }, p_{\max }\right]}{\longrightarrow} \beta$, s'il existe une borne supérieure $\alpha \nabla \beta$ de sorte que $\alpha \prec_{p} \alpha \nabla \beta$ et $\beta \prec_{p} \alpha \nabla \beta$, où :

$$
\begin{aligned}
& p_{\min }=\frac{1}{P_{\min }(\alpha \mid \alpha \nabla \beta)} * \max \left(0, P_{\min }(\alpha \mid \alpha \nabla \beta)+P_{\min }(\beta \mid \alpha \nabla \beta)-1\right) \\
& p_{\max }=\min \left(\frac{P_{\max }(\beta \mid \alpha \nabla \beta)}{P_{\min }(\alpha \mid \alpha \nabla \beta)}, 1\right)
\end{aligned}
$$

Le symbole $\rightarrow$ représente l'incertitude reliée à la relation de subsomption qui existe entre deux hypothèses (plans possibles et extra-plans). L'expression $p_{\min } \in[0,1]$ correspond à la probabilité caractérisant la borne inférieure de l'intervalle d'influence mutuelle entre les hypothèses $\alpha$ et $\beta$ qui peuvent expliquer les observations. En d'autres termes, elle modélise la probabilité minimale de réalisation d'une activité erronée/entrecroisée. Réciproquement, $p_{\max }$ représente la borne maximale de cette probabilité. Par exemple, l'expression $\alpha \stackrel{\left[p_{\min }, p_{\max }\right]}{\longrightarrow} h$ peut être interprétée comme le degré de plausibilité à l'intérieur de l'intervalle $\left[p_{\min }, p_{\max }\right]$ de 
la réalisation d'un extra-plan erroné/entrecroisé $h$, sachant que le patient avait débuté un plan possible $\alpha$, lequel peut aussi être noté $\mathrm{P}(\alpha \rightarrow h) \in\left[p_{\min }, p_{\max }\right]$. On peut noter que cette relation est symétrique, ce qui signifie que si $\alpha$ subsume partiellement $\beta$, alors $\beta$ subsume partiellement $\alpha$. Toutefois, si $\alpha$ subsume totalement $\beta$, alors $p_{\min }=p_{\max }=1$.

\section{Caicul de la probabilité de subsomption partielle entre deux plans possibles}

Pour chaque paire de plans $\alpha, \beta \in \mathcal{P}_{s}^{\mathcal{O}}$ on calcule les probabilités d'influence entre ces deux plans, dénotées par $P(\alpha \rightarrow \beta)$ et $P(\beta \rightarrow \alpha)$, en utilisant la Proposition 3.1, de sorte que la formule présentée dans la Proposition 3.1 calcule $P(\alpha \rightarrow \beta)$ et la Formule 3.13 calcule $P(\beta \rightarrow \alpha)$. On note que la Figure 3.3 présente graphiquement les éléments utilisés pour le calcul de $P(\alpha \rightarrow \beta)$. On note que pour le calcul de $P(\beta \rightarrow \alpha)$, la flèche de la probabilité $\left[p_{\min }, p_{\max }\right]$ est inversée.

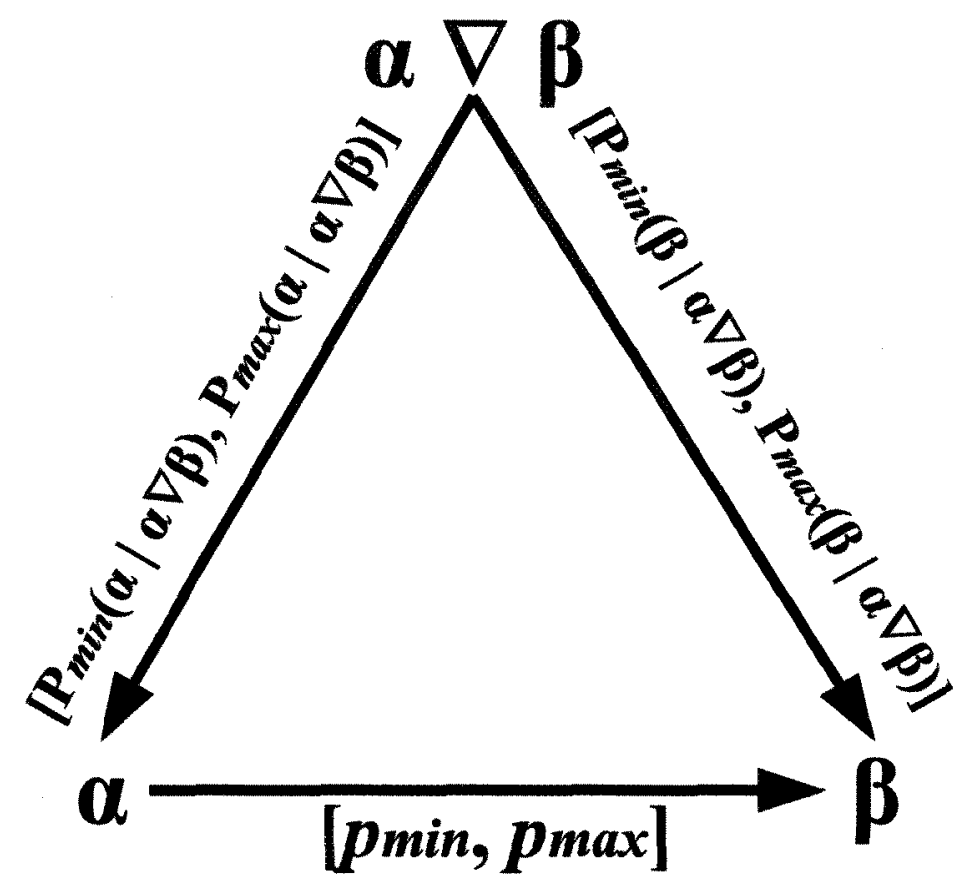

Figure 3.3 - Calcul de la probabilité $P(\alpha \rightarrow \beta)$ 


$$
\begin{gathered}
p_{\min }=\frac{1}{P_{\min }(\beta \mid \alpha \nabla \beta)} * \max \left(0, P_{\min }(\beta \mid \alpha \nabla \beta)+P_{\min }(\alpha \mid \alpha \nabla \beta)-1\right) \\
p_{\max }=\min \left(\frac{P_{\max }(\alpha \mid \alpha \nabla \beta)}{P_{\min }(\beta \mid \alpha \nabla \beta)}, 1\right) \\
P(\beta \rightarrow \alpha) \in\left[p_{\min }, p_{\text {max }}\right]
\end{gathered}
$$

Dans le cas où un extra-plan $h \in \alpha \oplus \beta$ est subsumé totalement par un seul plan possible $\alpha$ ou $\beta \in \mathcal{P}_{s}^{\mathcal{O}}$, on doit aussi calculer la probabilité $P(\alpha \rightarrow \alpha)$ et $P(\beta \rightarrow \beta)$, de sorte que :

$$
\begin{gathered}
p_{\min }=\frac{1}{P_{\min }(\alpha \mid \alpha \nabla \beta)} * \max \left(0, P_{\min }(\alpha \mid \alpha \nabla \beta)+P_{\min }(\alpha \mid \alpha \nabla \beta)-1\right) \\
p_{\max }=\min \left(\frac{P_{\max }(\alpha \mid \alpha \nabla \beta)}{P_{\min }(\alpha \mid \alpha \nabla \beta)}, 1\right) \\
P(\alpha \rightarrow \alpha) \in\left[p_{\min }, p_{\max }\right]
\end{gathered}
$$

et

$$
\begin{gathered}
p_{\min }=\frac{1}{P_{\min }(\beta \mid \alpha \nabla \beta)} * \max \left(0, P_{\min }(\beta \mid \alpha \nabla \beta)+P_{\min }(\beta \mid \alpha \nabla \beta)-1\right) \\
p_{\max }=\min \left(\frac{P_{\max }(\beta \mid \alpha \nabla \beta)}{P_{\min }(\beta \mid \alpha \nabla \beta)}, 1\right) \\
P(\beta \rightarrow \beta) \in\left[p_{\min }, p_{\max }\right]
\end{gathered}
$$

Lorsqu'on compare les probabilités $P(\alpha \rightarrow \beta)$ et $P(\beta \rightarrow \alpha)$, si $P(\alpha \rightarrow \beta)$ est plus élevée que $P(\beta \rightarrow \alpha)$, cela signifie qu'il est plus probable que le patient dévie de son intention initiale $\alpha$ vers le plan $\beta$ plutôt que le contraire. On a cette situation lorsque la probabilité a posteriori de $\beta$ est supérieure que la probabilité a posteriori de $\alpha$. En effet, lorsque le patient 
a pour intention le plan $\alpha$, son comportement aura tendance à dévier vers un plan ayant une probabilité a posteriori plus élevée, soit $\beta$. Cette déviation de l'intention initiale aura pour conséquence de produire un comportement erroné ou un comportement qui réalise de façon entrecroisée les plans $\alpha$ et $\beta$. Dans le cas contraire, si le patient a pour intention initiale le plan $\beta$, son comportement aura moins tendance à dévier vers un plan ayant une probabilité $a$ posteriori inférieure, soit $\alpha$.

\section{Calcul de la probabilité de subsomption partielle entre une hypothèse et un plan possible}

Pour chaque hypothèse $h \in \alpha \oplus \beta$, où $\alpha, \beta \in \mathcal{P}_{s}^{\mathcal{O}}$, on calcule les probabilités de subsomption partielle entre l'hypothèse $h$ et les plans possibles $\alpha$ et $\beta$, dénotées par $P(\alpha \rightarrow h)$ et $P(\beta \rightarrow h)$. Pour cela, on utilise le type de plan de l'hypothèse $h$, dont les probabilités minimale et maximale sont calculées à partir du Tableau 3.2 à l'aide de la Formule 3.16.

$$
\begin{gathered}
R_{\min }(\text { type })=\min \left(R 1_{\text {type }}, \cdots, R \mathrm{i}_{\text {type }}, \cdots, R \mathrm{~s}_{\text {type }}\right) \\
R_{\max }(\text { type })=\max \left(R 1_{\text {type }}, \cdots, R \mathrm{i}_{\text {type }}, \cdots, R \mathrm{~s}_{\text {type }}\right) \\
P_{\min }(\text { type })=\frac{R_{\min }(\mathrm{type})}{T_{\min }} \\
P_{\max }(\mathrm{type})=\frac{R_{\max }(\mathrm{type})}{T_{\max }} \\
P(\text { type }) \in\left[P_{\min }(\text { type }), P_{\max }(\text { type })\right]
\end{gathered}
$$

Les probabilités de subsomption partielle $P(\alpha \rightarrow h)$ et $P(\beta \rightarrow h)$ sont obtenues à partir de la Formule 3.17. Pour ce qui est des probabilités de subsomption entre les plans $\alpha$, $\beta$ et l'hypothèse $h$, dénotées par $P(h \rightarrow \alpha)$ et $P(h \rightarrow \beta)$, si l'hypothèse $h$ est totalement subsumée par les plans $\alpha$ et $\beta$, alors $P(h \rightarrow \alpha)=P(h \rightarrow \beta)=[1,1]$. 


$$
\begin{gathered}
P(\alpha \rightarrow \beta) \in\left[p_{\min }, p_{\max }\right] \\
P(\beta \rightarrow \alpha) \in\left[q_{\min }, q_{\max }\right] \\
P(\operatorname{type}(h)) \in\left[r_{\min }, r_{\max }\right] \\
P(\alpha \rightarrow h) \in\left[p_{\min }+\left(r_{\min } \times\left(p_{\max }-p_{\min }\right)\right), p_{\min }+\left(r_{\max } \times\left(p_{\max }-p_{\min }\right)\right)\right] \\
P(\beta \rightarrow h) \in\left[q_{\min }+\left(r_{\min } \times\left(q_{\max }-q_{\min }\right)\right), q_{\min }+\left(r_{\max } \times\left(q_{\max }-q_{\min }\right)\right)\right]
\end{gathered}
$$

\section{Interprétation des résultats}

Afin d'être en mesure d'interpréter les résultats produits par le processus de reconnaissance hybride, on doit être apte à comparer les probabilités de subsomption entre les hypothèses $h_{i} \in \mathcal{P}_{l}^{\mathcal{O}}$ et les plans possibles $\alpha_{i} \in \mathcal{P}_{s}^{\mathcal{O}}$. Pour comparer deux probabilités de subsomption, on utilise les valeurs des bornes supérieures et inférieures des intervalles de probabilité, tel que montré par la Formule 3.18

$$
\begin{gathered}
P\left(\alpha_{i} \rightarrow h_{i}\right) \in\left[p_{\min }, p_{\max }\right] \\
P\left(\alpha_{j} \rightarrow h_{j}\right) \in\left[q_{\min }, q_{\max }\right] \\
P\left(\alpha_{i} \rightarrow h_{i}\right)>P\left(\alpha_{j} \rightarrow h_{j}\right) \text { ssi } p_{\min }>q_{\min } \text { et } p_{\max }>q_{\max } \\
P\left(\alpha_{i} \rightarrow h_{i}\right) \geq P\left(\alpha_{j} \rightarrow h_{j}\right) \text { ssi } p_{\min }<q_{\max } \text { et } p_{\max }>q_{\max } \\
P\left(\alpha_{i} \rightarrow h_{i}\right)=P\left(\alpha_{j} \rightarrow h_{j}\right) \text { ssi } p_{\min }=q_{\min } \text { et } p_{\max }=q_{\max }
\end{gathered}
$$

Lorsqu'une probabilité $P\left(\alpha_{i} \rightarrow h_{i}\right)$ est supérieure $(>$ ou $\geq)$ à une autre probabilité $P\left(\alpha_{j} \rightarrow h_{j}\right)$, il faut être en mesure de l'expliquer. Plusieurs cas peuvent se présenter en fonction de l'égalité entre les plans possibles et de l'égalité entre les hypothèses:

1. $\alpha_{i} \neq \alpha_{j}$ et $h_{i}=h_{j}$ : Si le patient effectue le plan erroné (ou entrecroisé) $h_{i}$, la probabilité que son intention initiale consiste au plan $\alpha_{i}$ est plus élevée que si son intention initiale est $\alpha_{j}$. 
2. $\alpha_{i}=\alpha_{j}$ et $h_{i} \neq h_{j}$ : Quand l'intention initiale du patient consiste au plan $\alpha_{i}$, la probabilité qu'il effectue la réalisation erronée $h_{i}$ de l'intention initiale est plus élevée que s'il effectuait la réalisation erronée $h_{j}$ de l'intention initiale.

3. $\alpha_{i} \neq \alpha_{j}$ et $h_{i} \neq h_{j}$ : La probabilité que le patient ait pour intention initiale $\alpha_{i}$ et qu'il effectue la réalisation erronée (ou entrecroisée) $h_{i}$ de l'intention initiale est plus élevée que si son intention initiale est $\alpha_{j}$ et qu'il effectue la réalisation erronée (ou entrecroisée) $h_{j}$ de l'intention initiale.

\section{Suite du processus de reconnaissance de plan}

Lorsqu'on a généré l'espace de reconnaissance probabilisé pour l'ensemble des observations $\mathcal{O}_{\text {actuel }}$ durant la phase actuelle du processus de reconnaissance, dénoté par $\mathcal{L}_{\mathcal{O}_{\text {actuel }}}$, on doit l'unir avec l'espace de reconnaissance probabilisé produit lors de la phase précédente du processus de reconnaissance, dénoté par $\mathcal{L}_{\mathcal{O}_{\text {prec }}}^{\prime}$, pour obtenir l'espace de reconnaissance probabilisé final de la phase actuelle du processus de reconnaissance, dénoté par $\mathcal{L}_{\mathcal{O}_{\text {actuel }}}^{\prime}$, tel que montré par la Formule 3.19.

$$
\mathcal{L}_{\mathcal{O}_{\text {prec }}}^{\prime} \cup \mathcal{L}_{\mathcal{O}_{\text {actuel }}}=\mathcal{L}_{\mathcal{O}_{\text {actuel }}}^{\prime}
$$

Lorsqu'on effectue l'union de deux espaces de reconnaissance probabilisés, on doit recalculer la valeur des différentes probabilités pour tenir compte de l'évolution des probabilités à travers les différentes phases du processus de reconnaissance. Soit $\alpha, \beta \in \mathcal{L}_{\mathcal{O}_{\text {actuel }}}^{\prime}$, deux hypothèses dans le treillis résultant de l'union de $\mathcal{L}_{\mathcal{O}_{p r e c}}^{\prime}$ et $\mathcal{L}_{\mathcal{O}_{\text {actuel }}}$, il existe une probabilité $P^{\prime}$ dans $\mathcal{L}_{\mathcal{O}_{\text {actuel }}}^{\prime}$, dénotée par $\alpha \stackrel{P^{\prime}}{\longrightarrow} \beta$, si et seulement s'il existe une probabilité $P$, dénotée par $\alpha \stackrel{P}{\rightarrow} \beta$, dans $\mathcal{L}_{\mathcal{O}_{\text {prec }}}^{\prime}$ et/ou dans $\mathcal{L}_{\mathcal{O}_{\text {actuel }}}$. Si $P$ n'est pas présente dans l'un des treillis qui composent l'union, alors $P$ est égale à $[0,0]$ dans ce treillis. La Formule 3.20 permet de calculer la probabilité $P^{\prime}$ dans $\mathcal{L}_{\mathcal{O}_{\text {actuel }}}^{\prime}$ à partir de la probabilité $P$ dans $\mathcal{L}_{\mathcal{O}_{\text {prec }}}^{\prime}$ et $\mathcal{L}_{\mathcal{O}_{\text {actuel }}}$, pour chaque paire de plans $\alpha, \beta \in \mathcal{L}_{\mathcal{O}_{\text {actuel }}}$. 


$$
\begin{gathered}
P \in \begin{cases}{\left[p_{\min }, p_{\max }\right]} & \text { quand } P \in \mathcal{L}_{\mathcal{O}_{\text {prec }}}^{\prime} \\
{\left[q_{\text {min }}, q_{\max }\right]} & \text { quand } P \in \mathcal{L}_{\mathcal{O}_{\text {actuel }}}\end{cases} \\
P^{\prime} \in\left[\frac{\left(p_{\min } *\left|\mathcal{O}_{\text {prec }}\right|\right)+q_{\min }}{\left|\mathcal{O}_{\text {actuel }}\right|}, \frac{\left(p_{\text {max }} *\left|\mathcal{O}_{\text {prec }}\right|\right)+q_{\text {max }}}{\left|\mathcal{O}_{\text {actuel }}\right|}\right]
\end{gathered}
$$

\subsubsection{Exemple du processus hybride de reconnaissance de plans}

Afin de faciliter la compréhension du modèle hybride, cette section présente un exemple du processus hybride de reconnaissance de plans. Dans cet exemple, on généralise l'exemple présenté précédemment en supposant l'observation d'une première action FaireBouillirEau, et en présumant l'existence d'un historique d'observations des plans de la librairie de l'agent observateur. Nous avons construit un historique d'observation en s'inspirant des fréquences d'observation des différents types de comportements cohérents et erronés décrites dans l'étude de Giovannetti et al. [43], réalisée sur 51 patients réels atteints de maladies neurodégénératives (démences), lesquelles incluent la maladie d'Alzheimer. Le Tableau 3.3 permet d'indiquer quatre échantillons de fréquences de réalisation des plans de la librairie conmue de l'agent observateur, dont les plans sont : CuirePâtes, PréparerThé, et RegarderTélévision. En corrélation avec ces données, l'information donnée par le Tableau 3.4 indique la quantité des réalisations erronées/entrecroisées observées reliées à chaque échantillon.

\begin{tabular}{|c|c|c|c|c|}
\hline Plans & Éch.1 & Éch.2 & Éch.3 & Éch.4 \\
\hline \hline CuirePâtes & 55 & 50 & 47 & 50 \\
\hline PréparerThé & 30 & 35 & 40 & 35 \\
\hline RegarderTélévision & 15 & 15 & 13 & 15 \\
\hline \hline Total & 100 & 100 & 100 & 100 \\
\hline
\end{tabular}

Tableau 3.3 - Fréquences de réalisation des plans de l'exemple 


\begin{tabular}{|c|c|c|c|c|}
\hline Types de plan & Éch.1 & Éch.2 & Éch.3 & Éch.4 \\
\hline \hline Séquence & 10 & 13 & 10 & 12 \\
\hline Réalisation & 15 & 12 & 15 & 13 \\
\hline Initiation & 2 & 3 & 2 & 3 \\
\hline Organisation & 3 & 2 & 3 & 2 \\
\hline Jugement & 0 & 0 & 0 & 0 \\
\hline Complétion & 0 & 0 & 0 & 0 \\
\hline Entrecroisé & 5 & 7 & 10 & 9 \\
\hline \hline Total de l'ensemble des observations & 100 & 100 & 100 & 100 \\
\hline
\end{tabular}

Tableau 3.4 - Réalisations erronées/entrecroisées de l'exemple

À partir de cette base de connaissances, la première étape consiste à calculer la probabilité a posteriori attribuée à chacun des plans possibles inclus dans $\mathcal{P}_{s}^{\mathcal{O}}=\{$ CuirePâtes, PréparerThé $\}$, où l'ensemble des observations est $\mathcal{O}=\left\{\right.$ FaireBouillirEau $\left.\left(o_{1}\right)\right\}$. Pour accomplir cela, on extrait les fréquences minimales et maximales de chacun de plan possible pour chaque échantillon :

$$
\begin{gathered}
P_{\min }(\text { CuirePâtes })=\frac{47}{100}=0.47 \\
P_{\max }(\text { CuirePâtes })=\frac{55}{100}=0.55 \\
P_{\min }(\text { PréparerThé })=\frac{30}{100}=0.3 \\
P_{\max }(\text { PréparerThé })=\frac{40}{100}=0.4 .
\end{gathered}
$$

Ainsi, on obtient $P($ PréparerThé $) \in[0.3,0.4]$ et $P($ CuirePâtes $) \in[0.47,0.55]$. Par la suite, le processus continue en évaluant la probabilité a posteriori de la borne supérieure du treillis CuirePâtes $\nabla$ Préparer Thé, laquelle est basée sur la fréquence d'observations de l'action FaireBouillirEau :

$$
\begin{aligned}
& P_{\min }(\text { FaireBouillirEau })=\frac{55+30}{100}=0.85 \\
& P_{\max }(\text { FaireBouillirEau })=\frac{47+40}{100}=0.87
\end{aligned}
$$

de sorte que $P($ CuirePâtes $\nabla$ PréparerThé $)=P($ FaireBouillirEau $) \in[0.85,0.87]$. 
Par conséquent, les probabilités d'influence de la borne supérieure sur les deux activités sont :

$$
\begin{gathered}
P_{\min }(\text { CuirePâtes } \mid \text { CuirePâtes } \nabla \text { PréparerThé })=\frac{1 * 0.47}{0.85}=0.55 \\
P_{\max }(\text { CuirePâtes } \mid \text { CuirePâtes } \nabla \text { PréparerThé })=\frac{1 * 0.55}{0.87}=0.63 \\
P_{\min }(\text { PréparerThé } \mid \text { CuirePâtes } \nabla \text { PréparerThé })=\frac{1 * 0.3}{0.85}=0.35 \\
P_{\max }(\text { PréparerThé } \mid \text { CuirePâtes } \nabla \text { PréparerThé })=\frac{1 * 0.4}{0.87}=0.46 .
\end{gathered}
$$

de sorte que $P($ PréparerThé | CuirePâtes $\nabla$ PréparerThé $) \in[0.35,0.46]$ et $P($ CuirePâtes | CuirePâtes $\nabla$ PréparerThé) $\in[0.55,0.63]$.

Maintenant que nous avons inféré toutes les probabilités caractérisant les plans possibles, on peut alors estimer $P($ CuirePâtes $\rightarrow$ PréparerThé), laquelle constitue une mesure de l'incertitude reliée à la relation d'influence mutuelle à partir de laquelle émerge les extra-plans $H_{1}$ et $\mathrm{H}_{2}$. En se basant sur la formule donnée dans la Proposition 3.1 , on évalue $P($ CuirePâtes $\rightarrow$ PréparerThé) :

$$
\begin{aligned}
& P_{\min }(\text { CuirePâtes } \rightarrow \text { PréparerThé })=\frac{1}{0.55} * \max (0,0.55+0.85-1)=0 \\
& P_{\max }(\text { CuirePâtes } \rightarrow \text { PréparerThé })=\min \left(\frac{0.46}{0.55}\right)=0.84
\end{aligned}
$$

de sorte que $P($ CuirePâtes $\rightarrow$ Préparer Thé $) \in[0,0.84]$. Avec ce résultat, nous sommes donc capables de dériver l'intervalle de confiance caractérisant chacune des hypothèses $H_{1}, H_{2}$, ... incluses à l'intérieur du treillis de l'espace de reconnaissance $\Re_{o}$. Pour l'extra-plan $H_{1}$, si l'on suppose que l'intention inférée du patient est la structure de plan CuirePâtes, on doit alors calculer $P\left(\right.$ CuirePâtes $\left.\rightarrow H_{1}\right)$. Sachant que l'hypothèse $H_{1}$ représente une déviation entre le plan CuirePâtes et le plan PréparerThé, on doit alors utiliser le coefficient de réalisation de ce type d'erreur, spécifier dans le Tableau 3.4, afin de restreindre les bornes de l'intervalle, qui est inclus dans $P\left(\right.$ CuirePâtes $\left.\rightarrow H_{1}\right)$. Cela nous permet de caractériser l'incertitude reliée à la réalisation possible de l'extra-plan $H_{1}$, résultant de la composition des plans CuirePâtes 
et PréparerThé. Par exemple, l'hypothèse $H_{1}$ correspond à un plan erroné classifié dans la catégorie réalisation, pour laquelle le coefficient d'erreur est inclus dans $[0.12,0.15]$, de sorte que :

$$
\begin{aligned}
& P_{\text {min }}\left(\text { CuirePâtes } \rightarrow H_{1}\right)=0+(0.12 *(0.84-0))=0.1 \\
& P_{\text {max }}\left(\text { CuirePâtes } \rightarrow H_{1}\right)=0+(0.15 *(0.84-0))=0.13 .
\end{aligned}
$$

Dans ce cas, l'intervalle de probabilités $P\left(\right.$ CuirePâtes $\left.\rightarrow H_{1}\right) \in[0.1,0.13]$ est inclus dans l'intervalle $[0,0.84]$ relié à $P($ CuirePâtes $\rightarrow$ PréparerThé $)$. On peut interpréter ce résultat comme étant la prédiction d'une séquence d'actions erronée $H_{1}$, qui est un plan erroné de type réalisation, qui est inclus à l'intérieur de l'intervalle d'influence existant entre les plans possibles initiaux CuirePâtes et PréparerThé, selon l'action observée FaireBouillirEau. L'extra-plan $\mathrm{H}_{2}$ correspond à un plan entrecroisé, pour lequel son coefficient est inclus dans $[0.05,0.1]$, de sorte que :

$$
\begin{aligned}
& P_{\min }\left(\text { CuirePâtes } \rightarrow H_{2}\right)=0+(0.05 *(0.84-0))=0.04 \\
& P_{\text {max }}\left(\text { CuirePâtes } \rightarrow H_{2}\right)=0+(0.1 *(0.84-0))=0.08 .
\end{aligned}
$$

Ainsi, l'intervalle de probabilités $P\left(\right.$ CuirePâtes $\left.\rightarrow H_{2}\right) \in[0.04,0.08]$ est, dans l'ensemble, inférieur à l'intervalle $P\left(\right.$ CuirePâtes $\left.\rightarrow H_{1}\right) \in[0.1,0.13]$. Sachant maintenant que $P($ CuirePâtes $\left.\rightarrow H_{1}\right)>P\left(\right.$ CuirePâtes $\left.\rightarrow H_{2}\right)$, on peut déduire que l'hypothèse $H_{1}$ constitue une explication légèrement plus pertinente pour les observations que l'hypothèse $\mathrm{H}_{2}$. La Figure 3.4 présente un sous-ensemble (pour une meilleure visibilité) de l'espace de reconnaissance probabilisé généré par l'observation de l'action FaireBouillirEau.

\subsubsection{Bilan du processus hybride de reconnaissance de plans}

Le modèle hybride de reconnaissance de plan proposé permet de répondre à la problématique d'équiprobabilité des hypothèses de la structure d'espace de reconnaissance en ajoutant une couche probabiliste à cette structure. Les différentes relations de subsomption 


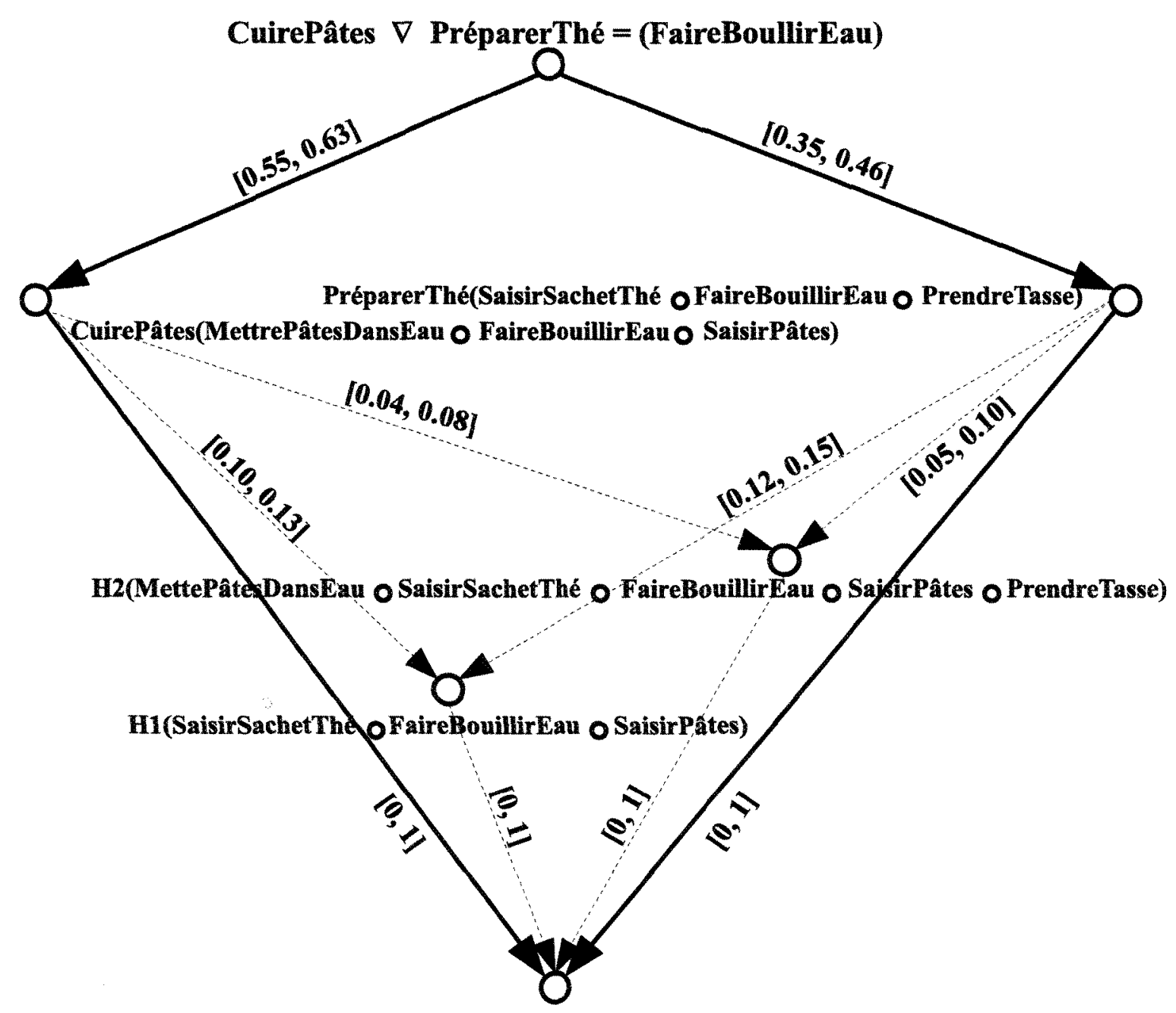

\section{CuirePâtes $\Delta$ PréparerThé $=(y \circ$ FaireBouillirEau $\circ x)$}

Figure 3.4 - Exemple d'un espace de reconnaissance probabilisé

partielles à l'intérieure de cette structure sont représentées à l'aide de probabilités, lesquelles consistent en des intervalles. Les bornes inférieure et supérieure de cet intervalle représentent les probabilités minimale et maximale qu'un plan soit subsumé par un autre plan. L'utilisation de ces probabilités permet donc de raffiner l'ensemble des hypothèses générées lors du processus logique de reconnaissance en privilégiant certaines hypothèses par rapport à d'autres.

Voici, en résumé, le déroulement du processus hybride de reconnaissance de plans. En premier lieu, la couche logique du processus de reconnaissance génère un espace de reconnaissance $\Re_{o}$, représentant les différentes hypothèses du comportement du patient en 
fonction de l'historique des observations $\mathcal{O}$ de la phase courante du processus de reconnaissance. Cette couche logique permet donc de circonscrire l'ensemble des hypothèses à l'intérieure d'une structure bornée. Dans un deuxième temps, la couche probabiliste du processus de reconnaissance prend l'espace de reconnaissance généré par la couche logique et probabilise cette structure en fonction de données d'entrée (fréquences de réalisation, ... ) pour ainsi obtenir un espace de reconnaissance probabilisé $\mathcal{L}_{o}$. Pour pouvoir tenir compte de l'évolution des probabilités à travers les différentes phases du processus de reconnaissance, on effectue l'union de l'espace de reconnaissance probabilisé $\mathcal{L}_{o}$ avec celle obtenue lors de la phase précédente du processus de reconnaissance. On obtient alors l'espace de reconnaissance probabilisé pour la phase actuelle du processus de reconnaissance, dénommé $\mathcal{L}_{o}^{\prime}$, qui tient compte des probabilités de l'ensemble des hypothèses générées par les différentes phases du processus de reconnaissance. À partir de l'espace de reconnaissance probabilisé de la phase courante $\mathcal{L}_{o}^{\prime}$, on peut sélectionner les meilleures hypothèses du comportement du patient en fonction de l'ensemble des actions observées lors du processus de reconnaissance.

\subsection{Conclusion}

Ce chapitre avait pour but de présenter un modèle hybride découlant de l'extension probabiliste d'un modèle logique basé sur un modèle d'action en logique de description et sur la théorie des treillis. L'utilisation du modèle d'action permet de transformer le problème de la reconnaissance de plan en un problème de classification. Ce modèle hybride permet de structurer le processus de reconnaissance d'activités (ou plans d'actions) d'un patient atteint de la maladie d'Alzheimer à l'intérieur d'un habitant intelligent, en identifiant un ensemble d'hypothèses pouvant expliquer le comportement du patient. La couche logique permet de circonscrire l'ensemble d'hypothèses plausibles à l'aide d'un espace de reconnaissance borné et structuré à l'aide de la relation de subsomption de plans découlant de la relation de subsomption d'actions du modèle d'action. Cependant, l'espace de reconnaissance généré par la couche logique présente une problématique d'équiprobabilité des différentes hypothèses

qui induit une incertitude concernant le comportement du patient. La couche probabiliste 
permet de réduire cette incertitude en utilisant les probabilités à l'intérieure de l'espace de reconnaissance de plans permettant de privilégier un ensemble d'hypothèses.

Lorsqu'on compare notre modèle avec les modèles hybrides de Avrahami et al. et Geib et al., on remarque que notre modèle semble a priori moins efficace du point de vue de la complexité. Ceci est causé par le fait que ceux deux approches ne génèrent pas dynamiquement des hypothèses absentes de la librairie de plans. En d'autres mots, elles suggèrent, en fonction des observations, un ensemble de plans possibles existant dans la librairie, lesquels peuvent être exécutés de manière entrecroisée. Conséquemment, ces deux approches ne prennent pas en considération le dilemme mentionné dans l'introduction, car elles considèrent que l'agent observé agit de façon rationnelle et, par conséquent, ne peut pas avoir un comportement incohérent. Donc, elles ne sont pas applicables dans un contexte d'assistance au patient Alzheimer au sein d'un habitat intelligent. Notre modèle, quant à lui, prend en considération le dilemme en générant dynamiquement des plans décrivant des comportements entrecroisés ou erronés de la part d'un patient. Ceci permet d'augmenter l'efficacité du processus de reconnaissance en augmentant sa précision dans la détection et la prédiction du comportement incohérent futur du patient. En améliorant le processus de reconnaissance, l'agent assistant est en mesure de mieux planifier ses différentes opportunités d'assistance au patient. Le prochain chapitre présentera comment ce modèle hybride de reconnaissance de plans a été concrétisé et validé à travers une application permettant la reconnaissance d'activités d'un patient atteint de la maladie d'Alzheimer à l'intérieur d'un habitat intelligent. 


\section{Chapitre 4}

\section{VALIDATION DU MODÈLE}

\subsection{Introduction}

Ce chapitre a pour but de présenter le cas de validation qui a permis de concrétiser le modèle hybride présenté au Chapitre 3. La première partie de ce chapitre permettra de présenter l'implémentation du modèle proposé. Une présentation générale du laboratoire DOMUS permettra d'illustrer comment l'implémentation du modèle hybride de reconnaissance pourrait s'arrimer au sein de la structure existante. Par la suite, l'architecture du système de reconnaissance d'activités sera présentée. Cette première partie se terminera par la présentation d'un scénario simple d'un cas de reconnaissance d'activités, permettant d'illustrer le fonctionnement de l'application.

La seconde partie du chapitre présentera la validation effectuée sur le système de reconnaissance d'activités en simulant un ensemble de scénarios tirés de l'étude de Baum et al. $[14,15]$, réalisée sur 106 patients Alzheimer, et en s'inspirant des fréquences d'observation des différents types de comportements erronés et cohérents fournies dans l'étude de Giovannetti et al. [43], réalisée sur 51 patients souffrants de maladies neurodégénératives, dont fait partie la maladie d'Alzheimer. Différents aspects de la démarche de la simulation seront présentés. En se basant sur les différents résultats de la simulation, une discussion sur les forces et faiblesses de l'approche proposée et une identification de différentes pistes de solution permettant d'améliorer le modèle seront présentées. Pour finir, une comparaison entre les résultats obtenus par notre approche et ceux obtenus par différentes approches antérieures conclura cette partie. 
L'implémentation du modèle a été effectuée en ajoutant une couche probabiliste au système de reconnaissance développé par Bruno Bouchard lors de ses travaux de thèse à 1'Université de Sherbrooke. Ce système implémente le modèle logique décrit dans les travaux de Bouchard et al. [21,22], et s'intègre à l'infrastructure du laboratoire DOMUS. La nouvelle couche du système permet d'effectuer la gestion des probabilités utilisées par le processus hybride de reconnaissance d'activités pour générer l'espace de reconnaissance contenant les différentes hypothèses sur le comportement du patient observé. L'implémentation du modèle hybride permet d'indiquer au service d'assistance les hypothèses ayant les meilleures possibilités de réalisation.

\subsection{Implémentation du modèle au sein du laboratoire DOMUS}

Le laboratoire $D O M U S^{1}$ consiste en un groupe multi-disciplinaire dédié à la recherche en domotique et en informatique mobile situé à l'Université de Sherbrooke. Les problématiques de recherche qui sont attachées à ce laboratoire sont complexes et diversifiées et recoupent plusieurs domaines (systèmes répartis, ontologies, éthique, psychologie, santé, ...). Parmi les projets en cours dans ce laboratoire, mentionnons, par exemple, l'assistance cognitive aux personnes souffrant de démence de type Alzheimer, de schizophrénie, de traumatisme crânien ou de déficience intellectuelle. Cette assistance est offerte à travers la réalisation d'un habitat intelligent et d'applications mobiles associées. Cet habitat intelligent consiste en un appartement standard avec une cuisine, un salon, une salle à manger, une chambre, et une salle de bain, qui est muni de capteurs, d'étiquettes intelligentes (RFID), de tapis sensibles à la pression, de systèmes de localisation et d'identification des personnes et objets, d'appareils audio et vidéo, etc. La Figure 4.1 illustre de façon générale l'appartement du laboratoire avec la position et la couverture des différents capteurs présents.

Dans la problématique de l'assistance à partir de l'inférence de l'activité de la vie quotidienne en cours d'exécution en fonction des actions observées, l'habitat intelligent doit alors tenir compte des aspects suivants :

\footnotetext{
${ }^{1}$ http: //domus . usherbrooke.ca
} 


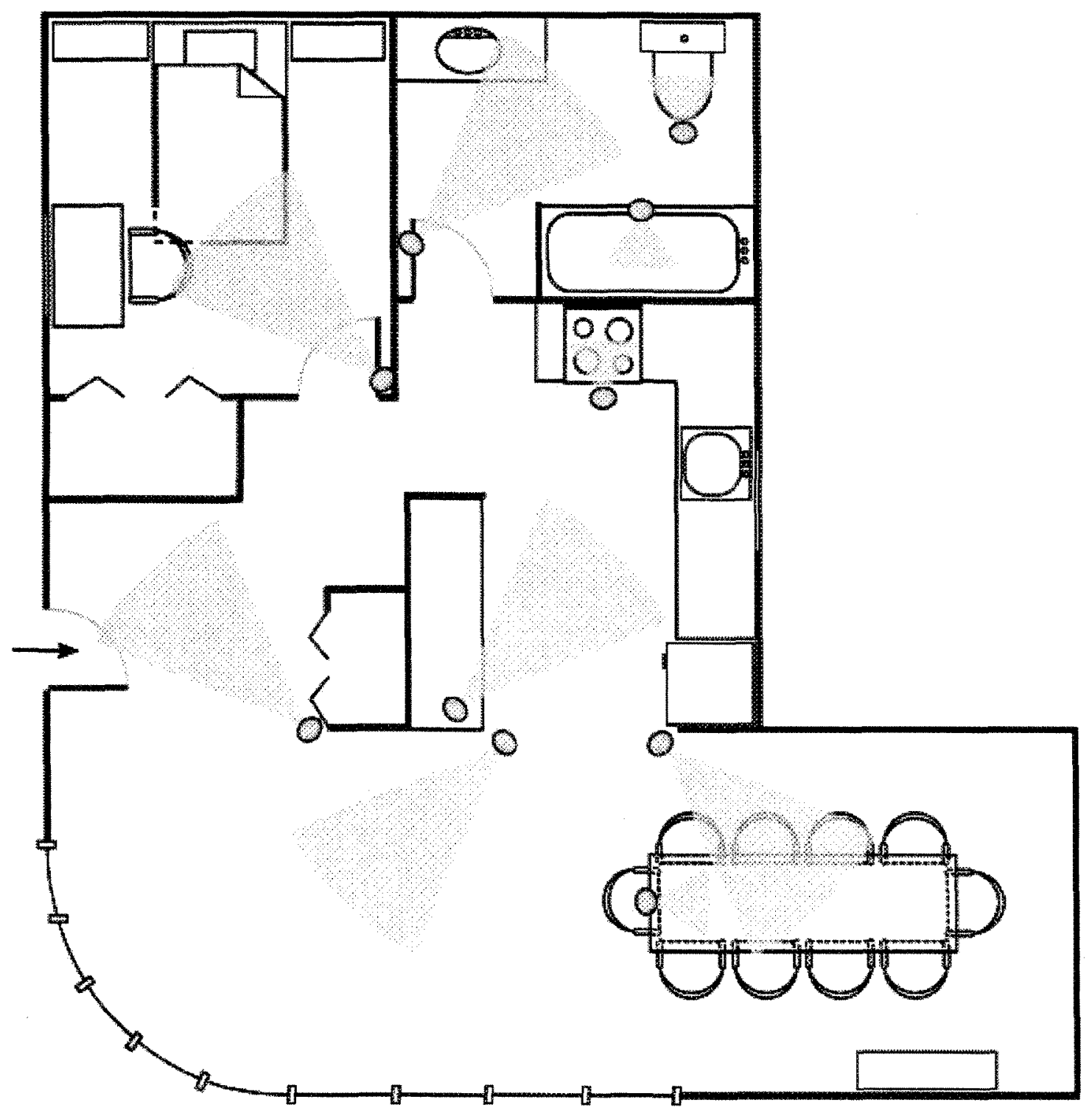

Figure 4.1 - Schéma général du laboratoire DOMUS [21] 
- Est-ce que l'occupant a besoin d'assistance? Si c'est le cas, il faut déterminer le moment opportun pour fournir de l'aide au patient.

- Il faut déterminer la manière d'assister le patient. Par exemple, l'habitat peut lui envoyer un message pour le guider dans l'exécution de l'activité en cours de réalisation, ou bien il peut effectuer directement, si c'est possible, les actions requises à la place du patient.

- Il faut déterminer quels sont les appareils (effecteurs) que l'habitat doit utiliser pour effectuer les actions d'assistances (lumières, télévision, micros, haut-parleurs, ... ). Le choix des effecteurs est réalisé en fonction des besoins du patient et de la disponibilité des différents appareils à proximité de celui-ci.

- On doit tenir compte du profil spécifique du patient qui occupe l'habitat intelligent dans le processus d'assistant. Ce profil permet à l'habitat intelligent d'établir le type particulier de déficit cognitif et les habiletés du patient.

L'implémentation du modèle hybride de reconnaissance de plans présenté dans le chapitre précédent s'intègre à l'intérieur du projet portant sur l'assistance cognitive dans le cadre d'un habitat intelligent. Cette implémentation permet, dans le cadre de la reconnaissance d'activités, de créer un modèle d'interprétation de haut niveau du comportement du patient occupant l'habitat intelligent à partir des actions observées. Ce modèle d'interprétation servira de base au système d'assistance pour lui permettre de prendre les différentes décisions se rapportant aux concepts précédemment énumérés.

\subsubsection{L'infrastructure matérielle du laboratoire}

L'infrastructure matérielle de l'appartement présent à l'intérieur du laboratoire DOMUS est basée sur une infrastructure propriétaire Crestron ${ }^{2}$. Tel que présenté par la Figure 4.2, cette infrastructure permet de contrôler et de superviser à partir d'un serveur Crestron l'ensemble des senseurs et effecteurs (système d'éclairage, dispositifs sonores, vidéo, ...) présents dans l'appartement.

\footnotetext{
${ }^{2}$ http://www.crestron.com/
} 


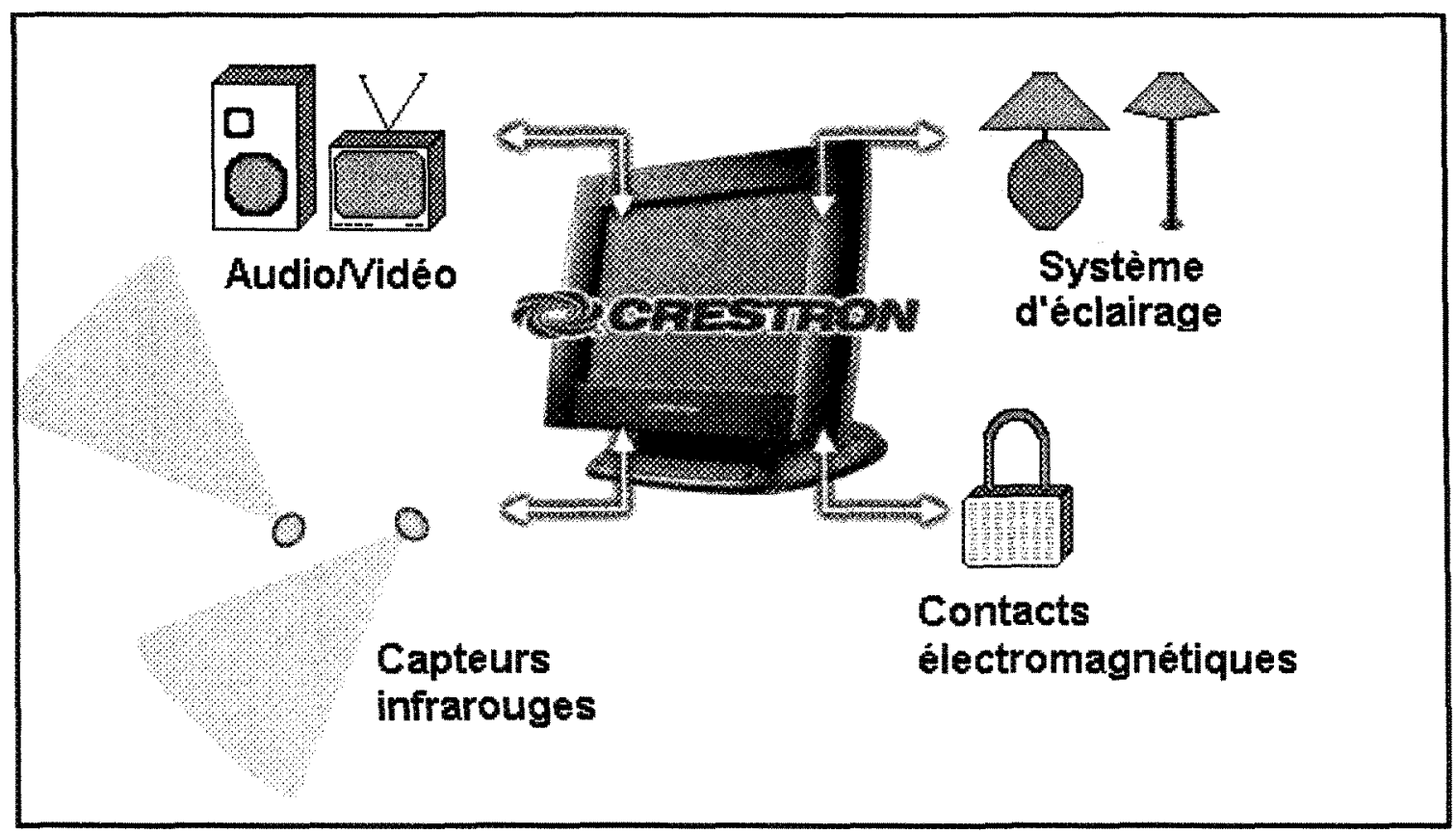

Figure 4.2 - Schéma de l'architecture Crestron [21]

Les senseurs répartis à l'intérieur de l'habitat consistent en :

- des détecteurs de mouvements,

- des contacts électromagnétiques (portes d'armoire, d'entrée, du réfrigérateur, ...),

- un système d'éclairage,

- des tapis tactiles sensibles à la pression,

- et des étiquettes intelligentes de type RFID.

Les effecteurs répartis à l'intérieur de l'habitat intelligent consistent en :

- des SmartBoards,

- des TabletPC,

- $\operatorname{des} P D A$,

- des signaux lumineux émis par le système d'éclairage,

- et des indications sonores envoyées par les haut-parleurs disposés dans chaque pièce. 


\subsubsection{Architecture de la couche logicielle de bas niveau}

L'infrastructure matérielle de l'appartement sert de point d'entrée au processus de reconnaissance d'activités. En effet, tel que présenté par la Figure 4.3, l'appartement représente l'environnement avec lequel l'occupant interagit lors de l'exécution d'une activité. Durant le déroulement d'une activité, le patient active une série de senseurs (capteurs infrarouges, tapis tactiles, étiquettes $R F I D, \ldots$ ), lesquels génèrent des évènements de bas niveau qui peuvent être envoyés directement à tous les agents connectés au serveur Crestron. Un agent de reconnaissance de bas niveau ( L Low-level Activity Regognition agent " ou $L A R$ ) reçoit les évènements produits pour ensuite effectuer la correspondance entre ces évènements de bas niveau et les actions de base que le patient peut effectuer. Ces actions font partie de la base de connaissances de l'habitat intelligent (la librairie d'actions).

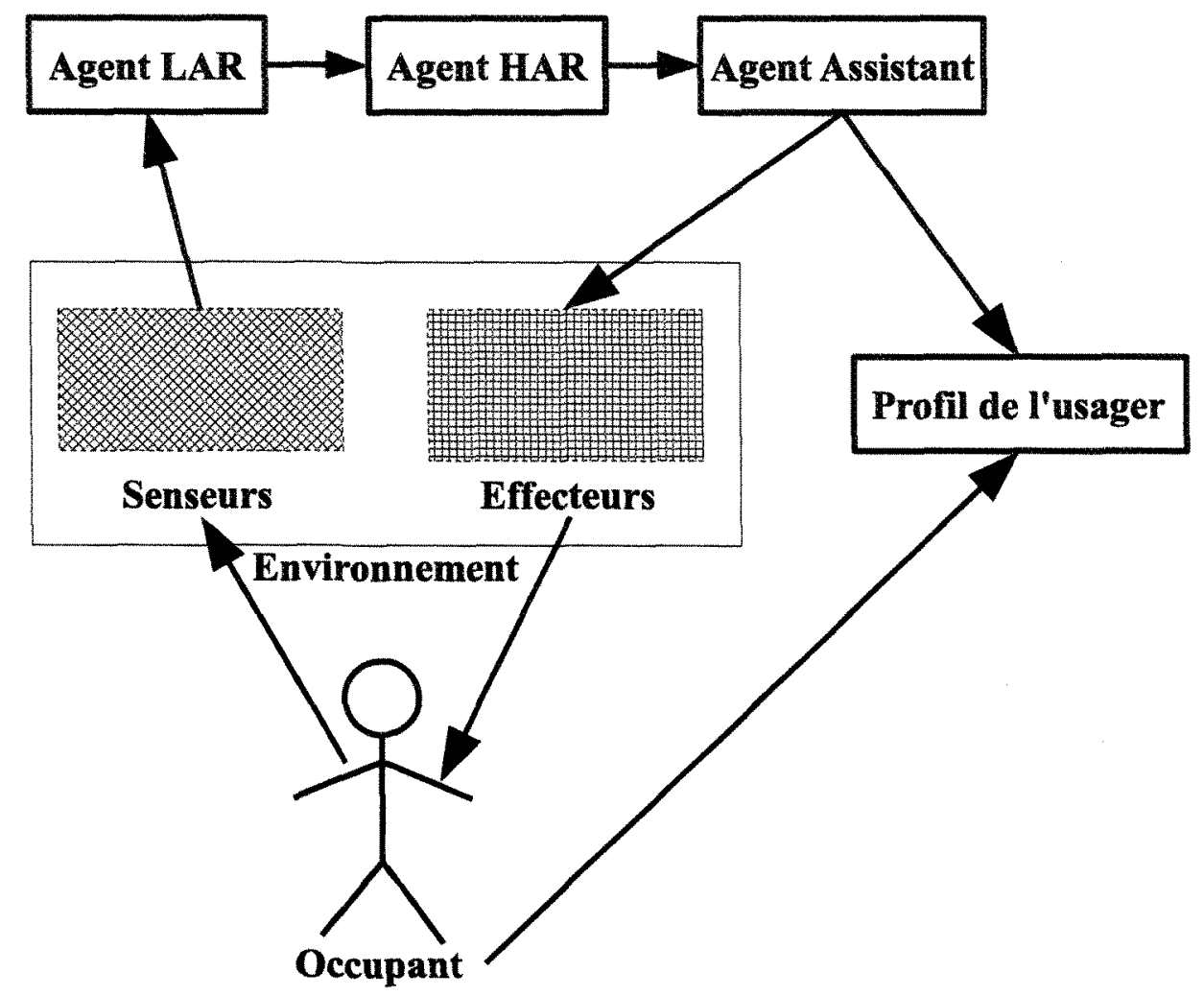

Figure 4.3 - Architecture de la couche de bas niveau 
L'agent $L A R$ utilise une représentation virtuelle de l'environnement (l'appartement) pour déterminer l'action observée en fonction des changements de l'état de l'environnement. Cette représentation virtuelle est décrite à l'intérieur du système de représentation de connaissances PowerLoom ${ }^{3}$ [26]. Ce système fournit un langage et un environnement pour construire des applications basées sur la représentation de connaissances en logique de description $[59,60]$. Par conséquent, la base de connaissances de l'agent $L A R$ est constituée d'un ensemble d'objets conceptuels et assertionnels, décrit directement dans PowerLoom, permettant de décrire l'état courant de l'environnement. Lorsque des évènements de bas niveau sont détectés à partir des senseurs, l'agent $L A R$ effectue une mise à jour de l'état de l'appartement virtuel et crée une structure d'action représentant les changements de l'état courant par rapport à l'état précédent en suivant le modèle décrit au Chapitre 3 . La structure d'action générée est ensuite classifiée à travers la taxonomie d'actions connues de l'agent $L A R$ afin de déterminer le type d'action. Après avoir identifié le type d'action, l'agent $L A R$ indique aux agents de plus haut niveau la détection d'une nouvelle action de bas niveau et envoie le type de l'action. Dans notre cas, l'agent de reconnaissance de haut niveau ( High-level Activity Regognition agent » ou HAR) et l'agent assistant sont les seuls agents de haut niveau présents. L'agent $H A R$ a pour but de déterminer un ensemble d'hypothèses sur le comportement du patient observé. À partir des hypothèses générées par l'agent de reconnaissance de haut niveau et du profil du patient, l'agent assistant doit déterminer et planifier les différentes opportunités d'assistance au patient. Ce projet de recherche ne portant pas sur l'aspect de la fiabilité du matériel, on suppose que les données envoyées par les capteurs sont correctes et complètes. Cet aspect est traité par d'autres projets de recherche au laboratoire DOMUS afin de minimiser l'incertitude reliée à la couche de bas niveau (pannes, défaillances, ...).

\subsubsection{Architecture du système de reconnaissance d'activités}

Lorsque l'agent $L A R$ détermine l'action observée de bas niveau, il envoie cet action à l'agent de reconnaissance de haut niveau. L'agent $H A R$ utilise le processus de reconnaissance

\footnotetext{
${ }^{3}$ http://www.isi.edu/isd/LOOM/PowerLoom/
} 
d'activités pour générer en ensemble d'hypothèses sur le comportement du patient en se basant sur l'ensemble des observations. Par après, l'agent $H A R$ envoie le résultat du processus de reconnaissance d'activités à l'agent assistant, lequel doit déterminer si le patient a besoin d'assistance à partir des hypothèses sur le comportement du patient. Ainsi, le processus de reconnaissance des actions de bas niveau et des activités est transparent pour l'agent assistant. L'agent HAR est implémenté à partir du modèle hybride de reconnaissance de plans présenté au Chapitre 3. Tel que l'on peut le voir à l'intérieur de la Figure 4.4, le processus de reconnaissance d'activités de l'agent HAR a été éclaté, afin d'illustrer comment celui-ci s'intègre à l'intérieur de la tâche d'assistance de l'agent assistant. L'agent $H A R$ possède une librairie de plans qui décrit les différentes activités que le patient peut effectuer à l'intérieur de l'appartement. Ces activités sont représentées à l'aide du modèle de structure de plans basé sur la logique de description.

Dans cette architecture de système d'assistance, le processus de reconnaissance des activités de la vie quotidienne s'effectue de la manière suivante. En utilisant la relation de subsomption d'actions, l'agent $H A R$ détermine quels sont les plans (activités) de la librairie qui peuvent expliquer l'ensemble des actions observées. Ces plans possèdent à l'intérieur de leur séquence au moins l'une des actions observées, et constituent ainsi l'ensemble de plans possibles. À partir de cet ensemble de plans possible, le système de reconnaissance organise les plans possibles en une structure de treillis en utilisant l'opérateur de la borne inférieure $\Delta$ et l'opérateur de la borne supérieure $\nabla$ entre chaque paire de plans. La borne inférieure entre deux plans a priori incomparables est obtenue à l'aide de l'opérateur de disunification $D i s U$, lequel permet de d'identifier les relations entre ces deux plans. Cette structure de treillis forme le cadre de l'espace de reconnaissance qui permet de borner les hypothèses générées dans la prochaine étape. En utilisant cette structure d'espace de reconnaissance, le système utilise le schéma d'intentions (la borne inférieure du treillis) pour réunifier les plans possibles à travers l'opération de composition $\oplus$. Cette opération permet, eñ utilisant le processus de substitution des variables d'actions présentes à l'intérieur du schéma d'intentions, de générer un ensemble d'extra-plans correspondant aux possibles déviations ou entrecroisements futurs du 


\section{Agents de haut niveau}

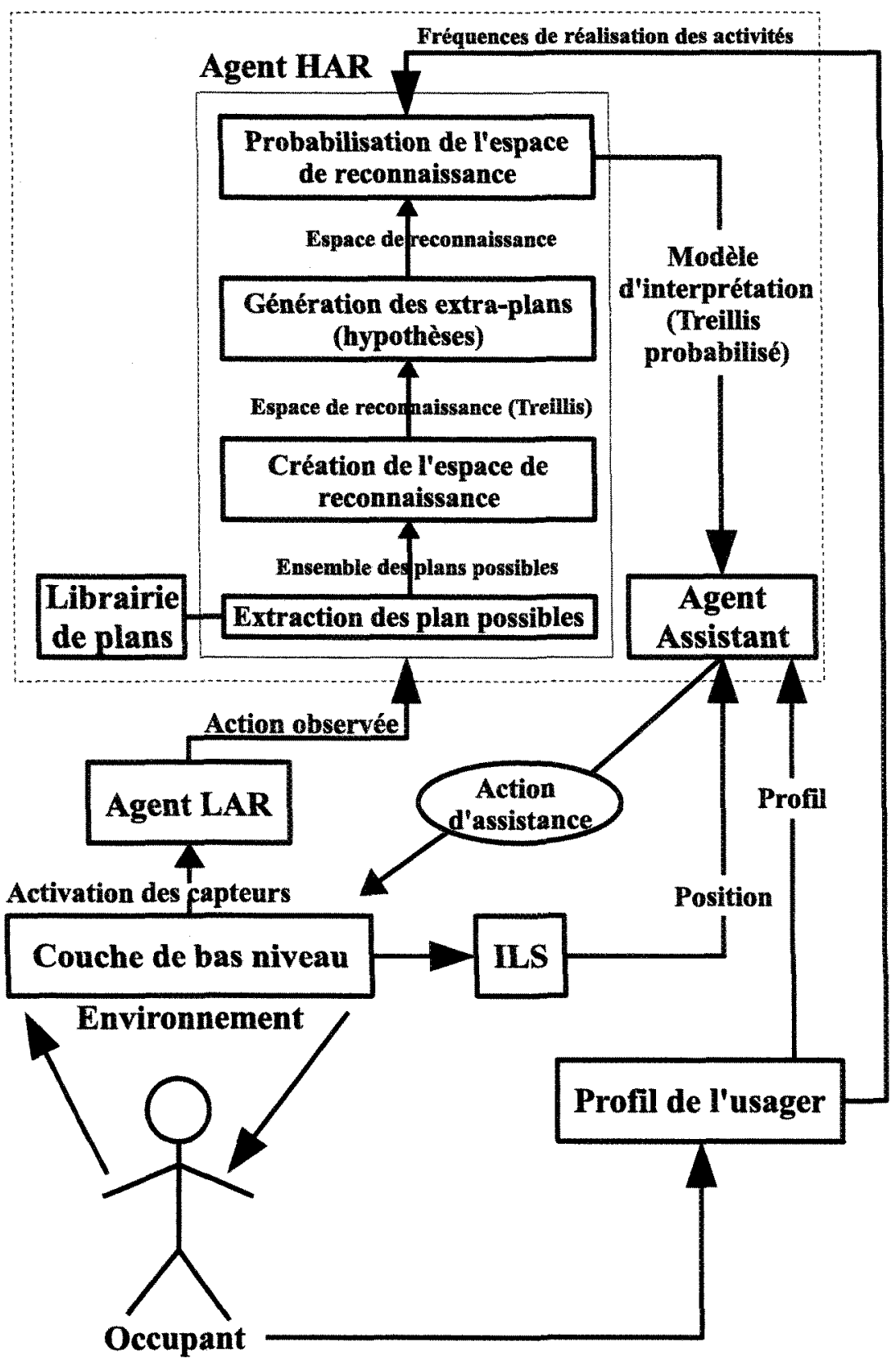

Figure 4.4 - Architecture de l'agent de reconnaissance de haut niveau 
patient. Ces extra-plans sont classifiés à l'intérieur de la structure d'espace de reconnaissance en utilisant la relation de subsomption de plans. Cette structure d'espace de reconnaissance représente donc les différentes hypothèses sur le comportement du patient lorsqu'il réalise des activités de la vie quotidienne. Afin de permettre à un sous-ensemble d'hypothèses d'être favorisé, le système de reconnaissance probabilise l'espace de reconnaissance en utilisant les fréquences de réalisation des différentes activités obtenues à partir du profil du patient.

Finalement, le modèle d'interprétation du comportement du patient (l'espace de reconnaissance probabilisé) est envoyé à l'agent assistant. Présentement, on ne s'occupe pas du volet assistance dans l'architecture du système, mais on fournit un support pour permettre à l'agent d'assistance de prendre les bonnes décisions au moment opportun grâce au modèle d'interprétation généré par l'agent $H A R$. L'agent assistant utilisera, conjointement à ce modèle d'interprétation, les informations obtenues à partir du service de localisation des objets (ILS) et du profil du patient, dans le but de déterminer les différentes opportunités d'assistance ainsi que les divers moyens à sa disposition pour effectuer ces tâches d'assistances. Dans le cas où l'agent devra assister le patient, il pourra planifier cette action d'assistance et prévoir son intervention.

\subsubsection{Outil virtuel pour la réalisation des tests}

L'utilisation d'un outil de simulations graphiques au sein du laboratoire DOMUS permet de rapidement tester certaines composantes du système de l'habitat intelligent et d'éviter certaines problématiques. En effet, lors des expérimentations du système, on ne peut pas toujours avoir recourt à des personnes atteintes de déficience cognitive ou à des membres de l'équipe du DOMUS pour réaliser ou simuler des activités de la vie quotidienne à l'intérieur de l'appartement. Ces expérimentations dans un environnement réel posent plusieurs problématiques : fatigue et disponibilité des patients, problèmes reliés à l'éthique, présence d'une ressource humaine supplémentaire, impossibilité d'effectuer des tests simultanés, etc.

Cet outil de simulation graphique, illustré dans le coin supérieur droit de la Figure 4.5, a été développé en $S V G$ par Jérémy Bauchet lors d'un projet portant sur la modélisation des 
activités de la vie quotidienne [12,13]. Le langage standard "Scalable Vector Graphics », ou $S V G^{4}$, permet de décrire des objets graphiques à deux dimensions en $X M L$. L'outil développé permet de simuler l'activation des différents capteurs présents dans l'appartement en cliquant sur la représentation graphique des différents objets de l'appartement ayant un senseur. Ensuite, le serveur de l'habitat intelligent reçoit cette "simulation " de l'activation des capteurs et, tout comme dans le cas d'une expérimentation dans un contexte réel, génère un évènement de bas niveau qui sera envoyé à l'agent $L A R$. Cet outil permet de simuler le déroulement de différents scénarios de cas réels à l'intérieur de l'habitat.

\subsubsection{Exemple de reconnaissance d'une activité de la vie quotidienne}

Dans le but d'illustrer le fonctionnement du processus de reconnaissance de plans hybride utilisé par l'agent assistant, le scénario suivant, inspiré des exemples présentés au Chapitre 3 , détaille, étape par étape, le déroulement du processus d'inférence de notre application. En début d'après-midi, Pierre se réveille au terme d'une bonne sieste, se lève de son lit et se dirige vers la cuisine. À cet instant, le tapis tactile placé à la base du lit permet à l'agent de détecter le réveil de Pierre et d'activer le système de reconnaissance d'activités. Le capteur de mouvement situé à l'entrée de la cuisine indique que Pierre vient de pénétrer dans cette pièce. Peu de temps après, le contact électromagnétique placé sur l'armoire où Pierre range les tasses montre que la porte de cette même armoire vient d'être ouverte. Ensuite, le système de localisation des objets montre qu'une tasse est actuellement en mouvement et a donc été saisie par Pierre.

À ce moment, l'agent de reconnaissance d'actions de bas niveau (agent $L A R$ ) perçoit l'activation des capteurs à travers l'infrastructure Crestron, et conceptualise alors les changements qui viennent juste de se produire à l'état de l'environnement en une structure d'action. Il classifie alors cette structure à travers sa taxonomie de manière à identifier le type de l'action observée, soit dans le cas présent PrendreTasse. Cette conceptualisation de l'action qui a été exécutée par Pierre est obtenue en classifiant de manière traditionnelle, grâce à la relation de

\footnotetext{
${ }^{4}$ http://www.w3.org/Graphics/SVG/
} 
subsomption de la logique de description, les concepts décrivant les objets impliqués dans les évènements de bas niveau générés par les divers senseurs et autres instruments d'identification et de localisation des objets et de l'équipement présent dans l'appartement du patient. À partir de cette observation, l'agent HAR déduit, à partir de sa librairie de plans, que l'ensemble des plans possibles est constitué uniquement de l'activité PréparerThé. Par conséquent, l'espace de reconnaissance pour cette observation sera composé que d'une seule hypothèse sur le comportement de Pierre : il a l'intention de se préparer un thé.

Reprenons maintenant la suite de l'exemple de Pierre, et supposons que l'agent $L A R$ détermine que la deuxième observation est l'action FaireBouillirEau. En utilisant sa base de connaissance, l'agent $H A R$ déduit alors que ces observations peuvent être réalisées pour plusieurs fins, par exemple, préparer une tasse de thé (PréparerThé) ou cuire un plat de pâtes (CuirePâtes). Pour planifier une future tâche d'assistance, l'agent doit comprendre les intentions de Pierre en construisant un modèle minimal d'interprétation (espace de reconnaissance) décrivant l'ensemble des hypothèses pouvant expliquer son comportement à partir des actions observées jusqu'à présent. Donc, à partir de l'ensemble des plans possibles, l'agent de reconnaissance construit l'espace de reconnaissance en comparant chaque paire de plans possibles (ici PréparerThé et CuirePâtes) et génère l'ensemble des extra-plans pouvant être classifiés à l'intérieur de l'espace de reconnaissance. Pour permettre à l'agent assistant de privilégier certaines hypothèses pour planifier sa future tâche d'assistance, les fréquences de réalisation des différentes activités sont utilisées pour probabiliser les éléments de l'espace de reconnaissance. Cet espace de reconnaissance probabilisé pourra être utilisé par l'agent d'assistance pour déterminer les différentes opportunités d'assistance.

La Figure 4.5 présente une copie d'écran de notre application de reconnaissance hybride lorsqu'on simule ce scénario. Sur la partie gauche de cette figure, on peut voir la description des actions de bas niveau ayant été reconnues par le système. Sur la partie inférieure droite de l'image, on peut apercevoir le treillis décrivant l'espace de reconnaissance résultant du processus de reconnaissance des plans de haut niveau. Ce treillis décrit à l'intérieur de l'application par un fichier en format GML ("Graph Modeling Language») [48]. Sur la partie supérieure droite 
de la figure, on observe l'outil graphique en $S V G$ (montrant ici le schéma de la cuisine) qui permet de simuler l'activation des différents capteurs présents dans l'habitat en cliquant sur la représentation d'un objet sur lequel est placé un capteur.

Dans la Figure 4.5, on observe que l'espace de reconnaissance (treillis) $\Re_{o}$ généré par le processus de reconnaissance hybride contient deux extra-plans : $H_{1}$ et $H_{2}$. Pour des raisons de lisibilité, on ne considère que ces deux extra-plans parmi l'ensemble des extra-plans (22) générés dynamiquement inclus dans l'espace de reconnaissance $\Re_{0}$. La première hypothèse $H_{1}$ consiste à une erreur de réalisation de la part du patient dans laquelle il effectue l'activité PréparerThé et dévie vers l'activité CuirePâtes. La deuxième hypothèse $\mathrm{H}_{2}$ consiste à une réalisation entrecroisée des activités PréparerThé et CuirePâtes.

Maintenant, supposons que Pierre effectue une troisième action, dénommée MettrePâtesDansEau, qui est complètement en dehors de la portée de l'intention initialement inférée PréparerThé. L'action MettrePâtesDansEau est, en réalité, une étape du plan CuirePâtes. Dans ce cas, la séquence d'actions observées ne correspond plus à aucun plans possibles inclus dans la librairie de l'agent observateur. Ainsi, on fait face ici au dilemme cité en introduction, qui apparaît entre les plans plausibles erronés et entrecroisés. Est-ce que cette nouvelle action dans la séquence constitue une réalisation erronée, telle que décrite par l'extra-plan $H_{1}$, ou bien est-ce que cette action constitue le commencement d'un second plan cohérent réalisé de façon entrecroisée avec le premier plan, telle que décrite par l'extra-plan $\mathrm{H}_{2}$ ?

Si l'intervalle probabiliste relié à l'hypothèse $H_{1}$, lequel correspond à $P($ Préparer Thé $\rightarrow H_{1}$ ), est dans l'ensemble supérieur à celui relié à l'hypothèse $H_{2}$, lequel correspond à $P\left(\right.$ PréparerThé $\rightarrow H_{2}$ ), alors l'hypothèse $H_{1}$ sera considérée plus pertinente. Dans cette situation, on considère que l'exécution de l'action MettrePâtesDansEau est probablement une erreur de réalisation de la part du patient. Conséquemment, l'agent d'assistance peut être autorisé à prendre le contrôle de l'habitat dans le but de planifier une action d'assistance au patient.

Par exemple, l'une de ces actions d'assistance que l'agent doit privilégier est d'indiquer au patient de fermer la cuisinière. Il est préférable que l'agent suggère une tâche plutôt que 


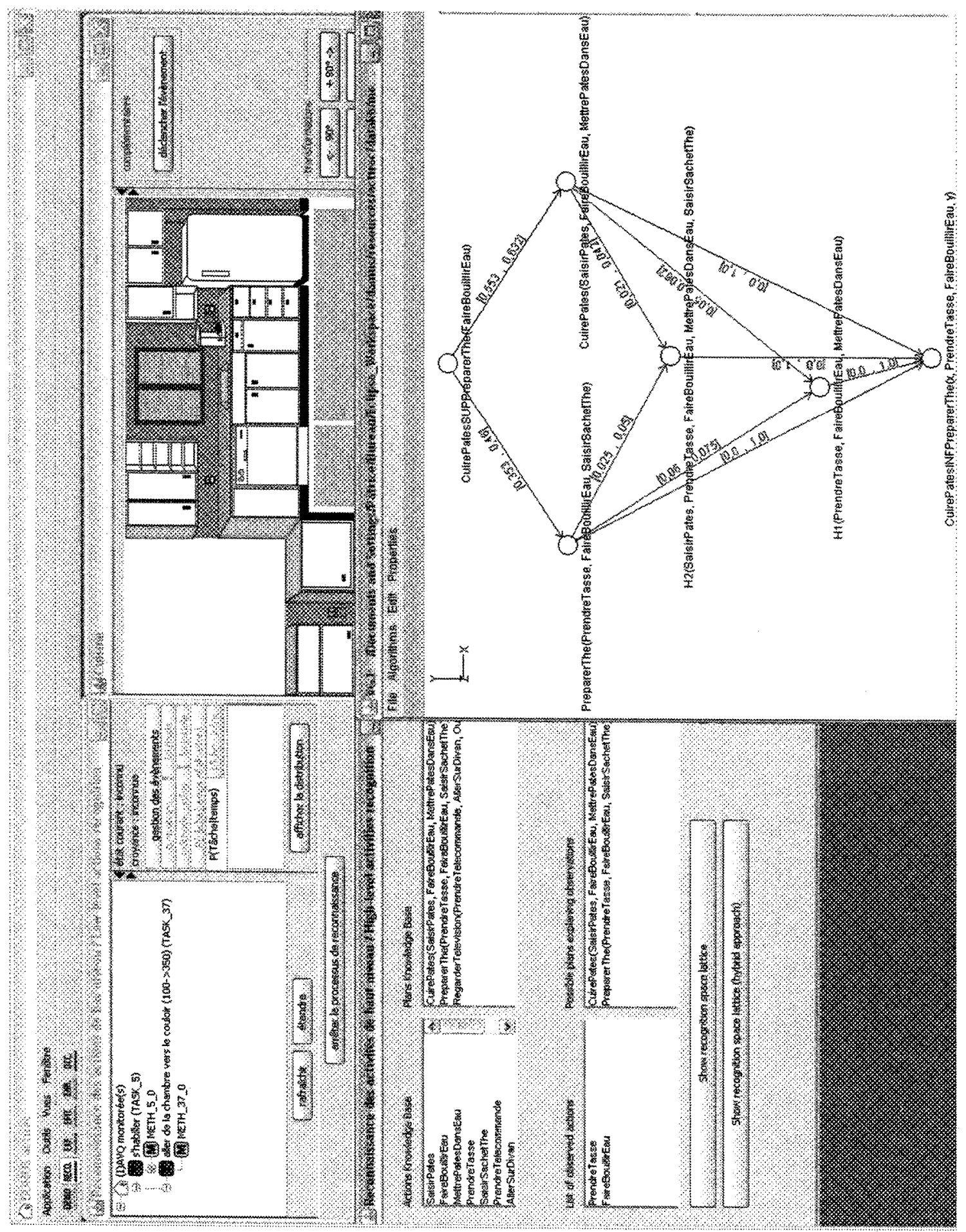

Figure 4.5 - Un exemple de reconnaissance d'une tâche de cuisine 
de l'effectuer, parce que le fait d'effectuer la tâche à la place du patient atteint de la maladie d'Alzheimer peut contribuer à la dégénérescence de sa maladie [72]. Par ailleurs, considérant qu'il existe plusieurs options d'assistance pour une même situation, un modèle basé sur la classification permet d'être utile, en plus de la reconnaissance des activités de la vie quotidienne de l'usager à travers la construction d'un modèle d'interprétation minimale, pour l'identification des différentes actions d'assistance possibles pour un même cas.

\subsection{Vérification du modèle par simulation de scénarios}

Le principal objectif de la phase de vérification du mỏèle est d'évaluer les capacités de l'agent observateur, lequel utilise le modèle de reconnaissance hybride, de compléter dynamiquement sa librairie d'activités connues de manière à prévoir les comportements incohérents ou non du patient. Chaque comportement évalué (scénario) consiste en une séquence d'actions qui décrit une réalisation erronée ou entrecroisée d'une activité. Parce que le résultat du processus de reconnaissance consiste en un ensemble d'hypothèses plausibles sur le comportement du patient, il faut donc vérifier si le modèle hybride a été en mesure de générer le plan plausible correspondant au scénario en cours d'exécution avant la fin de celui-ci. Le second objectif de la vérification consiste à évaluer empiriquement les performances au niveau du temps de calcul du modèle hybride, ainsi que la proportion d'hypothèses que l'on doit privilégier par rapport à l'ensemble des hypothèses générées par le processus de reconnaissance.

Notre simulation est basée sur l'étude de Baum et al. [14,15] effectuée sur 106 patients, âgés entre 53 ans et 86 ans, souffrants de la maladie d'Alzheimer. Dans cette étude, la performance cognitive de chaque patient Alzheimer a été évaluée en utilisant l'évaluation d'une tâche effectuée au sein d'une cuisine ( Kitchen Task Assessment » ou KTA). Le test KTA est une mesure fonctionnelle bien connue en ergothérapie, utilisée par les intervenants pour évaluer les capacités cognitives d'une personne atteinte du syndrome de Démence Sénile de Type Alzheimer (DSTA), lors de la réalisation d'une activité impliquant la préparation d'une recette de cuisine. L'objectif de ce test est de permettre au thérapeute d'identifier le niveau 
d'assistance requis par le patient afin d'être en mesure de guider les aidants naturels et les professionnels de la santé.

Cette évaluation a permis d'identifier 17 types d'erreurs communs par les patients, lesquels sont regroupés dans les six catégories d'erreurs présentées au Chapitre 1 : initiation, organisation, réalisation, séquence, jugement, et complétion. À partir de ce point, on a modélisé chacune de ces erreurs communes dans un scénario distinct. En addition de ces scénarios erronés, on a modélisé trois scénarios entrecroisés cohérents, chacun basé sur la réalisation simultanée de deux activités présentes dans la librairie de l'agent observateur. Ensuite, on a simulé l'exécution de chaque scénario, erroné et entrecroisé, étape par étape. Pour cette simulation, on a utilisé une base de connaissances constituée de quarante actions de bas niveau (librairie d'actions) et de dix activités de la vie quotidienne (librairie de plans). Chacune de ces activités correspond à une tâche normalement effectuée au sein d'une cuisine. Ces activités peuvent partager plusieurs actions entre elles, afin de créer un contexte réaliste où les plans peuvent s'entrecroiser et peuvent mener à plusieurs types d'erreurs de planification différents. Voici les différentes activités incluses à l'intérieure de la librairie de plans :

1. confectionner un gâteau,

2. cuire des pâtes,

3. préparer une tasse de thé,

4. préparer un verre de lait,

5. confectionner des crêpes,

6. cuire une omelette,

7. cuire des biscuits,

8. confectionner du sucre à la crème,

9. faire chauffer de la soupe,

10. et cuire de la viande.

Pour les différents scénarios modélisés, on considère que l'intention initiale du patient 
est de confectionner un gâteau. Voici la liste des vingt scénarios utilisés lors de la vérification par simulation du modèle hybride de reconnaissance de plans :

\section{- Initiation :}

- Scénario 1 : Le patient ne commence pas la tâche.

\section{- Organisation :}

- Scénario 2 : Le patient s'arrête au milieu de la première action.

- Scénario 3 : Le patient utilise un mauvais récipient pour mettre la recette au four.

- Scénario 4 : Le patient utilise un mauvais contenant pour mesurer du lait.

\section{- Réalisation :}

- Scénario 5 : Le patient oublie de mesurer le lait.

- Scénario 6 : Le patient oublie de mélanger les ingrédients.

- Scénario 7 : Le patient oublie de faire cuire la recette lorsqu'elle est dans le four.

- Scénario 8 : Le patient oublie de mettre dans le four la recette.

\section{- Séquence :}

- Scénario 9 : Le patient amorce l'activité en allumant le four.

- Scénario 10 : Le patient met le lait après que les ingrédients sont mélangés.

- Scénario 11 : Le patient sort le plat du four avant de le faire cuire.

\section{- Jugement :}

- Scénario 12 : Le patient sort le plat du four sans mettre des gants.

- Scénario 13 : Le patient oublie d'éteindre le four.

\section{- Complétion :}

- Scénario 14 : Le patient mélange les ingrédients indéfiniment.

- Scénario 15 : Le patient ouvre et ferme la porte du four de façon répétitive.

- Scénario 16 : Le patient sort le gâteau du four mais ne le range pas.

- Scénario 17 : Le patient fait des actions inutiles après avoir sorti le gâteau du four. 


\section{- Entrecroisement :}

- Scénario 18 à 20 : Le patient effectue l'activité prendre un verre de lait en entrecroisement avec l'activité initiale confectionner un gâteau (entrecroisement de trois manières différentes).

Pour les fréquences d'observation des différents types de comportements erronés et cohérents, on établit un historique d'observation en s'inspirant des fréquences décrites dans l'étude de Giovannetti et al. [43], réalisée sur 51 patients réels souffrant de démences (maladies neurodégénératives).

\subsubsection{Présentation des résultats obtenus}

Les résultats exhibés à la Figure 4.6 montrent que l'agent de reconnaissance, en utilisant notre modèle, a été en mesure de prévoir, avant que le patient les réalise, presque $100 \%$ des comportements entrecroisés et des erreurs de type réalisation, et près de $70 \%$ des erreurs de type séquence. Ces résultats sont prometteurs, car toutes ces hypothèses reconnues n'étaient pas préétablies dans la base de connaissances; elles ont été dynamiquement générées dans l'espace de reconnaissance, en fonction d'un ensemble de plans possibles initialement identifié. Toutefois, notre approche est limitée, car on assume que la première action observée est correcte (pas d'erreurs) et cohérente avec le but du patient observé. Le problème est que, dans certains scénarios simulés, le patient exécute initialement une action qu'il doit, supposément, effectuer dans une étape ultérieure. Cette limitation explique les $30 \%$ des erreurs de séquence non prédites, et explique aussi pourquoi le système a de la difficulté à prévoir les erreurs d'initiation (0\%).

Les résultats montrent également que le modèle a été en mesure de prédire $50 \%$ des erreurs de type jugement et aucune erreur de type organisation. Parmi les erreurs de jugement et d'organisation, certaines d'entres-elles sont généralement difficiles à prévoir, parce que plutôt d'avoir comme origine un problème de planification, elles impliquent un problème de réalisation au niveau des actions de bas niveau. En d'autres mots, le patient réalise de façon incorrecte, ou non sécuritaire, une action d'une activité en cours d'exécution comme, par 


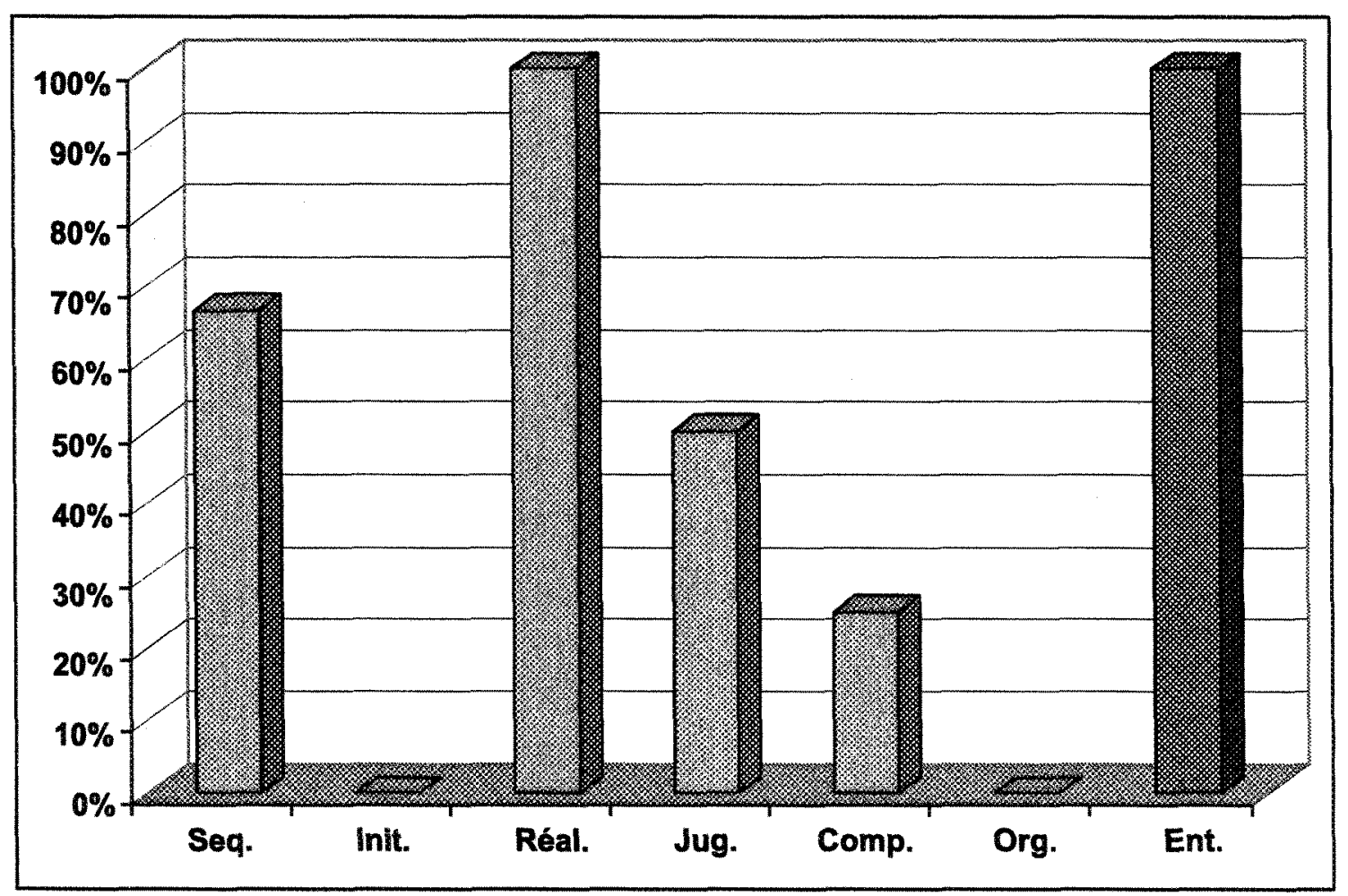

Figure 4.6 - Les différents plans reconnus par catégories

exemple, sortir du four un plat sans les gants ou mélanger un plat avec le mauvais ustensile. La gestion de ces erreurs de bas niveau serait plus facile en utilisant d'autres moyens, lesquels sont externes au modèle hybride de reconnaissance. Finalement, en ce qui concerne les erreurs de type complétion, le modèle a été seulement en mesure d'en prédire $25 \%$. La majorité des erreurs de ce type non prévues est reliée à des situations dans lesquelles le patient effectue de manière répétitive une ou des actions durant une période de temps indéterminée. Cet aspect de la problématique n'a pas été pris en compte dans le modèle initial et devra faire l'objet d'une réflexion plus approfondie dans nos futurs travaux de recherche.

La Figure 4.7 permet d'illustrer la comparaison proportionnelle de chacun des types de comportement erroné ou entrecroisé à travers l'ensemble d'activités anticipées. On remarque que les erreurs de type réalisation et de type séquence représentent $55 \%$ des comportements anticipés par le modèle. Ces deux catégories d'erreurs sont directement liées à des troubles de planification, lesquels sont caractéristiques des comportements erronés inhérents aux individus 
dont le niveau de dégénérescence de la maladie d'Alzheimer est dans les premiers stades $[68,69]$. Aussi, les comportements de type entrecroisement représentent $27 \%$ des comportements anticipés par le modèle. Ensemble, ces trois types de comportement représentent la majorité des types de comportement qu'un patient atteint de la maladie d'Alzheimer à un stade précoce à moyen effectue généralement. C'est pourquoi notre modèle porte une attention plus particulière à ces types de comportement. Conséquemment, on peut considérer que ces résultats sont prometteurs, car ils démontrent l'aptitude du modèle dans la prédiction des comportements typiquement effectués par les patients Alzheimer.

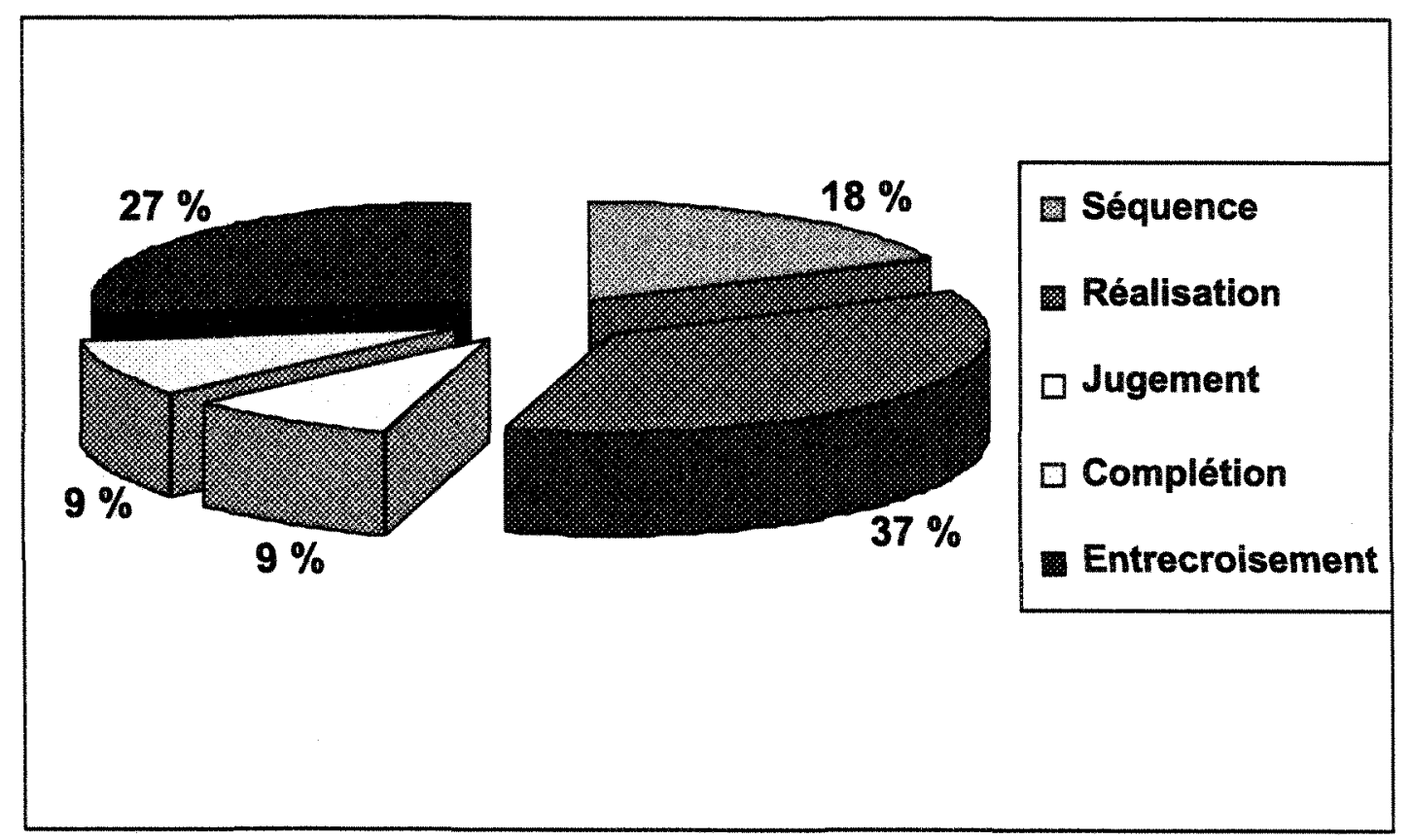

Figure 4.7 - Comparaison proportionnelle des types de plans anticipés

La Figure 4.8 permet d'illustrer l'efficacité du modèle pour filtrer les différentes hypothèses sur le comportement du patient observé. Le filtre logique permet de circonscrire l'ensemble des hypothèses pouvant être classifiées à l'intérieur de l'espace de reconnaissance. On note ici que le nombre totale d'hypothèses ne tient pas compte de la présence répétitive d'une action, pour ainsi obtenir une valeur fixe de comparaison. Le deuxième filtre, le filtre probabiliste, permet de réduire l'ensemble des hypothèses obtenu par le filtre logique en considérant seulement les hypothèses ayant les meilleures probabilités. Par conséquent, le filtre probabiliste permet 
de répondre à la problématique d'équiprobabilité des hypothèses soulevées par le modèle logique et permet de diminuer l'incertitude sur l'interprétation des actions observées. On peut remarquer la tendance générale que les hypothèses considérées par les deux filtres diminuent avec l'augmentation des actions observées. Par conséquent, plus l'épisode actuel du processus de reconnaissance est avancé, plus l'ensemble des hypothèses sur le comportement du patient est raffiné, pour ainsi augmenter la précision du processus d'assistance de l'agent assistant. Donc, plus qu'il y a d'actions observées par l'agent de reconnaissance, plus que l'efficacité des filtres logique et probabiliste augmente.

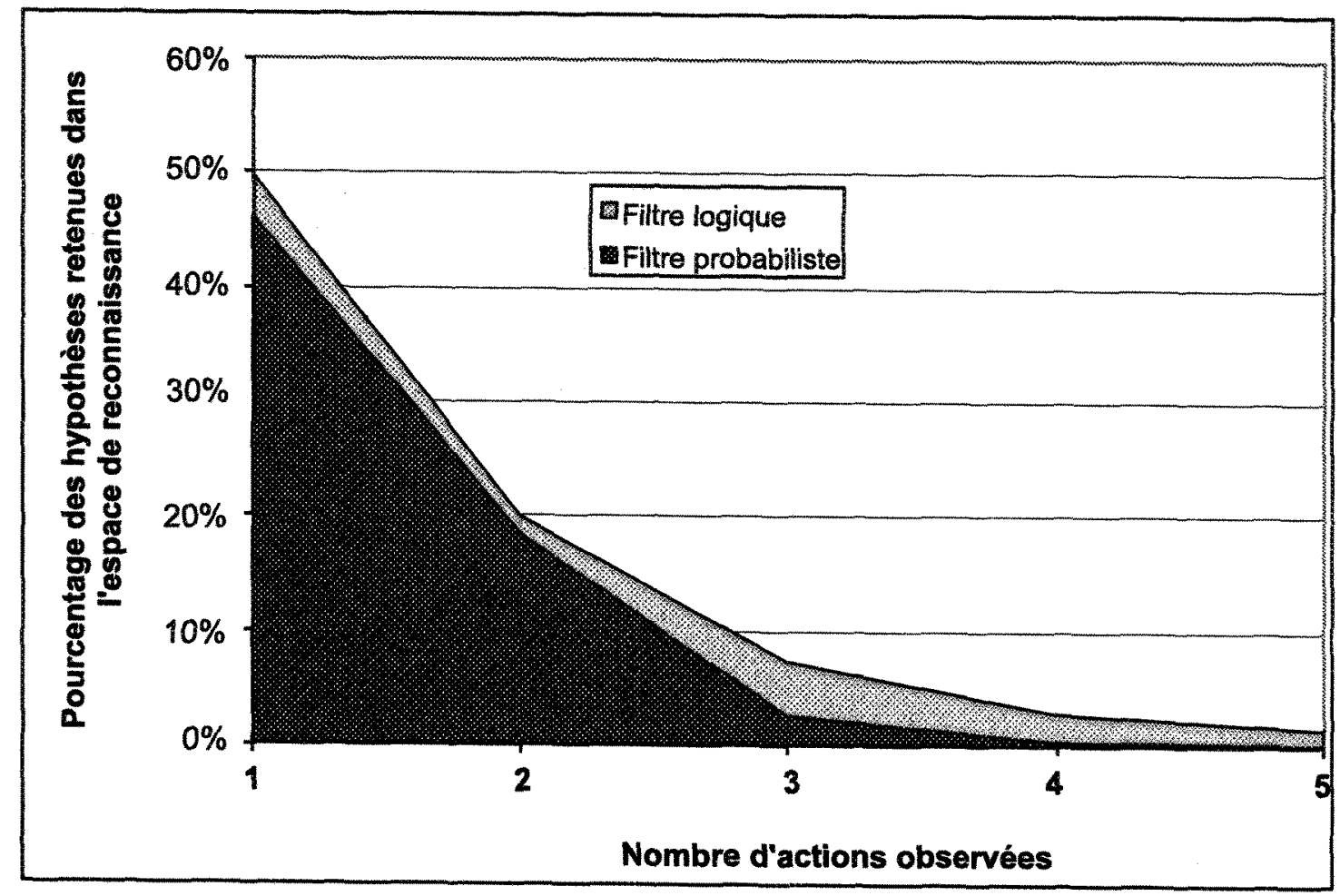

Figure 4.8 - Efficacité des filtres logique et probabiliste

Les résultats présentés à la Figure 4.9 illustrent l'évaluation empirique de la performance du système. On peut remarquer que le processus de reconnaissance de plans prend plus de temps dans la première phase (une moyenne de 658 millisecondes), lequel décroit de façon satisfaisante dans les phases subséquentes. La raison est que l'agent doit calculer, en utilisant les opérations de composition et de disunification, le schéma d'intentions qui synthétise les 
prédictions, et doit ensuite générer, en utilisant le processus de substitution des variables d'action, l'ensemble des nouvelles hypothèses caractérisant les plans erronés et entrecroisés possibles anticipés. En sachant que la base de connaissances contient des plans composés de 15 étapes en moyenne, ce résultat est prometteur. Dans un cas, on peut constater que le système prend plus de temps à calculer la deuxième observation que la première. Cela est expliqué par le fait que la deuxième observation amène plusieurs nouveaux plans possibles à partir de la librairie de plans. Chaque nouveau plan doit être inséré à l'intérieur du treillis en utilisant les opérateurs formels, ce qui prend un certain temps à calculer. Généralement, les ressources computationnelles requises pour la tâche de reconnaissance décroissent de manière significative après quelques observations, parce que seulement une faible quantité de nouveaux plans possibles sont identifiés.

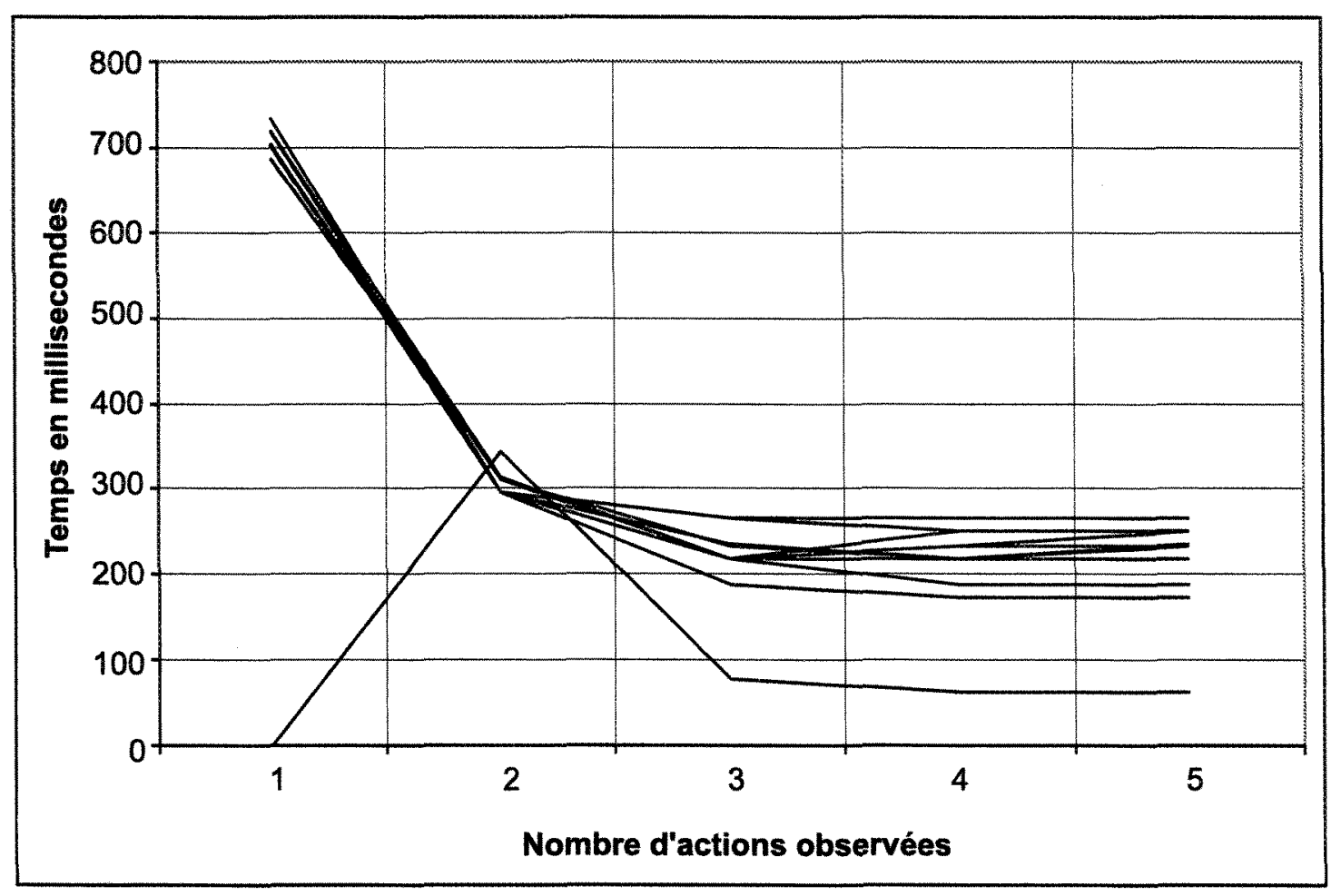

Figure 4.9 - Efficacité computationnelle du modèle de reconnaissance hybride en fonction du nombre d'observations. Sur ce graphique, chaque trait représente la simulation, action par action, d'un scénario de réalisation erronée ou entrecroisée différent et présente le temps de traitement requis pour chacune de ces actions 


\subsubsection{Discussions sur les résultats}

Malgré ces résultats prometteurs, un certain nombre de travaux subséquents seront requis pour pouvoir appliquer ce modèle dans un contexte à grande échelle. En premier lieu, pour évaluer de façon plus rigoureuse l'efficacité et les faiblesses du système, des expérimentations cliniques impliquant des patients et des thérapeutes seront nécessaires. Ces expérimentations permettront ainsi d'améliorer le système. Il existe plusieurs possibilités d'amélioration du modèle qui seront examinées lors de prochains travaux de recherche. L'une de ces possibilités est d'enrichir le modèle d'action pour tenir compte des différentes relations temporelles entre les actions qui composent un plan. En effet, la seule relation temporelle considérée présentement est que les actions doivent être exécutées selon une séquence particulière définie à l'intérieur de la structure de plan. Par exemple, un plan peut être exécuté de façon erronée si la durée acceptable entre deux étapes du plan est dépassée. Aussi, le patient peut effectuer deux actions en parallèle. Par conséquent, les aspects liés aux relations temporelles entre les actions pourront être pris en considération lors de l'élaboration des futures versions du modèle de reconnaissance. Finalement, on pourrait améliorer le modèle hybride en ajoutant un processus d'apprentissage, permettant de mieux établir les différentes probabilités associées aux plans. Par conséquent, on pourrait utiliser plus efficacement le profil de l'usager décrivant ses habitudes de vie.

\subsubsection{Comparaison avec les approches antérieures}

Il est, a priori, difficile de comparer objectivement nos résultats avec les résultats des travaux de Geib et al. [37] et des travaux de Avrahami et al. [4]. En effet, ces deux approches hybrides de reconnaissance de plans n'utilisent pas le même contexte pour effectuer les expérimentations. Les travaux de Geib et al. utilisent dans [37] un système de reconnaissance d'activités pour détecter les intrusions dans les systèmes de la part d'un agent hostile. À partir des différentes observations, le système de reconnaissance infère le but de l'agent hostile à partir des probabilités a posteriori calculées. Les travaux de Avrahami et al. utilisent dans [3] différentes configurations de la librairie de plans pour évaluer les performances empiriques du modèle. Du point de vue de la complexité, ces deux approches de reconnaissance 
de plans semblent plus efficaces que notre approche. Néanmoins, contrairement à notre approche, ces deux modèles ne génèrent pas dynamiquement de nouveaux plans plausibles (hypothèses), lesquels sont absents de la librairie de plans, expliquant le comportement erroné de l'entité observée. Les deux modèles proposés supposent que l'entité observée est rationnelle et, ainsi, ne tiennent pas compte de la problématique concernant le dilemme cité précédemment (erroné/entrecroisé).

Les travaux de Patterson et al. [70] et de Boger et al. [18] ont, quant-à eux, conduit des expérimentations similaires aux nôtres en utilisant des scénarios de cas réels sur un problème de taille comparable. Les travaux de Patterson et al. portent sur un système de reconnaissance des activités à l'intérieur d'un habitat intelligent à l'aide d'étiquettes RFID, laquelle est basée sur des modèles de Markov cachés [76] et sur un réseau bayésien dynamique [66]. Dans leurs expérimentations, le système de reconnaissance a été en mesure d'identifier l'activité de la vie quotidienne en cours d'exécution par la personne observée avec une précision d'environ $83 \%$ en moyenne, ce qui constitue un résultat impressionnant. De leur côté, les travaux de Boger et al. portent sur la reconnaissance d'une tâche impliquant le nettoyage des mains en utilisant un système de caméras, et sont basés sur les processus de décision de Markov partiellement observables [58]. Les expérimentations portaient sur la qualité des interventions du système d'assistance à la suite du processus de reconnaissance d'activités et présentent des résultats prometteurs. Il est difficile de comparer ces deux approches à notre modèle, car elles sont des approches purement probabilistes. En effet, le processus de reconnaissance de notre approche produit un ensemble d'hypothèses plausibles, lesquelles sont structurées à l'intérieur d'un espace de reconnaissance, qui ne sont pas, pour la plupart, prédéfinies dans la librairie de plan de l'agent observateur et correspondent aux comportements erronés ou entrecroisés possibles du patient. Le but de notre approche est d'anticiper les déviations comportementales pour permettre à un agent d'assistance de planifier une action d'assistance au patient. Néanmoins, malgré les résultats prometteurs, ces deux approches ne prennent pas en considération la problématique sur le dilemme (entrecroisé/erroné). 


\subsection{Conclusion}

Ce chapitre a permis de présenter le cas de validation ayant servis à concrétiser le modèle formel proposé au Chapitre 3. Dans la première partie du chapitre, les différents aspects de l'implémentation du modèle ont été présentés en montrant comment cette implémentation viendra s'intégrer à l'intérieur des infrastructures du laboratoire DOMUS. Aussi, l'architecture et le fonctionnement des différentes composantes de notre système de reconnaissance ont été présentés. Le système a été développé en ajoutant une couche probabiliste à l'application développée dans le cadre des travaux de thèse de Bruno Bouchard [21]. Cette application est une implémentation du modèle logique décrit dans les travaux de Bouchard et al. [22], et s'intègre à l'infrastructure du laboratoire DOMUS. Pour illustrer le fonctionnement du processus hybride de reconnaissance d'activités à l'intérieur de l'application développée, un scénario de reconnaissance a été présenté.

Dans la deuxième partie du chapitre, les détails concernant la phase de vérification du modèle hybride de reconnaissance réalisée à l'aide de l'application ont été présentés. La présentation des résultats obtenus lors de cette phase permet de montrer les forces et faiblesses du modèle hybride proposé, ainsi que certaines pistes d'amélioration future du modèle. Finalement, une comparaison entre les résultats de différentes approches antérieures et nos résultats ont été présentés à la fin de cette partie. 


\section{Chapitre 5}

\section{CONCLUSION GÉNÉRALE}

Malgré les progrès importants effectués dans le domaine de la reconnaissance de plans depuis les trente dernières années, le dilemme que nous avons soulevé dans ce travail de recherche est resté sans réponse, même s'il occupe une place significative à un niveau de base de la discipline et de ses applications. Ce mémoire propose une approche hybride pour s'attaquer à ce défi en exploitant la logique de description probabiliste. On doit souligner que ce cadre initial et nos résultats préliminaires n'amènent pas une solution exhaustive ou définitive aux multiples problèmes soulevés par la reconnaissance de plan. Toutefois, on peut considérer ce travail comme étant une première étape vers le développement d'une base théorique et uniforme pour caractériser le processus de reconnaissance de tout type de comportement, rationnel ou irrationnel. À ce titre, on note que notre approche a été implémentée et testée, montrant qu'elle peut fournir, à l'intérieur d'un habitat intelligent, une solution viable pour la reconnaissance du comportement d'un patient observé, en aidant le système à identifier ses opportunités d'assistance. On doit noter que l'originalité de notre approche est inhérente à l'étude algébrique de l'incertitude reliée au comportement d'un agent selon le point de vue computationnel. Une perspective intéressante pour l'enrichissement de ce modèle consiste à être en mesure de reconnaître des actions répétitives induites par le comportement du patient, et de prendre en compte les relations temporelles qui existent entre les différentes structures d'action.

D'un point de vue personnel, ce travail de recherche m'a permis de m'initier au processus de recherche scientifique. Plus spécifiquement, je me suis familiarisé au domaine de la 
reconnaissance de plans, à la logique de description, ainsi que la théorie des treillis. Durant les différentes étapes de réalisation du projet, j'ai eu l'occasion d'acquérir des connaissances qui me seront utiles durant ma future carrière. Cette expérience enrichissante m'a donné l'intérêt de poursuivre dans cette voie.

\subsection{Réalisation des objectifs fixés en introduction}

L'objectif général de ce travail de recherche était de définir un modèle de reconnaissance de plans pouvant reconnaître les comportements typiques d'un patient souffrant de la maladie d'Alzheimer à un stade léger à moyen (3-5), en explicitant formellement les plans erronés plausibles pouvant être induits par les symptômes de sa maladie. La reconnaissance des comportements futurs erronés permet à l'habitat intelligent de déterminer les différentes opportunités d'assistance à son occupant. Aussi, le modèle développé devait tenir compte du dilemme mentionné dans l'introduction : est-ce que l'observation d'une action différente à celle attendue peut s'interpréter comme étant la réalisation erronée d'une activité ou comme étant la réalisation entrecroisée de plusieurs activités? Cet objectif a été atteint à travers la réalisation de trois phases de recherche, chacune comportant des objectifs spécifiques.

La première phase du projet a permis d'effectuer une investigation en profondeur concernant notre problématique. Un premier volet a servi à effectuer une étude ciblée sur la maladie d'Alzheimer, afin de faciliter la compréhension des caractéristiques particulières du comportement des personnes atteintes. Ce volet a permis de mieux cerner les besoins inhérents à notre contexte applicatif spécifique, lié à la reconnaissance des activités de la vie quotidienne. Le second volet de cette phase a permis de faire une évaluation de différentes approches existantes $[17,27,55]$, plus particulièrement les approches hybrides $[4,38]$. Le résultat de cette étape, présenté au Chapitre 2, permet d'entrevoir différentes pistes de solutions à explorer en analysant les forces et faiblesses des différentes approches de reconnaissance. Entre autres, nous avons constaté que les différentes approches hybrides existantes ne tiennent pas compte du dilemme portant sur la différentiation du comportement entrecroisé et du comportement erroné. En somme, cette première phase nous a permis de mieux comprendre la problématique, 
d'évaluer différentes pistes de solutions possibles, de formuler un ensemble d'hypothèses de départ et de déterminer si celles-ci étaient fondées et pertinentes.

La deuxième phase du projet a permis, en utilisant les résultats des investigations précédentes, de mettre de l'avant nos hypothèses de recherche, dans le but de définir un modèle de reconnaissance de plans qui répond aux différents besoins identifiés lors de la première phase. L'approche adoptée consistait à améliorer le modèle logique décrit dans les travaux de Bouchard et al. [22], lequel est fondé sur un modèle d'action en logique de description et sur la théorie des treillis, en se basant sur la logique de description probabiliste. Cette étape a donc permis de définir les fondements théoriques pour un modèle de reconnaissance de plans hybride, lequel permet la reconnaissance et l'anticipation des comportements erronés et entrecroisés typiques des patients atteints de la maladie d'Alzheimer à un niveau léger à moyen.

La phase finale du projet a permis de valider ce modèle hybride, pour en vérifier sa viabilité et son opérationnalité au sein d'un contexte de simulation. À l'intérieur de cette phase, on a implémenté notre modèle hybride en ajoutant une couche probabiliste au système de reconnaissance d'activités développé par Bruno Bouchard lors de ses travaux de thèses à l'Université de Sherbrooke. Ce système de reconnaissance a permis d'implémenter le modèle logique qui était défini dans les travaux de Bouchard et al. [21,22], et qui s'intégrait à l'infrastructure du laboratoire DOMUS. La nouvelle couche du système nous a permis d'effectuer la gestion des probabilités utilisées par le processus hybride de reconnaissance d'activités pour générer l'espace de reconnaissance contenant les différentes hypothèses sur le comportement du patient observé. Un ensemble d'outils logiciels développés au laboratoire DOMUS, dont l'application de reconnaissance ACTIREC [12], ont servi pour implémenter le modèle et pour assurer qu'il pouvait s'intégrer au sein de l'architecture existante. Une vérification du système de reconnaissance développé a été effectuée en simulant un ensemble de scénarios de cas réels tirés de l'étude de Baum et al. [14,15], réalisée sur 106 patients Alzheimer, et en s'inspirant des fréquences d'observation fournies dans l'étude de Giovannetti et al. [43], réalisée sur 51 patients atteints de maladies neurodégénératives. À partir des résultats de cette phase de vérification, une évaluation des performances du système sur plusieurs aspects a 
permis de voir les différentes forces et faiblesses du modèle, ce qui nous a permis d'entrevoir différentes voies de développements futures pour l'amélioration du modèle.

\subsection{Apport du mémoire}

Notre contribution au domaine de la reconnaissance de plans et au domaine de l'assistance cognitive suit les traces des approches hybrides de reconnaissance de plans tout en essayant de répondre au dilemme cité en introduction. Elle correspond à la définition d'une structure de treillis, en utilisant une logique de description probabiliste [47], dans le but de formaliser un espace de reconnaissance composé d'hypothèses expliquant des comportements erronés et entrecroisés. Ce modèle de reconnaissance de plans [81] peut être vu comme étant une extension probabiliste d'un modèle logique présenté dans les travaux de Bouchard et al. [22].

L'utilisation de ce modèle logique, lequel est basé sur la théorie des treillis [29] et sur un cadre d'action formel [24] basé sur la logique de description [7], comme fondation de notre modèle hybride permet de transformer la problématique de la reconnaissance de plans en un problème de classification basé sur la relation de subsomption [42] provenant de la logique de description. Cette relation permet d'exploiter le paradigme de classification pour effectuer la correspondance entre un évènement observé et son type d'action qui lui correspond. Les plans possibles identifiés par ce processus de correspondance sont ensuite organisés à l'intérieur d'une structure de treillis, laquelle permet de rendre explicites les relations incertaines (partielles) entre ces plans possibles, lesquels ne sont pas forcément directement comparables. Par conséquent, on peut donc générer dynamiquement un ensemble d'hypothèses concernant les comportements erronés ou entrecroisés plausibles d'un patient souffrant de la maladie d'Alzheimer, lesquels peuvent être induits par son déficit cognitif.

Néanmoins, ce modèle logique souffre d'une problématique d'équiprobabilité des hypothèses plausibles. En fait, le modèle ne peut pas privilégier certaines hypothèses parmi l'ensemble des hypothèses générées par le processus logique. Pour pallier à cette limitation, le modèle hybride proposé consiste en une extension probabiliste de ce modèle logique. Cette extension est basée sur certains aspects de différents travaux portant sur les logiques de 
description probabilistes $[47,51,57]$. En effet, ce modèle hybride utilise la notion de relation de subsomption partielle $[47,51]$ entre les différents plans plausibles de l'espace de reconnaissance, laquelle utilise la notion d'intervalle de probabilités [19], pour désambiguïser l'ensemble des hypothèses du modèle d'interprétation (espace de reconnaissance) généré par un processus symbolique (le modèle logique). Par conséquent, l'utilisation des probabilités au sein du modèle hybride permet de privilégier certaines hypothèses et par conséquent, permet de répondre au problème d'équiprobabilité du modèle logique (le ccur du modèle hybride).

\subsection{Bilan du modèle de reconnaissance proposé}

Sommairement, notre approche hybride de reconnaissance [81] consiste à trouver un modèle d'interprétation minimal probabilisé correspondant à un ensemble d'actions observées, en construisant une structure de treillis de plans (espace de reconnaissance) à partir de l'ensemble des plans possibles. Dans un premier temps, un processus symbolique permet de minimiser l'incertitude qualitative reliée aux prédictions des actions futures du patient en générant dynamiquement un ensemble d'hypothèses sur son comportement à partir du schéma d'intentions. Ce schéma d'intentions correspond à la borne inférieure de la structure de treillis et est utilisé pour extraire un ensemble d'extra-plans erronés ou entrecroisés anticipés, lesquels ne sont pas prédéfinis dans la base de connaissances (librairie de plans) de l'agent et qui peuvent être potentiellement réalisés par le patient. Conséquemment, ce processus s'attaque au problème de complétion de la librairie de plans, laquelle est, dans un contexte réel, forcément incomplète peu importe le domaine. Ce processus symbolique permet de borner l'espace de reconnaissance en considérant seulement les hypothèses qui peuvent être classifiées à l'intérieur de la structure de treillis.

Dans un deuxième temps, un processus probabiliste utilise le modèle d'interprétation minimal généré par le processus symbolique et un historique de fréquences d'observation des réalisations erronées et cohérentes des activités pour minimiser l'incertitude reliée au problème d'équiprobabilité des hypothèses. En utilisant les fréquences de réalisation des différents plans de la librairie et des différents types de plans (erreurs et entrecroisement), le processus 
probabilise la structure de treillis en utilisant la relation de subsomption partielle. Ainsi, l'incertitude sur les actions futures du patient est minimisée, car on considère seulement les hypothèses ayant les meilleures probabilités. Donc, le processus hybride procure à l'agent d'assistance un moyen de décider le moment opportun où une action d'assistance doit être effectuée, en aidant l'habitat intelligent à identifier ses différentes opportunités d'assistance.

Lorsqu'on compare notre modèle avec les modèles hybrides de Avrahami et al. et Geib et al., on remarque que notre modèle semble a priori moins efficace du point de vue de la complexité. Ceci est causé par le fait que ces deux approches ne génèrent pas dynamiquement des hypothèses absentes de la librairie de plans. En d'autres mots, elles suggèrent, en fonction des observations, un ensemble de plans possibles existant dans la librairie de plans, lesquels peuvent être exécutés de manière entrecroisée. Par conséquent, ces deux approches ne prennent pas en considération le dilemme mentionné dans l'introduction, car elles considèrent que l'agent observé agit de façon rationnelle et, par conséquent, ne peut pas avoir un comportement incohérent. Donc, elles ne sont pas applicables dans un contexte d'assistance au patient Alzheimer au sein d'un habitat intelligent. Notre modèle, quant à lui, prend en considération le dilemme en générant dynamiquement des plans décrivant des comportements entrecroisés ou erronés de la part d'un patient. Ceci permet d'augmenter l'efficacité du processus de reconnaissance en augmentant sa précision dans la détection et la prédiction du comportement incohérent du patient. En améliorant le processus de reconnaissance, l'agent assistant est en mesure de mieux planifier ses différentes opportunités d'assistance au patient.

\subsubsection{Limitations de l'approche}

Le modèle de reconnaissance hybride proposé dans ce mémoire comporte certaines limitations, lesquelles sont identifiées lors des différentes étapes de validation. À partir des résultats obtenus à la suite de la vérification par simulation de scénarios de cas réels, même si on remarque que notre approche obtient de bons résultats dans la prévision des comportements de types réalisation, séquence, et entrecroisement, d'autres types de comportements sont plus difficilement anticipés. Cette limitation est principalement induite par certains postulats de 
départ trop rigide et par des aspects de la problématique qui n'ont pas été pris en considération initialement. À titre d'exemple, on ne tient pas compte du fait qu'un patient peut effectuer une action de façon répétitive ou bien amorcer la réalisation de l'activité par une action erronée.

Une autre limitation du modèle est que les structures de plans ne permettent pas de relations temporelles complexes. La seule relation temporelle considérée ici est que les actions doivent être organisées selon une séquence particulière. Dans un contexte réel, lorsqu'on planifie le plan pour accomplir une activité, on a la possibilité d'effectuer certaines actions avant ou après d'autres sans conséquence. Aussi, une autre limitation est que l'on considère seulement les actions contenues dans l'ensemble des plans possibles. Conséquemment, on ne peut pas prendre en compte le cas où le patient effectue une action étrangère, laquelle ne fait pas partie des plans possibles. On note également que le modèle est limité par la supposition que toutes les actions de bas niveau qui sont exécutables dans l'appartement sont présentes dans la base de connaissances de l'agent. Donc, il est possible que le patient réalise de nouveaux plans cohérents, non prédéfinis dans la librairie de plans, car ils contiennent au moins une action inconnue.

Pour ce qui est de la phase de vérification du modèle hybride proposé, nous avons une limitation sur le fait qu'on ne pouvions pas effectuer une expérimentation de l'implémentation dans un contexte réel impliquant des patients souffrant de la maladie d'Azheimer. En attendant la construction du centre de recherche en habitats intelligents à l'Université de Sherbrooke permettant d'effectuer des expérimentations sur des cas cliniques, nous avons utilisé des résultats provenant d'études sur des patients réels. Nous avons simulé un ensemble de scénarios de cas réels tiré de l'étude de Baum et al. $[14,15]$ en utilisant un historique d'observation inspiré des fréquences d'observation des différents types de comportements erronés et cohérents décrites dans l'étude de Giovannetti et al. [43]. Ces deux études ont été effectuées sur des patients réels souffrants de maladies neurodégénératives, dont fait partie la maladie d'Alzheimer. De plus, de prochains travaux de recherche permettront de recueillir une banque de données de résultats provenant de cas cliniques. 


\subsection{Perspectives de développements futurs}

Malgré ces résultats prometteurs, un certain nombre de travaux subséquents seront requis pour pouvoir appliquer ce modèle dans un contexte à grande échelle. En premier lieu, pour évaluer de façon plus rigoureuse l'efficacité et les faiblesses du système, des expérimentations cliniques impliquant des patients et des thérapeutes seront nécessaires. Ces expérimentations permettront ainsi d'améliorer le système en identifiant plus précisément les faiblesses du modèle. Il existe plusieurs possibilités possibles d'amélioration du modèle qui seront examinées lors de prochains travaux de recherche. L'une de ces possibilités est d'enrichir le modèle d'action pour tenir compte des différentes relations temporelles entre les actions qui composent un plan. En effet, la seule relation temporelle considérée présentement est que les actions doivent être exécutées selon une séquence particulière définie à l'intérieur de la structure de plan. Par exemple, un plan peut être exécuté de façon erronée si la durée acceptable entre deux étapes du plan est dépassée. Aussi, le patient peut effectuer deux actions en parallèle. Par conséquent, les aspects liés aux relations temporelles entre les actions pourront être pris en considération lors de l'élaboration des futures versions du modèle de reconnaissance. Finalement, on pourrait améliorer le modèle hybride en ajoutant un processus d'apprentissage, permettant de mieux établir les différentes probabilités associées aux plans. Par conséquent, on pourrait utiliser plus efficacement le profil de l'usager décrivant ses habitudes de vie pour établir les probabilités a. priori des activités de la vie quotidienne.

Pour conclure, je compte poursuivre des études au doctorat qui prendront en considération les aspects temporels pour améliorer le modèle de reconnaissance d'activités. Aussi, ces études permettront d'obtenir une banque de données sur des résultats de cas cliniques, permettant ainsi d'améliorer le processus de vérification du modèle. 


\section{BIBLIOGRAPHIE}

[1] Albrecht, D. W., Zukerman, I., And Nicholson, A. E. Bayesian models for keyhole plan recognition in an adventure game. User Modeling and User-Adapted Interaction 8 (1998), 5-47.

[2] Ankolekar, A., Burstein, M., Hobbs, J. R., Lassila, O., Martin, D. L., Mcilraith, S. A., Narayanan, S., Paolucci, M., Payne, T., Sycara, K., and Zeng, H. DAML-S : Semantic markup for web services. In Proceedings of the International Semantic Web Conference (ISWC) (2002), pp. 348-363.

[3] Avrahami-Zilberbrand, D., and Kaminka, G. A. Fast and complete symbolic plan recognition. In Proccedings of the IJCAI (2005).

[4] Avrahami-Zilberbrand, D., and Kaminka, G. A. Hybrid symbolic-probabilistic plan recognizer : Initial steps. In Proceedins of the AAAI Workshop on Modeling Others from Observations (2006).

[5] Avrahami-Zilberbrand, D., Kaminka, G. A., and Zarosim, H. Fast and complete symbolic plan recognition: Allowing for duration, interleaved execution, and lossy observations. In Proceedings of the IJCAI Workshop on Modeling Others from Observations (2005).

[6] Baader, F., Calvanese, D., McGuinness, D. L., Nardi, D., And Pater-Schneider, P. F., Eds. The Description Logic Handbook: Theory, Implementation and Applications. Cambridge University Press, 2003.

[7] BaAder, F., Horrocks, I., and Sattler, U. Description logics. In Handbook on Ontologies, S. Staab and R. Studer, Eds. Springer-Verlag, 2004.

[8] BaAder, F., Küsters, R., And Wolter, F. Extensions to description logics. In The Description Logic Handbook, F. Baader, D. Calvanese, D. McGuinness, D. Nardi, and P. Patel-Schneider, Eds. Cambridge University Press, 2003, pp. 219-261.

[9] BaAder, F., Lutz, C., Miličić, M., Sattler, U., And Wolter, F. Integrating description logics and action formalisms : First results. In Proceedings of the Twentieth National Conference on Artificial Intelligence (AAAI-05). (2005), pp. 572-577.

[10] BAAder, F., AND Nutt, W. Basic description logics. In The Description Logic Handbook, F. Baader, D. Calvanese, D. McGuinness, D. Nardi, and P. Patel-Schneider, Eds. Cambridge University Press, 2003, pp. 43-95. 
[11] Barwise, J., And Perry, J. Situations and attitudes. The Journal of Philosophy 78, 11 (1981), 668-691.

[12] BAUCHET, J. Une approche de la reconnaissance d'activité pour l'assistance dans les habitats intelligents. Master's thesis, Université de Sherbrooke, 2005.

[13] BAUChET, J., AND MAYERS, A. A modelisation of ADLs in its environment for cognitive assistance. In Proceedings of the 3rd International Conference on Smart Homes and Health Telematic (ICOST), Sherbrooke, Canada (2005), S. Giroux and H. Pigot, Eds., pp. 221-228.

[14] Baum, C., ANd Edwards, D. F. Cognitive performance in senile dementia of the Alzheimer's type : The kitchen task assessment. The American Journal of Occupationnal Therapy 47, 5 (1993), 431-436.

[15] Baum, C., Edwards, D. F., AND Morrow-Howell, N. Identification and measurement of productive behaviors in senile dementia of the Alzheimer type. The Gerontologist 33, 3 (1993), 403-408.

[16] BAYES, T. An essay towards solving a problem in the doctrine of chances. by the late Rev. Mr. Bayes, F. R. S. Communicated by Mr. Price, in a letter to John Canton, A. M. F. R. S. Philosophical Transactions (1683-1775) 53 (1763), 370-418.

[17] Boger, J., Hoey, J., Poupart, P., Boutilie, C., Fernie, G., and Mihailidis, A. A planning system based on Markov Decision Processes to guide people with dementia through activities of daily living. IEEE Transactions on Information Technology in BioMedicine 10, 2 (2006), 323-333.

[18] Boger, J., Poupart, P., Hoey, J., Boutilier, C., Fernie, G., And Mihailidis, A. A decision-theoretic approach to task assistance for persons with dementia. In Proceedings of the Nineteenth International Joint Conference on Artificial Intelligence (IJCAI) (2005), pp. 1293-1299.

[19] Boole, G. The Laws of Thought. Macmillan, London, 1854.

[20] Borgida, A. On the relative expressiveness of description logics and predicate logics. Artificial Intelligence 82 (1996), 353-367.

[21] BOUChARD, B. Un modèle de reconnaissance de plans pour les personnes atteintes de la maladie d'Alzheimer basé sur la théorie des treillis et sur un modèle d'action en logique de description. $\mathrm{PhD}$ thesis, Université de Sherbrooke, 2006.

[22] Bouchard, B., Bouzouane, A., And Giroux, S. A keyhole plan recognition model for Alzheimer's patients : First results. Applied Artificial Intelligence 22, 7 (August 2007), $623-658$.

[23] Bouzouane, A. Towards an authority sharing based on the description logic action model. In Proceedings of The 2005 IEEE/WIC/ACM International Conference on Intelligent Agent Technology (2005), pp. 277-280.

[24] Bouzouane, A., Bouchard, B., And Giroux, S. Action description logic for smart home agent recognition. In Proceedings of the International conference on HumanComputer Interaction (HCI) of the International Association of Science and Technology for Development (IASTED) (2005), pp. 185-190. 
[25] CARBerry, S. Techniques for plan recognition. User Modeling and User-Adapted Interaction 11 (2001), 31-48.

[26] Chalupsky, H., MacGregor, R. M., And Russ, T. PowerLoom Manual : Powerful knowledge representation and reasoning with delivery in Common-Lisp, Java, and $C++$. University of Southern California, Information Sciences Institute, 2006.

[27] Charniak, E., AND Goldman, R. G. A bayesian model of plan recognition. Artificial Intelligence 64 (1993), 53-79.

[28] Cohen, P. R., Perrault, C. R., and Allen, J. F. Beyond question answering. In Strategies for Natural Language Processing, W. G. Lehnert and M. H. Ringle, Eds. Lawrence Erlbaum Associates, 1982, pp. 245-274.

[29] Davey, B. A., AND Priestley, H. A. Introduction to Lattice and Order, second ed. Cambridge University Press, 2002.

[30] Diamond, J. A report on Alzheimer's disease and current research. Tech. rep., Alzheimer Society of Canada, 2005.

[31] Dix, J., Nanni, M., And Subrahmanian, V. S. Probabilistic agent programs. ACM Transactions on Computational Logic 1, 2 (2000), 208-246.

[32] Eiter, T., And GotTlob, G. The complexity of logic-based abduction. Journal of the Association for Computing Machinery 42, 1 (1995), 3-42.

[33] Eiter, T., Lu, J. J., Lukasiewicz, T., and Subrahmanian, V. S. Probabilistic object bases. ACM Transaction on Database Systems 26, 3 (2001), 264-312.

[34] ERol, K., Hendler, J., ANd NAU, D. S. HTN planning: Complexity and expressivity. In AAAI'94: Proceedings of the twelfth National Conference on Artificial Intelligence (1994), American Association for Artificial Intelligence, Ed., vol. 2.

[35] Fikes, R. E., AND Nilsson, N. J. STRIPS : A new approach to the application of theorem proving to problem solving. Artificial Intelligence 2 (1971), 189-208.

[36] Fine, S., Singer, Y., AND Tishby, N. The Hierarchical Hidden Markov model : Analysis and application. Machine Learning 32 (1998), 41-62.

[37] GeIB, C. W. Assessing the complexity of plan recognition. In Proceedings of AAAI (2004).

[38] Geib, C. W., And Goldman, R. P. Plan recognition in intrusion detection systems. In Proceedings of DARPA Information Survivability Conference 8 Exposition (DISCEX) II (2001).

[39] Geib, C. W., And Goldman, R. P. Partial observability and probabilistic plan/goal recognition. In Proceedings of the IJCAI workshop on Modeling Other from Observations (2005).

[40] Geib, C. W., AND Harp, S. A. Empirical analysis of a probabilistic task tracking algorithm. In Workshop on Agent Tracking, AAMAS (2004).

[41] Genesereth, M. R., And Nilsson, N. J. Logical Foundations of Artificial Intelligence. Morgan Kaufmann Publishers, 1987.

[42] GIL, Y. Description logics and planning. AI magazine 26(2) (2005), 73-84. 
[43] Giovannetti, T., Libon, D. J., Buxbaum, L. J., and Schwartz, M. F. Naturalistic action impairments in dementia. Neuropsychologia 40 (2002), 1220-1232.

[44] Goldman, R. P., Geib, C. W., and Miller, C. A. A new model of plan recognition. In Proceedings of the fifteenth Uncertainty in Artificial Intelligence Conference (1999).

[45] Grätzer, G. Two problems that shaped a century of lattice theory. Notices of the American Mathematical Society 54, 6 (2007), 696-707.

[46] Haton, J.-P., Bouzib, N., Charpillet, F., Haton, M.-C., LÂAsRi, B., LÂAsRi, H., Marquis, P., Mondot, T., AND NAPOLI, A. Le raisonnement en intelligence artificielle: Modèles, techniques et architectures pour les systèmes à bases de connaissances. InterEditions, 1991.

[47] Heinsohn, J. Probabilistic description logics. In Proceedings of the 10th Annual Conference on Uncertainty in Artificial Intelligence (1994), Morgan Kaufmann Publishers, pp. 311-318.

[48] Himsolt, M. GML : A portable graph file format (http://www.infosun.fim. uni-passau.de/Graphlet/GML/). Tech. rep., Université de Passau, Allemagne, 1996.

[49] Huet, G., And Oppen, D. C. Equations and rewrite rules : A survey. In Formal Language Theory : Perspectives and Open Problems, R. V. Book, Ed. Academic Press, 1980 , pp. 349-405.

[50] Isaacson, D. L., And Madsen, R. W. Markov Chains : Theory and Applications. John Wiley \& Sons, 1976.

[51] JAEger, M. Probabilistic reasoning in terminological logics. In Principles of Knowledge Representation and Reasoning : Proceedings of the Fourth International Conference (1994), J. Doyle, E. Sandewall, and P. Torasso, Eds., Morgan Kaufmann Publishers.

[52] Kaminka, G. A., And Avrahami, D. Symbolic behavior-recognition. In Proceedings of the AAMAS Workshop on Modeling Other Agents from Observations (2004).

[53] Kautz, H., And Allen, J. Generalized plan recognition. In Proceedings of the Fifth National Conference on Artificial Intelligence (1986), AAAI, Ed., pp. 32-37.

[54] KaUtz, H. A. A Formal Theory of Plan Recognition. PhD thesis, University of Rochester, 1987.

[55] KaUtz, H. A. A formal theory of plan recognition and its implementation. In Reasoning About Plans, J. F. Allen, H. A. Kautz, R. N. Pelavin, and J. D. Tenenberg, Eds. Morgan Kaufmann Publishers, 1991, ch. 2, pp. 69-126.

[56] KEMKE, C. A formal approach to describing action concepts in taxonomical knowledge bases. In Foundations of Intelligent Systems, N. Zhong, Z. W. Ras, S. Tsumoto, and E. Suzuki, Eds., Lecture Notes in Computer Science. Springer Berlin / Heidelberg, 2003, pp. $657-662$.

[57] Koller, D., Levy, A., And Pfeffer, A. P-ClASSIC : A tractable probabilistic description logic. In Proceedings of the Fourteenth National Conference on Artificial Intelligence (AAAI-97) (1997), pp. 390-397.

[58] LovejoY, W. S. A survey of algorithmic methods for partially observed Markov decision processes. Annals of Operations Research 28 (1991), 47-66. 
[59] MaCGregor, R. The evolving technology of classification-based knowledge representation systems. In Principles of Semantic Networks, J. F. Sowa, Ed. Morgan Kaufmann, 1991, pp. 385-400.

[60] MacGregor, R. A description classifier for the predicate calculus. In Proceedings of the twelfth National Conference on Artificial Intelligence (AAAI'94) (1994), American Association for Artificial Intelligence, Ed., pp. 213-220.

[61] Marconi, D. La Philosophie du Langage au Vingtième Siècle. Éditions De L'Éclat, 1997.

[62] MCCARTHY, J. Circumscription-a form of non-monotonic reasoning. Artificial Intelligence 13 (1980), 27-39.

[63] MCCARTHY, J. Applications of circumscription to formalizing common sense knowledge. Artificial Intelligence 28 (1986), 89-116.

[64] MCDERmotT, D. Estimated-regression planning for interactions with web services. In Proceedings of the Sixth International Conference on Artificial Intelligence Planning Systems (AAAI) (2002), pp. 204-211.

[65] MeNzEL, C. Actualism, ontological commitment, and possible world semantics. Synthese $85,3(1990), 355-389$.

[66] MurPhy, K. P. Dynamic Bayesian Networks : Representation, Inference and Learning. $\mathrm{PhD}$ thesis, University of California, Berkeley, 2002.

[67] Neapolitan, R. E. Learning Bayesian Networks. Prentice Hall, 2004.

[68] Patterson, D. J., Etzioni, O., Fox, D., and Kautz, H. Intelligent ubiquitous computing to support alzheimer's patients : Enabling the cognitively disabled. In Proceedings of UbiCog '02: First International Workshop on Ubiquitous Computing for Cognitive Aids, Göteborg, Sweden (2002), pp. 1-2.

[69] Patterson, D. J., Etzioni, O., and Kautz, H. The activity compass. In Proceedings of UbiCog '02: First International Workshop on Ubiquitous Computing for Cognitive Aids, Göteborg, Sweden (2002).

[70] Patterson, D. J., Fox, D., Kautz, H., And Philipose, M. Fine-grained activity recognition by aggregating abstract object usage. In Proceedings of IEEE 9th International Symposium on Wearable Computers (ISWC) (2005).

[71] Pearl, J., AND Russell, S. Bayesian networks. In Handbook of Brain Theory and Neural Networks, M. A. Arbib, Ed., second ed. MIT Press, 2002.

[72] Pigot, H., Mayers, A., And Giroux, S. The intelligent habitat and everyday life activity support. In Proceeding of the 5th International Conference in Simulations in Biomedicine, Slovenia (2003), pp. 507-516.

[73] Poole, D. Probabilistic Horn abduction and bayesian networks. Artificial Intelligence 64, 1 (1993), 81-129.

[74] Quinlan, J. R. C4.5 : Program for Machine Learning. Morgan Kaufmann Publishers, 1993.

[75] RABINER, L. R. A tutorial on Hidden Markov models and selected applications in speech recognition. In Proceedings of the IEEE (1989), vol. 77, pp. 257-286. 
[76] Rabiner, L. R., AND JUANG, B.-H. An introduction to Hidden Markov models. IEEE ASSP MAGAZINE 3, 1 (1986), 4-16.

[77] RAO, A. S. Means-end plan recognition : Towards a theory of reactive recognition. In Proceedings of the 4th International Conference on Principles of Knowledge Representation and Reasoning (1994), Morgan Kaufmann publishers, pp. 497-508.

[78] Reisberg, B. Stages of cognitive decline. The American Journal of Nursing 84, 2 (1984), $225-228$

[79] Reisberg, B., Ferris, S., Leon, M., and Crook, T. The global deterioration scale for assessment of primary degenerative dementia. AM J Psychiatry (1982), 1136-1139.

[80] ReITER, R. Knowledge in Action : Logical Foundations for Specifying and Implementing Dynamical Systems. The MIT Press, 2001.

[81] Roy, P., Bouchard, B., Bouzouane, A., And Giroux, S. A hybrid plan recognition model for Alzheimer's patients : interleaved-erroneous dilemma. In The 2007 IEEE / WIC / ACM International Conference on Intelligent Agent Technology (IAT 2007) (2007), pp. 131-137.

[82] Russell, S., And Norvig, P. Artificial Intelligence: A Modern Approach, second ed. Prentice Hall, 2003.

[83] Schmidt, C. F., Sridharan, N. S., and Goodson, J. L. The plan recognition problem : An intersection of psychology and artificial intelligence. Artificial Intelligence 11 (1978), 45-83.

[84] Sutron, R. S. On the significance of Markov decision processes. In Proceedings of the International Conference on Artificial Neural Networks (ICANN) (1997), pp. 273-282.

[85] Vergnes, D., Giroux, S., And Chamberland, D. Interactive assistant for activities of daily living. In Proceedings of the 3rd International Conference On Smart homes and health Telematics (ICOST), Sherbrooke, Canada (2005), pp. 229-236.

[86] Woвcke, W. Two logical theories of plan recognition. Journal of Logic Computation 12, 3 (2002), 371-412.

[87] Wooldridge, M. Reasoning about Rational Agents. The MIT Press, 2000. 